Singularidades analíticas reais e complexas 
SERVIÇO DE PÓS-GRADUAÇÃO DO ICMC-USP

Data de Depósito:

Assinatura:

\title{
Singularidades analíticas reais e complexas
}

\author{
Laís da Silva Oliveira
}

Orientador: Prof. Dr. Raimundo Nonato Araújo dos Santos

Dissertação apresentada ao Instituto de Ciências Matemáticas e de Computação - ICMC-USP, como parte dos requisitos para obtenção do título de Mestre em Ciências - Matemática . VERSÃO REVISADA 
Ficha catalográfica elaborada pela Biblioteca Prof. Achille Bassi e Seção Técnica de Informática, ICMC/USP, com os dados fornecidos pelo(a) autor(a)

d48s Silva Oliveira, Laís
Singularidades Analíticas Reais e Complexas / Laís
da Silva Oliveira; orientador Raimundo Nonato
Araújo dos Santos. -- São Carlos, 2013.
$85 \mathrm{p}$.
Dissertação (Mestrado - Programa de Pós-Graduação
em Matemática) - Instituto de Ciências Matemáticas
e de Computaçõo, Universidade de São Paulo, 2013.
1. fibração de Milnor local e global. 2.
topologia local e global da singularidade. 3.
fibraçóes de Milnor real e complexa. 4. trivialidade
topológica no infinito. 5. condições de regularidade
no infinito. I. Araújo dos Santos, Raimundo Nonato,
orient. II. Título.


Aos meus amados pais, Maria Nilda e Paulo Cesar. À minha saudosa avó, dona Rute. 
Lembra-te também do teu Criador nos dias da tua mocidade, antes que venham os maus dias, e cheguem os anos em que dirás: Não tenho prazer neles;

antes que se escureçam o sol e a luz, e a lua, e as estrelas, e tornem a vir as nuvens depois da chuva;

no dia em que tremerem os guardas da casa, e se curvarem os homens fortes, e cessarem os moedores, por já serem poucos, e se escurecerem os que olham pelas janelas,

e as portas da rua se fecharem; quando for baixo o ruído da moedura, e nos levantarmos à voz das aves, e todas as filhas da música ficarem abatidas;

como também quando temerem o que é alto, e houver espantos no caminho; e florescer a amendoeira, e o gafanhoto for um peso, e falhar o desejo; porque o homem se vai à sua casa eterna, e os pranteadores andarão rodeando pela praça;

antes que se rompa a cadeia de prata, ou se quebre o copo de ouro, ou se despedace o cântaro junto à fonte, ou se desfaça a roda junto à cisterna,

e o pó volte para a terra como o era, e o espírito volte a Deus que o deu.

Vaidade de vaidades, diz o pregador, tudo é vaidade.

Além de ser sábio, o pregador também ensinou ao povo o conhecimento, meditando, e estudando, e pondo em ordem muitos provérbios.

Procurou o pregador achar palavras agradáveis, e escreveu com acerto discursos plenos de verdade.

As palavras dos sábios são como aguilhões; e como pregos bem fixados são as palavras coligidas dos mestres, as quais foram dadas pelo único pastor.

Além disso, filho meu, sê avisado. De fazer muitos livros não há fim; e o muito estudar é enfado da carne.

Este é o fim do discurso; tudo já foi ouvido: Teme a Deus, e guarda os seus mandamentos; porque isto é todo o dever do homem. 
Porque Deus há de trazer a juízo toda obra, e até tudo o que está encoberto, quer seja bom, quer seja mau.

Eclesiastes 12 



\section{Agradecimentos}

Primeiramente agradeço a Deus, pelo dom da vida, e pela oportunidade de seguir meus sonhos e trabalhar no que gosto.

Agradeço à minha família, por sempre me apoiar mesmo quando eu "invento de ir pra tão longe" (fisicamente, mas sempre próximos no coração).

Agradeço aos amigos da UFRN, os que fizeram graduação comigo e os que tive o prazer de trabalhar junto no PET de Matemática. Ao meu amigo Marconio dos Santos, que nessa jornada se tornou um irmão.

Aos professores da UFRN, Marcelo Gomes, Ronaldo Freire, Viviane Campos, Roberto Hugo Bielschowsky e André Gustavo Pereira: pelos ensinamentos, tanto matemáticos como de vida. Agredeço muito pelo apoio aos estudos e por acreditarem em mim.

Agradeço aos meus amigos do ICMC, em especial a Ana Carolina Ramos, Apoenã Passamani, Ariadne Nogueira, Camilo Campana, Dione Lara, Evandro Nakajima, Steve Vicentin e Victor Barbosa, pelo grande apoio, quando tanto precisei, por cuidarem de mim e me fazerem sempre sorrir.

Agradeço muito ao meu amigo e orientador Raimundo Nonato, pelos grandes ensinamentos, pela paciência, preocupação e dedicação. Obrigada por acreditar em mim mais do que eu mesma! Muito obrigada!!!

Agradeço aos amigos Sheyla Marinho e Rawlilson Araújo, por terem me recebido muito bem em São Carlos e ajudado a orientar uma moça meio perdida sem conhecer nada nem ninguém.

A Jorge Deolindo e Camilo Campana, pela confecção das figuras neste trabalho. 
Aos professores do ICMC, que além dos conhecimentos matemáticos transmitidos, me serviram de exemplo de conduta profissional e, além do apoio, me deram a oportunidade de aprender com pesquisadores de excelência.

Aos funcionários da UFRN e do ICMC.

À Fapesp pelo apoio financeiro. 


\section{Resumo}

Neste projeto apresentamos algumas direções de pesquisa desenvolvidas no estudo da geometria/topologia da singularidade, no ambiente real e complexo, para funções e aplicações polinomiais. Para isso, utilizaremos as ferramentas da teoria de estratificação, técnicas de decomposição "Open book", condições de regularidade no sentido Malgrange, t-regularidade, $\rho_{E}$-regularidade e trivialidade topológica no infinito. 
Palavras-chave: valores atípicos; trivialidade topológica no infinito; fibração de Milnor local e global; topologia local e global da singularidade; fibrações de Milnor real e complexa; condições de regularidade no infinito. 


\section{Abstract}

On this project we present some research lines developed in the study of the geometry/topology of singularity, on the real and complex settings, for functions and polynomial mappings. For this, we use tools from stratification theory, techniques of "Open Book" decomposition, Malgrange regularity condition, $t$-regularity condition, $\rho_{E^{-}}$regularity and topological triviality at infinity.

Key words: atypical value; topological triviality at infinity; local and global Milnor's fibration; local and global topology of singularity; real and complex Milnor's fibrations; regularity conditions at infinity. 



\section{Sumário}

Introdução 1

1 Preliminares 5

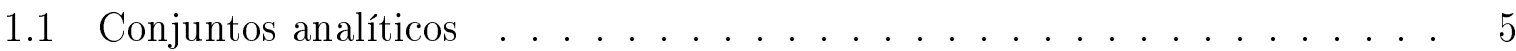

1.2 Germes de funções analíticas . . . . . . . . . . . . . . . 7

1.3 Noções básicas de Topologia Algébrica . . . . . . . . . . . . . . . . . . 9

1.3.1 Homotopia.......................... 9

1.3.2 Grupo fundamental . . . . . . . . . . . . . . . . . 12

1.3.3 Grupos de homotopia de ordem superior . . . . . . . . . . . . . 18

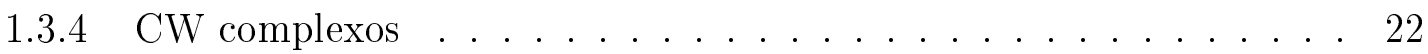

1.3.5 Suspensão e cone de um espaço topológico . . . . . . . . . . . . 27

1.3.6 Wedge de espaços topológicos . . . . . . . . . . . . . 29

1.3.7 Homologia Singular . . . . . . . . . . . . . . . . . 30

1.4 Noções básicas da Topologia Diferencial . . . . . . . . . . . . . . . 36

2 Singularidades de germes de funções holomorfas $\quad 41$

2.1 O teorema da fibração de Milnor . . . . . . . . . . . . . . . . 41

2.2 Topologia da fibra e do link . . . . . . . . . . . . . . . . 43

3 Aplicações holomorfas $\quad 51$

3.1 O link de uma Singularidade Isolada . . . . . . . . . . . . . . . 51

3.2 Teorema da fibração . . . . . . . . . . . . . . . . . 55 
4 Singularidades de aplicações reais $\quad 61$

4.1 As condições (a) e (b) de Milnor . . . . . . . . . . . . . . . 61

4.2 Condições de Milnor para funções holomorfas . . . . . . . . . . . . 65

4.3 Topologia da fibra de Milnor real . . . . . . . . . . . . . . 70

5 Singularidades no Infinito $\quad 73$

5.1 Estudo global da singularidade . . . . . . . . . . . . . 73

5.2 Fibração global . . . . . . . . . . . . . . . . . . 74 


\section{Introdução}

É do conhecimento geral que a existência da fibração de Milnor local (ou seja, em pequenas esferas em torno da singularidade) é a principal ferramenta no estudo da estrutura topológica da singularidade. Por outro lado, Henry King mostrou que uma condição necessária e suficiente para dois germes de aplicações analíticas serem $\mathcal{C}^{0}-\mathcal{A}$ equivalentes é que suas respectivas fibrações de Milnor sejam isomorfas. Portanto, a fibração de Milnor também desempenha um papel fundamental na classificação topológica dos germes analíticos.

Na última década, várias técnicas e ferramentas têm sido utilizadas no estudo da existência da fibração de Milnor real em esferas, para singularidades isoladas e não isoladas. Por exemplo, em [36] num trabalho conjunto de M. A. Ruas e R. Araújo dos Santos foi usada a teoria de estratificação com uma condição de regularidade, conhecida como "(C)regularidade de Karim Bekka", para garantir a existência da fibração de Milnor com a projeção dada pela projeção "canônica". Em [2], foi mostrado que é possível enfraquecer um pouco a condição acima usando uma condição de transversalidade chamada "Uniforme (m)-condition". E mais recentemente em [4] num trabalho conjunto de M. Tibar e R. Araújo dos Santos foi mostrada uma caracterização natural destes tipos de fibrações.

Ainda no aspecto local, em [24] D. Massey exibiu uma condição chamada de "Eanalytic" para garantir a existência da fibração de Milnor no chamado "tubo de Milnor". Em [13], T. Gaffney e R. Araújo dos Santos provaram que esta condição é muito restritiva para germes reais e mostraram como é possível utilizar a ferramenta de fecho integral 
real para módulos para garantir a existência da fibração numa família que não satisfaz a "E-analiticidade".

No aspecto global, ou seja, no estudo das singularidades no infinito, podemos verificar nos trabalhos de Némethi, Paunescu, Zaharia, Broughton, A. Bodin, M. Tibar e outros (ver p. exemplo [34, 30, 6, 47]) que a existência da fibração de Milnor no infinito (isto é, em esferas de raio suficientemente grande) também desempenha um papel importante no controle do comportamento das fibras no infinito. No entanto, o grande problema é que, diferentemente do caso local, não está claro que a fibração de Milnor no infinito sempre existe; exceto nos casos especiais de polinômios tame, quasitame, $M$-tame e semitame. Este fato, todavia, adiciona um ingrediente desafiador no estudo das singularidades no infinito.

Apresentaremos uma linha de estudos sobre a existência da fibração de Milnor local e global, nos casos real e complexo. Apresentaremos também, na medida do possível, resultados sobre a topologia da fibra e do link. Este trabalho foi desenvolvido da seguinte forma: no primeiro capítulo introduzimos conceitos e resultados básicos necessários para o desenvolvimento dos capítulos seguintes. No segundo capítulo, trabalhamos a existência da fibração de Milnor em esferas para funções complexas holomorfas e descrevemos os resultados feitos por J. Milnor sobre a topologia da fibra e do link. No terceiro capítulo, mostramos que a condição de ICIS para germes de aplicações complexas holomorfas é suficiente para garantir a existência da fibração de Milnor numa bola fechada e verificamos também que ainda neste caso a fibra de Milnor tem o tipo de homotopia de um buquê de esferas; em particular, tem também um alto grau de conexidade. No quarto capítulo, desenvolvemos um estudo sobre a existência da fibração de Milnor para germes de aplicações analíticas reais utilizando as condições de Milnor (a) e (b), como definidas por David Massey em [24]. Além disso, usamos a teoria de estratificação para mostrar que qualquer germe de função complexa holomorfa naturalmente satisfaz essas condições. Finalizamos esse capítulo apresentando um resultado de J. Milnor sobre o grau de conexidade da fibra real. No último capítulo (capítulo cinco), descrevemos algumas condições de regularidade que controlam o comportamento assintótico no infinito da fibra de uma função polinomial 
complexa. Apresentamos também um resultado devido a A. Bodin que mostra que no estudo de funções complexas planas a existência da fibração de Milnor no infinito (i.e., em grandes esferas) é equivalente à condição de todo valor regular não-nulo ser não atípico.

Estes estudos foram decorrentes do desenvolvimento do projeto de mestrado desenvolvido com o auxílio da Fapesp, processo número 2010/13321-1, sob o título "Singularidades analíticas reais e complexas". 


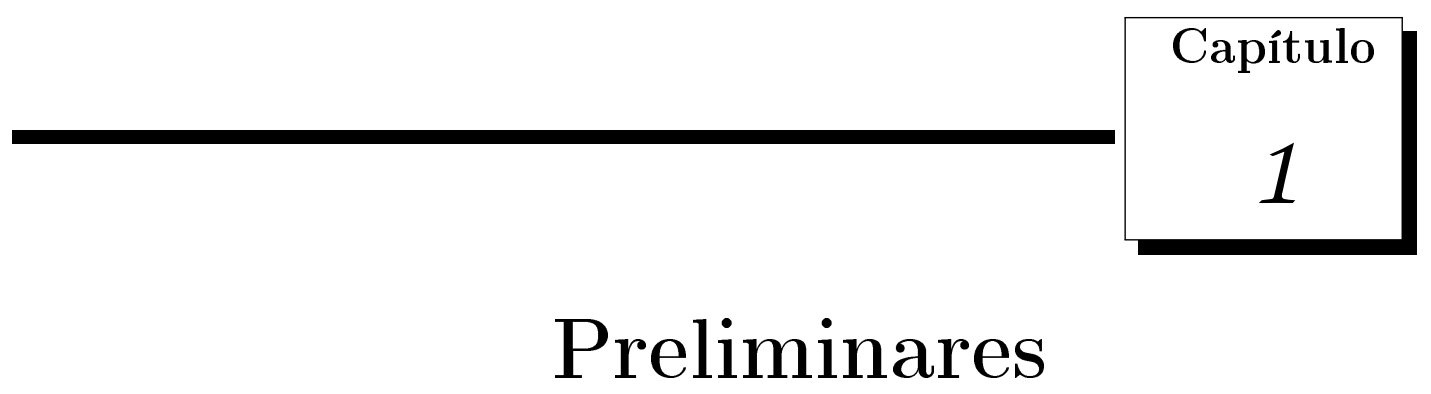

\subsection{Conjuntos analíticos}

Seja $\mathbb{K}$ o corpo dos números reais ou o corpo dos números complexos. Dado um conjunto aberto $\Omega$ em $\mathbb{K}^{n}$, dizemos que um conjunto $V \subset \Omega$ é analítico se para cada $a \in V$ existe uma vizinhança $U \subset \Omega$ e funções analíticas $f_{1}, \ldots, f_{p}$ definidas em $U$ tais que

$$
V \cap U=\left\{x \in U ; f_{1}(x)=\ldots=f_{p}(x)=0\right\}
$$

Observe que um conjunto analítico $V \subset \Omega$ é sempre fechado em $\Omega$.

Uma forma de analisar um conjunto analítico é usando ferramentas da topologia diferencial. Para isso, separamos o conjunto analítico de modo a aplicar estas ferramentas. Assim, introduzimos as seguintes definições.

Definição 1.1.1. Um ponto $x \in V$ é chamado de ponto regular de $V$ se existe uma vizinhança $U$ de $x$, com $U \subset \Omega$, tal que $V \cap U$ é uma subvariedade analítica de $U$, de dimensão digamos $d_{x}$. Um ponto $x \in V$ é chamado de ponto singular ou singularidade de $V$ se não for regular.

É facil ver que se um ponto é regular, então numa vizinhança do ponto todos os pontos são regulares. Ou seja, o conjunto dos pontos regulares de $V$ é aberto em $V$. 
O conjunto dos pontos singulares de $V$, denotado por $\Sigma(V)$, também é um conjunto analítico, chamado de conjunto singular de $V$. O conjunto $V \backslash \Sigma(V)$ é chamado de conjunto regular de $V$, ou de parte regular de $V$, e é denso em $V$.

Dada uma função analítica $f: \Omega \rightarrow \mathbb{K}$, considere o conjunto

$$
V(f)=f^{-1}(0)=\{x \in \Omega ; f(x)=0\} .
$$

Um conjunto analítico desta forma é chamado de hipersuperfície. Quando a hipersuperfície é definida por uma função analítica irredutível, o conjunto singular de $V(f)$ é dado pelos pontos que anulam o gradiente da função.

Dado um ponto $x \in \mathbb{K}^{n}$, considere a família $E_{x}$ de todos os conjuntos analíticos de $\mathbb{K}^{n}$ que contêm o ponto $x$. Definimos em $E_{x}$ uma relação de equivalência do seguinte modo: dois conjuntos $A, B \in E_{x}$ estão relacionados (notação: $A \approx_{x} B$ ) se existe uma vizinhança $V$ do ponto $x$ em $\mathbb{K}^{n}$ tal que $V \cap A=V \cap B$. As classes de equivalência são chamadas de germes de conjunto analítico no ponto $x$ e serão denotadas por $(X, x)$.

Diremos que um germe de conjunto analítico $X$ em $0 \in \mathbb{C}^{n}$ é irredutível, se toda vez que tivermos $X=Y \cup Z$, com $Y$ e $Z$ germes de conjuntos analíticos, temos que $X=Y$ ou $X=Z$. Seguindo [40] temos:

Definição 1.1.2. Seja $X$ um germe (não vazio) de conjunto analítico irredutível em $0 \in \mathbb{C}^{n}$. Seja $X_{0}$ a variedade (manifold) analitica, conexa, aberta e densa em X. Definimos e denotamos a dimensão de $X$ por

$$
\operatorname{dim}(X):=\operatorname{dim}\left(X_{0}\right)
$$

Dado X um germe analítico qualquer (não necessariamente irredutível), definimos a dimensão de $X$ como sendo a maior das dimensões de suas componentes irredutiveis. Se todas as componentes irredutiveis tem a mesma dimensão $k$, diremos que $X$ é de dimensão pura $k$.

Quando $\mathbb{K}=\mathbb{R}$, podemos estender naturalmente o conceito de conjunto analítico. Diremos que um conjunto $X \subset \mathbb{R}^{n}$ é semi-analítico se para cada $p \in X$ existe uma 
vizinhança aberta $U$ de $p$ em $\mathbb{R}^{n}$ e funções analíticas $f_{1}, \ldots, f_{k}, g_{1}, \ldots, g_{l}$ em $V$, tais que

$$
X \cap U=\left\{y \in U ; f_{1}(y)=\ldots=f_{k}(y)=0, g_{1}(y)>0, \ldots, g_{l}(y)>0\right\} .
$$

É claro que todo conjunto analítico é semi-analítico.

Neste contexto, temos uma ferramenta bastante útil, a saber o seguinte resultado.

Lema 1.1.1. (Lema de Seleção da Curva) Seja $X \subset \mathbb{R}^{n}$ um conjunto semi-analítico. Se $p \in \bar{X}$ (o fecho topológico de $X$ em $\mathbb{R}^{n}$ ), então existe uma curva real analítica $\gamma$ : $[0, \delta) \rightarrow X \cup\{p\} \operatorname{com} \gamma(0)=p$ e $\gamma(t) \in X$ para $t \in(0, \delta)$.

Uma prova deste resultado pode ser encontrada em [27] para conjuntos semi-algébricos e, com as devidas adaptações, pode ser estendida para conjuntos semi-analíticos.

\subsection{Germes de funções analíticas}

Seja $f: U \rightarrow \mathbb{K}^{p}$ uma aplicação analítica, definida em um aberto $U \subset \mathbb{K}^{n}, \operatorname{com} n \geq p$. Dizemos que $x \in U$ é um ponto regular de $f$ se o posto da matriz Jacobiana de $f$ em $x$ é máximo (ou seja, igual a $p$ ). Caso contrário, dizemos que $x$ é um ponto singular (ou uma singularidade) de $f$.

O conjunto $\Sigma(f):=\{x \in U ; x$ é ponto singular de $f\}$ é chamado de conjunto singular da aplicação $f$. Quando $p=1$, também chamamos $\Sigma(f)$ de conjunto crítico de $f$. Neste caso,

$$
\Sigma(f)=\{x \in U ; \nabla f(x)=0\}
$$

Denotamos por $\Delta(f)$ a imagem de $\Sigma(f)$ pela aplicação $f$, ou seja, $\Delta(f)=f(\Sigma(f))$, e chamamos de conjunto de valores críticos, ou conjunto discriminante de $f$.

Dado um ponto $x \in \mathbb{K}^{n}$, considere o conjunto $\mathcal{F}_{x, n}$ de todas as funções analíticas $f: U \rightarrow \mathbb{K}$ no ponto $x$, onde $U \subset \mathbb{K}^{n}$ é uma vizinhança de $x$. Definimos em $\mathcal{F}_{x, n}$ a seguinte relação: dados $f, g \in \mathcal{F}_{x, n}$, dizemos que $f: U_{1} \rightarrow \mathbb{K}$ e $g: U_{2} \rightarrow \mathbb{K}$ estão relacionadas, e denotamos $f \sim_{x} g$, se existe uma vizinhança $V$ de $x$, com $V \subset U_{1} \cap U_{2}$, tal que $\left.f\right|_{V}=\left.g\right|_{V}$. Esta relação é uma relação de equivalência em $\mathcal{F}_{x, n}$, e suas classes 
de equivalência são chamadas de germes de funções analíticas no ponto $x$. O conjunto quociente de $\mathcal{F}_{x, n}$ por esta relação é denotado por $\mathcal{O}_{x, n}$ quando $\mathbb{K}=\mathbb{C}$ e por $\mathcal{A}_{x, n}$ quando $\mathbb{K}=\mathbb{R}$. A classe da função $f$ é chamada então de germe de $f$ em $x$ e para representar esta classe denotamos $f:\left(\mathbb{K}^{n}, x\right) \rightarrow(\mathbb{K}, p)$, onde $p=f(x)$. No caso em que $x=0$, simplificamos a notação escrevendo $[f]_{0}$ ou $f:\left(\mathbb{K}^{n}, 0\right) \rightarrow(\mathbb{K}, f(0))$. Ainda neste caso, denotaremos o conjunto $\mathcal{O}_{0, n}$ (resp., $\left.\mathcal{A}_{0, n}\right)$ simplesmente por $\mathcal{O}_{n}$ (resp., $\mathcal{A}_{n}$ ).

Toda a construção a seguir pode ser feita $\operatorname{com} \mathcal{A}_{n}$ no lugar de $\mathcal{O}_{n}$.

Podemos introduzir em $\mathcal{O}_{n}$ uma estrutura de anel definindo as seguintes operações: dados $[f]_{0},[g]_{0} \in \mathcal{O}_{n}$,

$$
\begin{gathered}
{[f]_{0}+[g]_{0}:=[f+g]_{0},} \\
{[f]_{0} \cdot[g]_{0}:=[f \cdot g]_{0} .}
\end{gathered}
$$

É um exercício simples verificar que estas operações estão bem definidas, ou seja, independem da escolha dos representantes de cada classe. Com estas operações, $\mathcal{O}_{n}$ é um anel comutativo com unidade.

Considere agora o seguinte subconjunto de $\mathcal{O}_{n}$ :

$$
\mathcal{M}_{n}:=\left\{[f]_{0} ; f(0)=0\right\}
$$

Claro que $\mathcal{M}_{n} \neq \varnothing$, uma vez que $f \equiv 0 \in \mathcal{M}_{n}$. Além disso, $\mathcal{M}_{n}$ é um ideal de $\mathcal{O}_{n}$, pois para quaisquer $[f]_{0},[g]_{0} \in \mathcal{M}_{n}$ e $[h]_{0} \in \mathcal{O}_{n}$, temos

$$
[f]_{0}+[g]_{0} \in \mathcal{M}_{n} \text { e }[h]_{0} \cdot[f]_{0} \in \mathcal{M}_{n}
$$

Se $[g]_{0} \notin \mathcal{M}_{n}$, então $g(0) \neq 0$, logo existe uma vizinhança $U$ de 0 em $\mathbb{K}^{n}$ tal que $g(x) \neq 0$ para todo $x \in U$. Considere $h: U \rightarrow \mathbb{K}$ dada por $h(x)=\frac{1}{g(x)}$. Claro que $[h]_{0} \in \mathcal{O}_{n}$ e $[h]_{0} \cdot[g]_{0}=[1]_{0}$, logo $[g]_{0}$ é inversível. Com isto, concluímos que $\mathcal{M}_{n}$ é o único ideal maximal de $\mathcal{O}_{n}$.

Definição 1.2.1. Dizemos que um anel comutativo com unidade $R$ é um anel local se possui um único ideal maximal $\mathcal{M}$. Notação: $(R, \mathcal{M})$. 
Decorre do que vimos acima que $\left(\mathcal{O}_{n}, \mathcal{M}_{n}\right)$ e $\left(\mathcal{A}_{n}, \mathcal{M}_{n}\right)$ são anéis locais.

É possível provar também que $\mathcal{O}_{n}$ é um anel noetheriano; isto significa que todo ideal é finitamente gerado.

\subsection{Noções básicas de Topologia Algébrica}

Nesta seção reunimos conceitos e resultados da topologia algébrica que serão úteis no desenvolvimento do nosso estudo. As principais referências utilizadas são [20, 18].

\subsubsection{Homotopia}

Definição 1.3.1. Sejam $X, Y$ espaços topológicos. Duas aplicações continuas $f, g: X \rightarrow$ $Y$ dizem-se homotópicas quando existe uma aplicação contínua $H: X \times I \rightarrow Y$ tal que $H(x, 0)=f(x)$ e $H(x, 1)=g(x)$ para todo $x \in X$. A aplicação $H$ chama-se então uma homotopia entre $f$ e $g$. Escreve-se, neste caso, $H: f \simeq g$, ou simplesmente $f \simeq g$. Para cada $t \in I$, é interessante considerar também a aplicação $H_{t}: X \rightarrow Y$ dada por $H_{t}(x)=H(x, t)$. Com esta notação, $H_{0}=f$ e $H_{1}=g$.

Intuitivamente, uma homotopia pode ser pensada como uma deformação contínua da aplicação $f$ na aplicação $g$ ao longo do parâmetro $t$, que podemos pensar como sendo o tempo. Nessa abordagem, no tempo $t$ a aplicação $f$ é transformada na aplicação $H_{t}$.

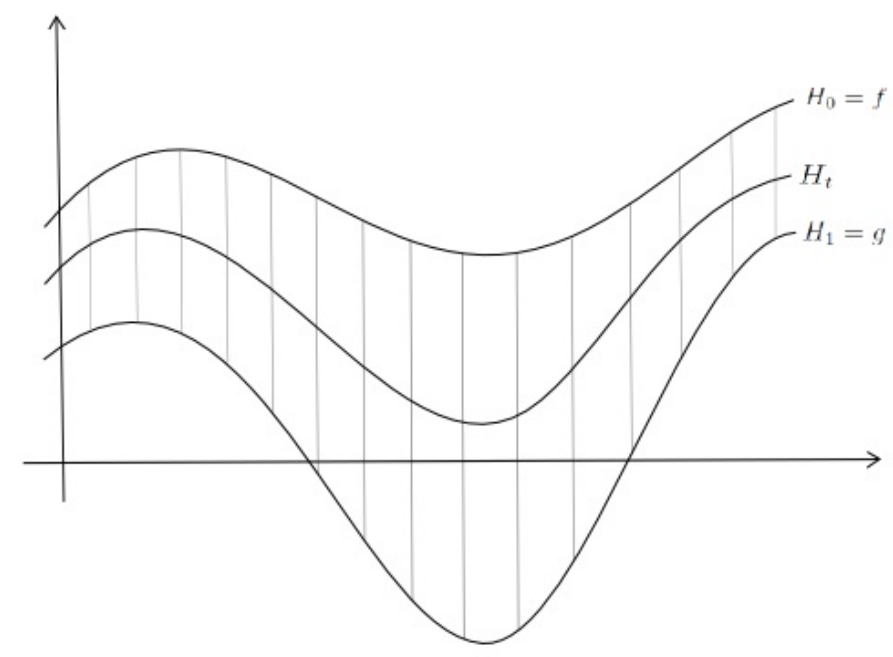


Exemplo 1.3.1. Seja $Y \subset \mathbb{R}^{n}$ um subconjunto convexo, isto é, para cada $x, y \in Y$, o segmento de reta fechado $[x, y]=\{(1-t) x+t y ; t \in[0,1]\}$ está contido em $Y$. Dado um espaço topológico $X$, quaisquer duas aplicações contínuas $f, g: X \rightarrow Y$ são homotópicas. De fato, a homotopia entre elas pode ser dada pela aplicação

$$
H(x, t)=(1-t) f(x)+t g(x),
$$

chamada de homotopia linear.

Proposição 1.3.1. Sejam $X, Y$ espaços topológicos. A relação de homotopia $f \simeq g$ é uma relação de equivalência no conjunto das aplicações contínuas de $X$ em $Y$.

As classes de equivalência são chamadas de classes de homotopia. A classe de homotopia de uma aplicação contínua $f: X \rightarrow Y$ é indicada pelo símbolo $[f]$. O conjunto das classes de homotopia das aplicações contínuas de $X$ em $Y$ é representado pelo símbolo $[X, Y]$.

Definição 1.3.2. Uma aplicação contínua $f: X \rightarrow Y$ chama-se uma equivalência homotópica quando existe $g: Y \rightarrow X$ continua tal que $g \circ f \simeq i d_{X}$ e $f \circ g \simeq i d_{Y}$. Diz-se então que g é uma inversa homotópica de $f$ e que os espaços $X$ e $Y$ têm o mesmo tipo de homotopia, ou que são homotopicamente equivalentes. Escrevemos, neste caso, $X \equiv Y$ ou $f: X \equiv Y$.

É claro que todo homeomorfismo é uma equivalência homotópica, mas a recíproca não é verdade em geral, conforme podemos perceber no seguinte exemplo. Ou seja, a classificação dos espaços módulo homotopia é bem mais ampla que a classificação módulo homeomorfismo.

Exemplo 1.3.2. Para todo $n \geq 1$ a esfera $S^{n}$ em $\mathbb{R}^{n+1}$ tem o tipo de homotopia de $\mathbb{R}^{n+1} \backslash\{0\}$. Considere as aplicações

$$
\begin{aligned}
& i: S^{n} \rightarrow \mathbb{R}^{n+1} \backslash\{0\} \quad r: \mathbb{R}^{n+1} \backslash\{0\} \quad \rightarrow \quad S^{n} \\
& x \mapsto x \quad y \mapsto \frac{y}{\|y\|}
\end{aligned}
$$


Então $r \circ i=i d_{S^{n}}$ e $i \circ r$ é homotópica à identidade de $\mathbb{R}^{n+1} \backslash\{0\}$ por uma homotopia linear, uma vez que cada ponto y de $\mathbb{R}^{n+1} \backslash\{0\}$ pode ser ligado a $\frac{y}{\|y\|}$ por um segmento de reta inteiramente contido em $\mathbb{R}^{n+1} \backslash\{0\}$.

Proposição 1.3.2. Sejam $f, f^{\prime}: X \rightarrow Y$ e $g, g^{\prime}: Y \rightarrow Z$ aplicações contínuas. Se $f \simeq f^{\prime}$ e $g \simeq g^{\prime}$ então $g \circ f \simeq g^{\prime} \circ f^{\prime}$.

É um exercício simples verificar que a relação de "mesmo tipo de homotopia" é uma relação de equivalência entre espaços topológicos.

Definição 1.3.3. Diz-se que um espaço topológico $X$ é contrátil quando ele tem o mesmo tipo de homotopia que um ponto.

Decorre da definição que um espaço $X$ é contrátil se, e somente se, a aplicação identidade $i d_{X}: X \rightarrow X$ é homotópica a uma aplicação constante $X \rightarrow X$. De fato, se $X$ é contrátil, então existe uma equivalência homotópica $f: X \rightarrow\{p\}$ para algum ponto $p \in X$. Segue desta afirmação que se um espaço $X$ é contrátil, então é conexo por caminhos.

Exemplo 1.3.3. Seja $Y \subset \mathbb{R}^{n}$ um subconjunto convexo. Então $Y$ é contrátil.

De fato, pelo Exemplo 1.3.1 quaisquer duas aplicações contínuas $f, g: X \rightarrow X$ são homotópicas, em particular se $f=i d_{X}$ e g é uma constante. Logo, concluímos que $Y$ é contrátil.

Exemplo 1.3.4. Se X é um espaço contrátil, então para qualquer espaço topológico $Y$, o produto cartesiano $X \times Y$ tem o mesmo tipo de homotopia que $Y$.

Seja $H$ uma homotopia entre $i d_{X}$ e uma aplicação constante $h: X \rightarrow X, h(x)=p$. Então

$$
\begin{aligned}
& f: X \times Y \rightarrow Y \quad e^{g: Y} \rightarrow X \times Y \\
& (x, y) \mapsto y \quad y \mapsto(p, y)
\end{aligned}
$$

são equivalências homotópicas, uma vez que $f \circ g=i d_{Y}$ e a aplicação

$$
\begin{aligned}
K: X \times Y \times I & \rightarrow X \times Y \\
(x, y, t) & \mapsto(H(x, t), y)
\end{aligned}
$$


define uma homotopia entre $i d_{X \times Y}$ e $g \circ f$.

Em particular, se $X$ e $Y$ forem contráteis, o produto cartesiano $X \times Y$ também é contrátil.

Definição 1.3.4. Seja $X$ um espaço topológico e $Y$ um subespaço de $X$. Uma aplicação continua $r: X \rightarrow Y$ chama-se uma retração quando se tem $r(y)=y$ para todo $y \in Y$, ou seja, quando $\left.r\right|_{Y}=i d_{Y}$.

Uma retração $r: X \rightarrow Y$ é, portanto, uma extensão contínua a $X$ da aplicação $i d_{Y}$. Note que toda retração é sobrejetiva. Quando existe uma retração $r: X \rightarrow Y$, o subespaço $Y$ chama-se um retrato do espaço $X$.

Decorre do Exemplo 1.3.2 que $S^{n}$ é um retrato de $\mathbb{R}^{n+1} \backslash\{0\}$.

\subsubsection{Grupo fundamental}

Diremos que $(X, A)$ é um par de espaços topológicos quando $A$ for um subespaço de $X$. Dados os pares $(X, A)$ e $(Y, B)$, uma aplicação contínua de pares

$$
f:(X, A) \rightarrow(Y, B)
$$

é uma aplicação contínua $f: X \rightarrow Y$ tal que $f(A) \subset B$.

Dadas as aplicações contínuas $f, g:(X, A) \rightarrow(Y, B)$, uma homotopia de pares entre $f$ e $g$ é uma aplicação contínua de pares

$$
H:(X \times I, A \times I) \rightarrow(Y, B)
$$

tal que $H(x, 0)=f(x)$ e $H(x, 1)=g(x)$ para todo $x \in X$. Decorre desta definição que $H_{t}(A) \subset B$ para todo $t \in I$.

Definição 1.3.5. Dadas $f, g: X \rightarrow Y$ contínuas, diz-se que $f$ é homotópica a $g$ relativamente a um subespaço $A \subset X$, e escreve-se

$$
f \simeq g(r e l . A)
$$


quando existe uma homotopia $H: f \simeq g$ tal que $H(x, t)=f(x)=g(x)$ para todo $x \in A$.

Exemplo 1.3.5. Considere $I=[0,1]$ e os caminhos continuos

$$
\begin{aligned}
& \alpha: I \rightarrow \mathbb{R}^{2} \\
& x \mapsto \alpha(x)=\left(x, 2 x^{2}-2 x+1\right) \quad{ }^{e} \quad x \mapsto \beta(x)=\left(x, 2 x^{3}-3 x^{2}+x+1\right)
\end{aligned}
$$

Observe que $\alpha(0)=\beta(0)$ e $\alpha(1)=\beta(1)$. Vimos no Exemplo 1.3 .1 que $\alpha$ e $\beta$ são homotópicos por uma homotopia linear

$$
H(x, t)=(1-t) \alpha(x)+t \beta(x)
$$

$e$, neste caso, $H(0, t)=\alpha(0)=\beta(0)$ e $H(1, t)=\alpha(1)=\beta(1)$ para todo $t \in I$. Logo,

$$
\alpha \simeq \beta(\text { rel. } \partial I)
$$

onde $\partial I=\{0,1\}$ é a fronteira de $I$.

Definição 1.3.6. Diremos que os caminhos continuos $\alpha, \beta: I \rightarrow X$ são caminhos homotópicos quando tivermos $\alpha \simeq \beta($ rel. $\partial I)$.

Notação: $\alpha \cong \beta$.

Assim, uma homotopia $H: \alpha \cong \beta$ entre caminhos é uma aplicação contínua $H$ : $I \times I \rightarrow X$ tal que

$$
\begin{gathered}
H(s, 0)=\alpha(s), H(s, 1)=\beta(s), \\
H(0, t)=\alpha(0)=\beta(0), \\
H(1, t)=\alpha(1)=\beta(1),
\end{gathered}
$$

quaisquer que sejam $s, t \in I$.

Em particular, se $\alpha, \beta: I \rightarrow X$ são caminhos fechados, isto é, $\alpha(0)=\alpha(1)$ e $\beta(0)=$ $\beta(1)$, então $\alpha \cong \beta$ quando existe uma aplicação contínua $H: I \times I \rightarrow X$ tal que, sendo $x_{0}=\alpha(0)=\alpha(1)$, tem-se

$$
H(s, 0)=\alpha(s), H(s, 1)=\beta(s), H(0, t)=H(1, t)=x_{0},
$$


para quaisquer $s, t \in I$.

Dados os caminhos $\alpha, \beta: I \rightarrow X$ com $\alpha(1)=\beta(0)$, considere os caminhos

$$
\begin{aligned}
& \alpha \beta: I \rightarrow X \\
& t \mapsto \begin{cases}\alpha(2 t) & \text { se } 0 \leq t \leq 1 / 2, \\
\beta(2 t-1) & \text { se } 1 / 2 \leq t \leq 1\end{cases} \\
& \alpha^{-1}: I \rightarrow X \\
& t \rightarrow \alpha(1-t)
\end{aligned}
$$

Tais caminhos $\alpha \beta$ e $\alpha^{-1}$ são chamados, respectivamente, de produto de $\alpha$ e $\beta$ e de caminho inverso de $\alpha$. É fácil ver que são contínuos. Além disso, com esta notação vale a seguinte propriedade:

Lema 1.3.1. Sejam $\alpha, \beta, \gamma: I \rightarrow X$ caminhos continuos tais que $\alpha(1)=\beta(0)$ e $\beta(1)=$ $\gamma(0)$. As seguintes afirmações são verdadeiras:

1. $\alpha \alpha^{-1} \cong \varepsilon_{x}$;

2. $\alpha^{-1} \alpha \cong \varepsilon_{y}$;

3. $\varepsilon_{x} \alpha \cong \alpha \cong \alpha \varepsilon_{y}$;

4. $(\alpha \beta) \gamma \simeq \alpha(\beta \gamma)$,

onde $x=\alpha(0), y=\alpha(1)$ e $\varepsilon_{x}, \varepsilon_{y}$ são os caminhos constantes $\varepsilon_{x}(s)=x, \varepsilon_{y}(s)=y$.

Lema 1.3.2. Se $\alpha \cong \alpha^{\prime}$ e $\beta \cong \beta^{\prime}$, então

$$
\alpha \beta \cong \alpha^{\prime} \beta^{\prime} \text { e } \alpha^{-1} \cong\left(\alpha^{\prime}\right)^{-1}
$$

Dado $X$ espaço topológico e $x_{0} \in X$, um caminho $\alpha: I \rightarrow X$ tal que $\alpha(0)=\alpha(1)=x_{0}$ é chamado um caminho fechado com ponto base $x_{0}$. Considere o conjunto

$$
\mathcal{C}_{x_{0}}:=\left\{\alpha: I \rightarrow X \text { caminho contínuo; } \alpha(0)=\alpha(1)=x_{0}\right\} .
$$


É fácil verificar que a relação $\cong$ é uma relação de equivalência em $\mathcal{C}_{x_{0}}$. Denote por $\pi_{1}\left(X, x_{0}\right)$ o espaço quociente de $\mathcal{C}_{x_{0}}$ pela relação de equivalência $\cong$. Denotaremos um elemento de $\pi_{1}\left(X, x_{0}\right)$ por $[\alpha]$, que representa a classe de todos os caminhos fechados com ponto base $x_{0}$ homotópicos a $\alpha$.

Com esta notação, definimos uma operação no conjunto $\pi_{1}\left(X, x_{0}\right)$ da seguinte forma:

$$
[\alpha] *[\beta]:=[\alpha \beta]
$$

Segue do Lema 1.3.2 que esta operação está bem definida. Pelo Lema 1.3.1 o elemento neutro desta operação é a classe $\left[\varepsilon_{x_{0}}\right]$ do caminho constante $\varepsilon_{x_{0}}(t)=x_{0}$. Além disso, $[\alpha]^{-1}=\left[\alpha^{-1}\right]$. Assim, esta operação define uma estrutura de grupo em $\pi_{1}\left(X, x_{0}\right)$, que chamaremos de grupo fundamental (ou primeiro grupo de homotopia) de $X$ com base em $x_{0}$.

Vale questionar a influência do ponto base na estrutura do grupo fundamental. Afim de sanar esta preocupação, geralmente são considerados espaços topológicos conexos por caminhos, e a seguinte proposição nos dá liberdade na escolha do ponto base.

Proposição 1.3.3. Se $X$ é um espaço topológico conexo por caminhos, então $\pi_{1}\left(X, x_{0}\right)$ é isomorfo a $\pi_{1}\left(X, \tilde{x}_{0}\right)$ para quaisquer $x_{0}, \tilde{x}_{0} \in X$.

Demonstração. Seja $\gamma: I \rightarrow X$ um caminho contínuo com $\gamma(0)=x_{0}$ e $\gamma(1)=\tilde{x}_{0}$. Definimos uma aplicação $\bar{\gamma}: \pi_{1}\left(X, x_{0}\right) \rightarrow \pi_{1}\left(X, \tilde{x}_{0}\right)$ por

$$
\bar{\gamma}([\alpha])=\left[(\gamma \alpha) \gamma^{-1}\right]
$$

Pelo Lema 1.3.2, esta aplicação está bem definida. De fato,

$$
\alpha \cong \beta \Rightarrow \gamma \alpha \cong \gamma \beta \Rightarrow(\gamma \alpha) \gamma^{-1} \cong(\gamma \beta) \gamma^{-1} \Rightarrow\left[(\gamma \alpha) \gamma^{-1}\right]=\left[(\gamma \beta) \gamma^{-1}\right]
$$

Por outro lado,

$$
\left((\gamma \alpha) \gamma^{-1}\right)\left((\gamma \beta) \gamma^{-1}\right) \cong(\gamma \alpha)\left(\gamma^{-1}\left(\gamma\left(\beta \gamma^{-1}\right)\right)\right) \cong(\gamma \alpha)\left(\left(\gamma^{-1} \gamma\right)\left(\beta \gamma^{-1}\right)\right)
$$


E também,

$$
\left(\gamma^{-1} \gamma\right)\left(\beta \gamma^{-1}\right) \cong \varepsilon_{\tilde{x}_{0}}\left(\beta \gamma^{-1}\right) \cong \beta \gamma^{-1}
$$

Segue disto e de (1.3.1) que

$$
\left((\gamma \alpha) \gamma^{-1}\right)\left((\gamma \beta) \gamma^{-1}\right) \cong(\gamma \alpha)\left(\beta \gamma^{-1}\right)
$$

Logo,

$$
\left((\gamma \alpha) \gamma^{-1}\right)\left((\gamma \beta) \gamma^{-1}\right) \cong(\gamma \alpha)\left(\beta \gamma^{-1}\right) \cong((\gamma \alpha) \beta) \gamma^{-1} \cong(\gamma(\alpha \beta)) \gamma^{-1}
$$

Ou seja, $\bar{\gamma}([\alpha]) * \bar{\gamma}([\beta])=\bar{\gamma}([\alpha] *[\beta])$. Temos assim que $\bar{\gamma}$ é um homomorfismo de grupos.

Do mesmo modo, temos que a aplicação

$$
\begin{aligned}
\overline{\gamma^{-1}}: \pi_{1}\left(X, \tilde{x}_{0}\right) & \rightarrow \pi_{1}\left(X, x_{0}\right) \\
{[\alpha] } & \mapsto\left[\left(\gamma^{-1} \alpha\right) \gamma\right]
\end{aligned}
$$

também é homomorfismo de grupos. Agora, segue dos lemas 1.3.1 e 1.3.2, de modo análogo ao que fizemos acima, que $\overline{\gamma^{-1}}$ é inversa de $\bar{\gamma}$, portanto $\bar{\gamma}$ é isomorfismo.

Nas condições da Proposição 1.3.3, denotamos o grupo fundamental $\pi_{1}\left(X, x_{0}\right)$ simplesmente por $\pi_{1}(X)$, uma vez que, a menos de isomorfismo, não depende da escolha do ponto base.

Notação: Se dois grupos $A, B$ são isomorfos, denotaremos este fato por $A=B$, sempre que não houver confusão. Faremos o mesmo quando nos referirmos a isomorfismo de estruturas algébricas, como espaços vetoriais, por exemplo.

Dados $X$ e $Y$ espaços topológicos e uma aplicação contínua de pares $f:\left(X, x_{0}\right) \rightarrow$ $\left(Y, y_{0}\right)$, então $f$ induz a seguinte aplicação

$$
\begin{aligned}
f_{*}: \pi_{1}\left(X, x_{0}\right) & \rightarrow \pi_{1}\left(Y, y_{0}\right) \\
{[\alpha] } & \mapsto[f \circ \alpha]
\end{aligned}
$$

Decorre das propriedade anteriores que $f_{*}$ é um homomorfismo de grupos. Além disso, dadas $f:\left(X, x_{0}\right) \rightarrow\left(Y, y_{0}\right)$ e $g:\left(Y, y_{0}\right) \rightarrow\left(Z, z_{0}\right)$ aplicações contínuas de pares, decorre 
da definição que

$$
(g \circ f)_{*}=g_{*} \circ f_{*} .
$$

E sendo $i d_{X}:\left(X, x_{0}\right) \rightarrow\left(X, x_{0}\right)$ a aplicação identidade, temos que $\left(i d_{X}\right)_{*}=i d_{\pi_{1}\left(X, x_{0}\right)}$.

Lema 1.3.3. Sejam $f, g: X \rightarrow Y$ aplicações contínuas homotópicas. Os homomorfismos

$$
f_{*}: \pi_{1}\left(X, x_{0}\right) \rightarrow \pi_{1}\left(Y, y_{0}\right) \text { e } g_{*}: \pi_{1}\left(X, x_{0}\right) \rightarrow \pi_{1}\left(Y, y_{1}\right)
$$

onde $y_{0}=f\left(x_{0}\right)$ e $y_{1}=g\left(x_{0}\right)$, são relacionados por $f_{*}=\bar{\gamma} \circ g_{*}$, onde $\bar{\gamma}: \pi_{1}\left(Y, y_{1}\right) \rightarrow$ $\pi_{1}\left(Y, y_{0}\right)$ é um isomorfismo definido por

$$
\bar{\gamma}([\alpha])=\left[(\gamma \alpha) \gamma^{-1}\right]
$$

e $\gamma: I \times X$ é um caminho contínuo tal que $\alpha(0)=y_{0}$ e $\alpha(1)=y_{1}$.

Proposição 1.3.4. Se dois espaços topológicos $X, Y$ são conexos por caminhos e têm o mesmo tipo de homotopia, então seus grupos fundamentais $\pi_{1}(X)$ e $\pi_{1}(Y)$ são isomorfos.

Para uma prova deste resultado é necessário apresentar vários outros resultados técnicos. Por este motivo, convidamos o leitor interessado a consultar o livro do Elon Lima $[20]$.

Corolário 1.3.1. Se $X$ e $Y$ são espaços topológicos conexos por caminhos e homeomorfos, então $\pi_{1}(X)=\pi_{1}(Y)$.

Corolário 1.3.2. O grupo fundamental de um espaço contrátil é trivial.

Definição 1.3.7. Um espaço topológico $X$ diz-se simplesmente conexo quando é conexo por caminhos e $\pi_{1}(X)=\{0\}$.

Exemplo 1.3.6. Do Exemplo 1.3.1 desta seção temos que o espaço $\mathbb{R}^{n}$ é contrátil e logo simplesmente conexo para todo $n \geq 1$. Vemos nos cursos básicos de Topologia que $S^{n}$ é conexo por caminhos para todo $n \geq 1$ e que $\pi_{1}\left(S^{1}\right)=\mathbb{Z}$. Além disso, não é difícil ver que para todo $n>1, \pi_{1}\left(S^{n}\right)=\{0\}$. Uma prova formal deste último fato pode ser encontrada em [20], página 44 . 
Uma aplicação simples, mas interessante do grupo fundamental é o seguinte resultado.

Proposição 1.3.5. (Teorema do ponto fixo de Brouwer) Seja $B^{2} \subset \mathbb{R}^{2}$ a bola fechada unitária e $f: B^{2} \rightarrow B^{2}$ uma aplicação contínua. Então, $f$ tem um ponto fixo, ou seja, existe $x \in B^{2}$ tal que $f(x)=x$.

Demonstração. Suponha que existe $f$ acima com $f(x) \neq x$ para todo $x \in D^{2}$. Para cada ponto $x$, considere a interseção do segmento de reta que liga $x$ a $f(x)$ com o bordo do disco $S^{1}$. Para determinar o ponto de interseção, considere a reta

$$
l(t)=x+t \frac{x-f(x)}{\|x-f(x)\|}
$$

Logo, basta determinar o único ponto $t_{0}$ que satisfaz a condição $l\left(t_{0}\right) \cap S^{1}$.

Claramente a construção acima determina uma função contínua $g: B^{2} \rightarrow S^{1}$ tal que $\left.g\right|_{S^{1}}=i d_{S^{1}}$, logo sobrejetora. Portanto, o funtor associado é um homomorfismo sobrejetor $g_{*}: \pi_{1}\left(B^{2}\right) \rightarrow \pi_{1}\left(S^{1}\right)$ o que é claramente impossível, visto que $\pi_{1}\left(B^{2}\right)=0$.

Uma outra propriedade importante do grupo fundamental é a seguinte.

Proposição 1.3.6. O grupo fundamental do produto cartesiano $X \times Y$ é isomorfo ao produto cartesiano dos grupos fundamentais de $X$ e $Y$ :

$$
\pi_{1}(X \times Y)=\pi_{1}(X) \times \pi_{1}(Y)
$$

Uma prova deste resultado pode ser encontrada em [20], página 45.

\subsubsection{Grupos de homotopia de ordem superior}

Podemos observar que a ideia intuitiva do grupo fundamental é a seguinte: dado um espaço topológico $X$ e um ponto base $x_{0} \in X$, podemos pensar um caminho fechado com ponto base $x_{0}$ como uma "rede unidimensional" no espaço, e as diferentes classes de homotopia como sendo as possíveis direções do espaço em que não podemos contrair o laço no ponto $x_{0}$, e que não são equivalentes umas com as outras. Ou seja, o grupo 
fundamental ser não trivial, significa que existe algum tipo de degeneração do espaço que é possível detectar com tais redes.

Embora o grupo fundamental forneça um invariante topológico interessante, ele não parece ser um invariante muito sensível a "pequenas degenerações" do espaço. Por exemplo, $\pi_{1}\left(\mathbb{R}^{3}\right)=\pi_{1}\left(\mathbb{R}^{3} \backslash\{0\}\right)$, mas claramente os espaços não são sequer homotópicos, uma vez que $\mathbb{R}^{3}$ é contrátil e $\mathbb{R}^{3} \backslash\{0\}$ tem a homotopia da esfera $S^{2}$. Além disso, $\pi_{1}\left(S^{2}\right)=\pi_{1}\left(\mathbb{R}^{2}\right)$ e esses espaços também não podem ser homeomorfos.

Afim de refinar esta distinção de espaços topológicos, são introduzidos os grupos de homotopia de ordem superior. Estendemos a noção de grupo fundamental de modo natural conforme o desenvolvimento a seguir.

Dado $n \in \mathbb{N}$, denotaremos por $I=[0,1], I^{n}=I \times \ldots \times I(n$ vezes $)$ e $\partial I^{n}$ o bordo do conjunto $I^{n}$. Observe que $\partial I^{n}$ é homeomorfo a $S^{n}$.

A definição a seguir é análoga à definição 1.3.5.

Definição 1.3.8. Dizemos que duas aplicações contínuas $\alpha, \beta: I^{n} \rightarrow X$, são homotópicas quando

$$
\alpha \simeq \beta\left(\text { rel. } \partial I^{n}\right)
$$

Denotamos este fato por $\alpha \cong \beta$.

Dadas as aplicações $\alpha, \beta: I^{n} \rightarrow X$, com $\alpha(1, x)=\beta(0, x)$ para todo $x \in I^{n-1}$, as aplicações

$$
\begin{gathered}
\alpha \beta(t, x)= \begin{cases}\alpha(2 t, x) & \text { se } 0 \leq t \leq 1 / 2, x \in I^{n-1} \\
\beta(2 t-1, x) & \text { se } 1 / 2 \leq t \leq 1, x \in I^{n-1}\end{cases} \\
\text { e } \\
\alpha^{-1}(t, x)=\alpha(1-t, x)
\end{gathered}
$$

são chamadas de produto de $\alpha$ e $\beta$ e inversa de $\alpha$, respectivamente.

Dado $x_{0} \in X$, uma aplicação de pares $f:\left(I^{n}, \partial I^{n}\right) \rightarrow\left(X, x_{0}\right)$ é chamada uma aplicação com ponto base $x_{0}$. No conjunto

$$
\mathcal{C}_{x_{0}}:=\left\{\alpha: I^{n} \rightarrow X \text { contínua; } \alpha\left(\partial I^{n}\right)=x_{0}\right\}
$$


a relação $\cong$ é uma relação de equivalência, cujo conjunto quociente $\mathcal{C}_{x_{0}} / \cong$ é denotado por $\pi_{n}\left(X, x_{0}\right)$ e a classe de equivalência da aplicação $\alpha \in \mathcal{C}_{x_{0}}$ é denotada por $[\alpha]$. Definimos em $\pi_{n}\left(X, x_{0}\right)$ a operação

$$
[\alpha] *[\beta]=[\alpha \beta]
$$

Esta operação fornece uma estrutura de grupo em $\pi_{n}\left(X, x_{0}\right)$.

Definição 1.3.9. O espaço $\pi_{n}\left(X, x_{0}\right)$ com a operação acima é chamado de n-ésimo grupo de homotopia do espaço $X$ com base em $x_{0}$.

Observe que quando $n=1$ recuperamos a definição que demos na seção anterior para o grupo fundamental $\pi_{1}\left(X, x_{0}\right)$. Além do mais, para $n=0$, podemos considerar $I^{0}$ como sendo um ponto e $\partial I^{0}$ o conjunto vazio. Daí, com as operações acima o grupo $\pi_{0}\left(X, x_{0}\right)$ representa as componentes conexas por caminhos de $X$ que contêm o ponto $x_{0}$. Assim, $\pi_{0}\left(X, x_{0}\right)=0$ significa que $X$ é conexo por caminhos.

Da mesma forma como ocorre com o grupo fundamental, para grupos de homotopia de ordem superior também valem os seguintes resultados.

Proposição 1.3.7. Se X é um espaço topológico conexo por caminhos, então para quaisquer $x_{0}, \tilde{x}_{0} \in X$ tem-se

$$
\pi_{n}\left(X, x_{0}\right)=\pi_{n}\left(X, \tilde{x}_{0}\right)
$$

para cada $n \in \mathbb{N}$.

Nas condições da Proposição 1.3.7, fixado $x_{0} \in X$, para cada $n \in \mathbb{N}$, denotamos o grupo de homotopia $\pi_{n}\left(X, x_{0}\right)$ simplesmente por $\pi_{n}(X)$, uma vez que, a menos de isomorfismo, o grupo não depende da escolha do ponto base $x_{0}$.

Análogo ao caso do grupo fundamental, dados $X$ e $Y$ espaços topológicos e $f$ : $\left(X, x_{0}\right) \rightarrow\left(Y, y_{0}\right)$ aplicação contínua de pares, temos para todo $n \in N$ uma aplicação induzida $f_{*}: \pi_{n}\left(X, x_{0}\right) \rightarrow \pi_{n}\left(Y, y_{0}\right)$ que é um homomorfismo de grupos. Além disso, dada $g:\left(Y, y_{0}\right) \rightarrow\left(Z, z_{0}\right)$ aplicação contínua, temos que a composta $g \circ f:\left(X, x_{0}\right) \rightarrow\left(Z, z_{0}\right)$ induz a seguinte igualdade $(g \circ f)_{*}=g_{*} \circ f_{*}$ e, sendo $i d_{X}:\left(X, x_{0}\right) \rightarrow\left(X, x_{0}\right)$ a aplicação identidade, temos que $\left(i d_{X}\right)_{*}=i d_{\pi_{n}\left(X, x_{0}\right)}$. Portanto, segue diretamente que se 
$f:\left(X, x_{0}\right) \rightarrow\left(Y, y_{0}\right)$ é um homeomorfismo, então $f_{*}: \pi_{n}\left(X, x_{0}\right) \rightarrow \pi_{n}\left(Y, y_{0}\right)$ é um isomorfismo de grupos, para todo $n \in \mathbb{N}$.

Proposição 1.3.8. Se $X$ e $Y$ são espaços topológicos conexos por caminhos e têm o mesmo tipo de homotopia, então

$$
\pi_{n}(X)=\pi_{n}(Y)
$$

para cada $n \in \mathbb{N}$.

Corolário 1.3.3. Se X é um espaço contrátil, então seu n-ésimo grupo de homotopia é trivial, para qualquer $n \in \mathbb{N}$.

Como extensão da Proposição 1.3.6, temos o seguinte resultado que, é claro, também vale para $n=1$.

Proposição 1.3.9. Dados $X$ e $Y$ espaços topológicos, conexos por caminhos, temos que $\pi_{n}(X \times Y)=\pi_{n}(X) \times \pi_{n}(Y)$, para todo $n \in \mathbb{N}$.

Decorre diretamente da proposição acima que para qualquer número finito de espaços topológicos, o resultado permanece verdadeiro. Além disso, é possível provar que o resultado também é verdadeiro para o produto cartesiano de uma família arbitrária de espaços topológicos. Uma prova deste resultado pode ser encontrada em [18], página 343, Proposição 4.2.

Em geral, para um espaço topológico $X, \pi_{1}(X)$ pode não ser abeliano. Para isso podemos usar, por exemplo, um espaço topológico que é homeomorfo à "figura 8". Para mais detalhes, ver [39], Exemplo 3.10, página 21.

Proposição 1.3.10. Para todo número natural $n \geq 2, \pi_{n}(X)$ é um grupo abeliano.

Demonstração. Ver [39], página 23, Teorema 3.1.2.

Definição 1.3.10. Dado $k \geq 0$ inteiro, dizemos que um espaço topológico $X$ é $k$-conexo se é conexo por caminhos e $\pi_{i}(X)=0$ para $0 \leq i \leq k$. 


\subsubsection{CW complexos}

Definição 1.3.11. Se $X$ e $Y$ são espaços topológicos, sua soma topológica (ou união disjunta) $X \sqcup Y$ é o conjunto $(X \times\{0\}) \cup(Y \times\{1\})$ munido da menor topologia que torna $X \times\{0\}$ e $Y \times\{1\}$ simultaneamente abertos e fechados e torna as inclusões

$$
\begin{array}{rlrl}
X & \rightarrow X \sqcup Y & Y & \rightarrow X \sqcup Y \\
x & \mapsto(x, 0) & y & \mapsto(y, 1)
\end{array}
$$

homeomorfismos sobre suas imagens.

Mais geralmente, se $\left\{X_{\alpha} ; \alpha \in A\right\}$ é uma familia indexada de espaços topológicos, então sua soma topológica $\bigsqcup_{\alpha \in A} X_{\alpha}$ é o conjunto $\bigcup_{\alpha \in A}\left(X_{\alpha} \times\{\alpha\}\right)$ munido da topologia que torna cada $X_{\alpha} \times\{\alpha\}$ simultaneamente aberto e fechado e que torna cada inclusão $x \mapsto(x, \beta)$ de $X_{\beta}$ em $\bigsqcup_{\alpha \in A} X_{\alpha}$ um homeomorfismo sobre sua imagem $X_{\beta} \times\{\beta\}$.

Definição 1.3.12. Sejam $X$ e $Y$ espaços topológicos e $A \subset X$ fechado. Seja $h: A \rightarrow Y$ uma aplicação contínua. Denotamos por $Y \cup_{h} X$ o espaço quociente da união disjunta $X \sqcup Y$ pela relação de equivalência $\sim$ definida da seguinte forma: para $u, v \in X \sqcup Y$, temos $u \sim v$ se, e somente se, pelo menos uma das seguintes condições é satisfeita:

1. $u=v$;

2. $u, v \in A$ e $h(u)=h(v)$;

3. $u \in A$ e $v=h(u) \in Y$.

$O$ espaço $Y \cup_{h} X$ é chamado de espaço colagem de $X$ a $Y$ via a aplicação $h$, e a aplicação $h$ é chamada de aplicação colagem.

Exemplo 1.3.7. Considere

$$
X=B^{2}, A=\partial B^{2}=S^{1}, Y=\{p\} .
$$

Então a aplicação colagem $h: A \rightarrow Y$ "colapsa" $A$ em um ponto, e o espaço $Y \cup_{h} X$ é homeomorfo à esfera $S^{2}$. 


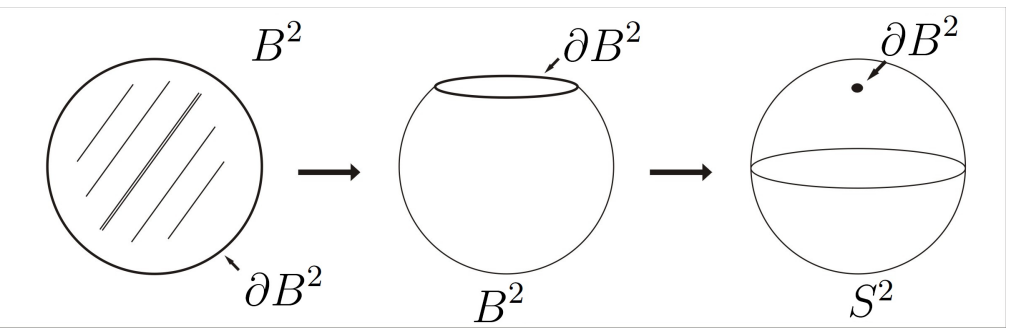

Nesta seção juntamos um número finito de espaços topológicos, cada um homeomorfo a uma $i$-bola aberta $B^{i} \backslash \partial B^{i}(0 \leq i \leq n)$, para formar um espaço topológico. Chamamos cada componente (homeomorfa a uma $i$-bola) de $i$-célula e a denotamos por $e^{i}$ (note que uma célula não tem bordo). Uma célula fechada $\bar{e}^{i}$ é homeomorfa à bola fechada $i$-dimensional. O bordo $\partial \bar{e}^{i}$ de $\bar{e}^{i}$ é homeomorfo a $S^{i-1}$. Assim, $\bar{e}^{i} \backslash \partial \bar{e}^{i}=e^{i}$. Adotamos a convenção que $\bar{e}^{0}=e^{0}$ é um ponto e que $\partial \bar{e}^{0}$ é vazio. Logo, as $0-$ células abertas são também 0-células fechadas.

Definição 1.3.13. Contruimos um espaço colagem chamado de $C W$ complexo (finito) da seguinte maneira: são dados

$$
\begin{gathered}
k_{0} \text { 0-células fechadas } \bar{e}_{1}^{0}, \bar{e}_{2}^{0}, \ldots, \bar{e}_{k_{0}}^{0}, \\
k_{1} 1 \text {-células fechadas } \bar{e}_{1}^{1}, \bar{e}_{2}^{1}, \ldots, \bar{e}_{k_{1}}^{1}, \\
\vdots \\
k_{n} n \text {-células fechadas } \bar{e}_{1}^{n}, \bar{e}_{2}^{n}, \ldots, \bar{e}_{k_{n}}^{n} .
\end{gathered}
$$

Nossa construção começa com $X^{0}:=\bar{e}_{0}^{0} \sqcup \bar{e}_{2}^{0} \sqcup \cdots \sqcup \bar{e}_{k_{0}}^{0}$, que é uma união disjunta. Tome $X^{(1)}=\bar{e}_{1}^{1} \sqcup \bar{e}_{2}^{1} \sqcup \cdots \sqcup \bar{e}_{k_{1}}^{1}$ (uma união disjunta) e $\partial X^{(1)}=\partial \bar{e}_{1}^{1} \sqcup \partial \bar{e}_{2}^{1} \sqcup \cdots \sqcup \partial \bar{e}_{k_{1}}^{1}$. Considere uma aplicação colagem

$$
h_{1}: \partial X^{(1)} \rightarrow X^{0}
$$

junte $X^{(1)}$ em $X^{0}$ por $h_{1}$ e obtenha o espaço colagem

$$
X^{1}=X^{0} \cup_{h_{1}} X^{(1)} .
$$


Tome $X^{(2)}=\bar{e}_{1}^{2} \sqcup \bar{e}_{2}^{2} \sqcup \cdots \sqcup \bar{e}_{k_{2}}^{2}$ e $\partial X^{(2)}=\partial \bar{e}_{1}^{2} \sqcup \partial \bar{e}_{2}^{2} \sqcup \cdots \sqcup \partial \bar{e}_{k_{2}}^{2}$. Considere novamente uma outra aplicação colagem

$$
h_{2}: \partial X^{(2)} \rightarrow X^{1}
$$

junte $X^{(2)}$ em $X^{1}$ por $h_{2}$ e obtenha o espaço colagem

$$
X^{2}=X^{1} \cup_{h_{2}} X^{(2)}
$$

Continuamos este processo até alcançar $X^{(n)}$. Faça

$$
X^{n}=X^{n-1} \cup_{h_{n}} X^{(n)} .
$$

O espaço final $X=X^{n}$ é um $C W$-complexo $n$-dimensional. Para cada $q, 0 \leq q \leq n, X^{q}$ é o q-esqueleto do $C W$-complexo $X$.

Seja $X$ um CW-complexo. Para cada $q$-célula $e_{j}^{q}$ temos a inclusão natural $i: \bar{e}_{j}^{q} \rightarrow$ $X^{(q)}$, a aplicação de identificação natural $\pi: X^{(q)} \rightarrow X^{q}=X^{q-1} \cup_{h_{q}} X^{(q)}$ e a aplicação de inclusão $\iota: X^{q} \rightarrow X$. A composta destas aplicações

$$
\phi_{j}^{q}=\iota \circ \pi \circ i: \bar{e}_{j}^{q} \rightarrow X
$$

é chamada a aplicação característica da célula $e_{j}^{q}$.

A restrição da aplicação característica $\phi_{j}^{q}$ ao bordo $\partial \bar{e}_{j}^{q}$ concorda com a restrição da aplicação colagem $h_{q}: \partial X^{(q)} \rightarrow X^{q-1}$ ao bordo $\partial \bar{e}_{j}^{q}$.

Observação 1.3.1. Pode ser que faltem células de alguma dimensão na nossa construção, mas o bordo $\partial X^{(q)}$ de $X^{(q)}=\bar{e}_{1}^{q} \sqcup \bar{e}_{2}^{q} \sqcup \cdots \sqcup \bar{e}_{k_{q}}^{q}$ deve sempre ser colado a algum subcomplexo $X^{r}$ por uma aplicação colagem

$$
h_{q}: \partial X^{(q)} \rightarrow X^{r}, r<q
$$

Teorema 1.3.1. Um $C W$-complexo $X$ é uma união disjunta finita de células abertas.

A partição de um CW-complexo $X$ em células abertas dada pelo Teorema acima é 
chamada de decomposição celular de $X$.

Definição 1.3.14. Dado $X$ um $C W$ complexo de dimensão $n$, denotaremos por $c_{j} o$ número de células de dimensão $j$ da decomposição celular de X. Neste caso, definiremos a característica de Euler de $X$ e denotaremos por

$$
\chi(X)=\sum_{i=0}^{n}(-1)^{i} c_{i}
$$

Observação 1.3.2. É possivel provar que a característica de Euler não depende da decomposição celular.

Exemplo 1.3.8. A esfera $n$-dimensional $S^{n}$ é um $C W$-complexo que consiste de uma 0-célula $\bar{e}^{0}$ e uma $n$-célula fechada $\bar{e}^{n}$ com aplicação colagem

$$
h_{n}: \partial \bar{e}^{n} \rightarrow \bar{e}^{0},
$$

isto é,

$$
S^{n}=\bar{e}^{0} \cup_{h_{n}} \bar{e}^{n}
$$

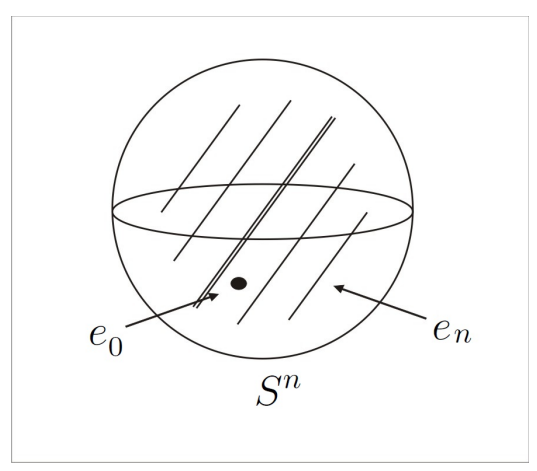

Neste caso, temos $c_{0}=1$ e $c_{n}=1$, portanto $\chi\left(S^{n}\right)=1+(-1)^{n}$.

Se colamos uma $n$-célula fechada $\bar{e}^{n}$ a $S^{n-1}=\bar{e}^{0} \cup_{h_{n-1}} \bar{e}^{n-1}$ pela aplicação identidade

$$
h_{n}: \partial X^{(n)}=S^{n-1} \rightarrow X^{n-1}=S^{n-1},
$$


o $C W$-complexo resultante é uma bola fechada n-dimensional

$$
B^{n}=\left(\bar{e}^{0} \cup_{h_{n-1}} \bar{e}^{n-1}\right) \cup_{h_{n}} \bar{e}^{n}
$$

Logo temos $c_{0}=1, c_{n-1}=1$ e $c_{n}=1$ e portanto, $\chi\left(B^{n}\right)=1+(-1)^{n-1}+(-1)^{n}=1$.

Exemplo 1.3.9. O toro $T^{2} \equiv S^{1} \times S^{1}$ consiste de uma 0 -célula fechada $\bar{e}^{0}$, duas 1 -células fechadas, $\bar{e}_{1}^{1}$ e $\bar{e}_{2}^{1}$, e uma 2-célula fechada $\bar{e}^{2}$ :

$$
S^{1} \times S^{1}=\left(\bar{e}^{0} \cup_{h_{1}}\left(\bar{e}_{1}^{2} \sqcup \bar{e}_{2}^{2}\right)\right) \cup_{h_{2}} \bar{e}^{2} .
$$

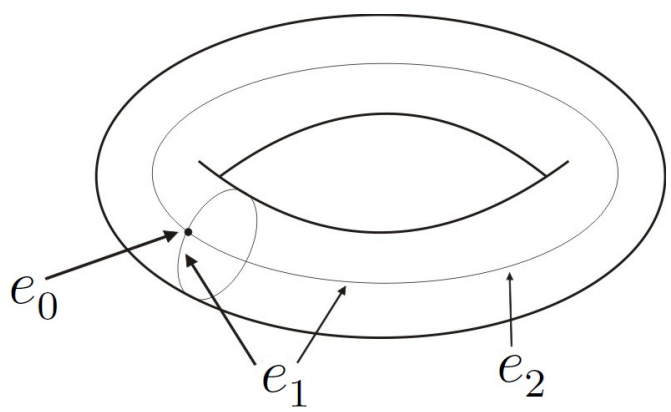

Logo, $\chi\left(S^{1} \times S^{1}\right)=1-2+1=0$.

Exemplo 1.3.10. O espaço projetivo real $P^{2}(\mathbb{R})$ pode ser visto como uma "colagem" de um disco fechado $B^{2}$ e uma Faixa de Moebius pelo bordo. Assim podemos enxergar a estrtutura de $C W$-complexo de $P^{2}(\mathbb{R})$ e sua decomposição celular:

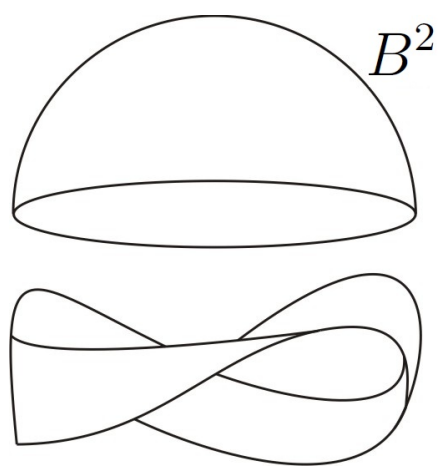

Logo, $\chi\left(P^{2}(\mathbb{R})\right)=1-2+2=1$. 
Proposição 1.3.11. O produto $X \times Y$ de dois $C W$-complexos $X$ e $Y$ é ainda um $C W$ complexo.

De fato, para cada célula $\bar{e}_{j}^{i}$ de $X$ e $\bar{e}_{l}^{\prime k}$ de $Y$ consideramos a célula $\bar{e}_{j}^{i} \times \bar{e}_{l}^{\prime k}$.

Proposição 1.3.12. Para um $C W$ complexo finito, conexidade e conexidade por caminhos são equivalentes.

Proposição 1.3.13. Quando $X$ é um $C W$ complexo, $\pi_{1}\left(X, x_{0}\right)$ é finitamente gerado, mas não necessariamente abeliano. Em particular, se $\pi_{1}\left(X, x_{0}\right)=0$, então para $n \geq 2$, $\pi_{n}\left(X, x_{0}\right)$ é um grupo abeliano finitamente gerado isomorfo a uma soma direta de algumas cópias de $\mathbb{Z}$ e algumas cópias de grupos cíclicos finitos $\mathbb{Z} /\left(p_{i}\right)$.

A importância do estudo da topologia do $C W$ complexo de dimensão finita vem do fato que toda variedade compacta de dimensão $n$ tem o tipo de homotopia de $C W$ complexo de dimensão $n$. Além disso, todo $C W$ complexo de dimensão $n$ é homotopicamente equivalente a uma variedade diferenciável $(2 n+1)$-dimensional.

\subsubsection{Suspensão e cone de um espaço topológico}

Dado um espaço topológico $X$, a suspensão $\mathcal{S}(X)$ de $X$ é o espaço quociente obtido a partir de $X \times I$, colapsando $X \times\{0\}$ em um ponto e $X \times\{1\}$ em outro ponto. Por exemplo, quando $X$ é a esfera 1 -dimensional $S^{1}$ (vamos olhá-la mergulhada em $\mathbb{R}^{3}$ ), primeiro observamos o conjunto $X \times I$, que é um pedaço de cilindro. Daí, colapsamos os conjuntos $S^{1} \times\{0\}=\left\{(x, y, 0) \in \mathbb{R}^{3} ; x^{2}+y^{2}=1\right\}$ e $S^{1} \times\{1\}=\left\{(x, y, 1) \in \mathbb{R}^{3} ; x^{2}+y^{2}=1\right\}$ nos pontos, digamos, $(0,0,0)$ e $(0,0,1)$. Temos então um espaço homeomorfo à esfera $S^{2}$. De modo geral, podemos estender este raciocínio quando $X$ é a esfera $n$-dimensional $S^{n}$, e neste caso $\mathcal{S}(X)$ é (homeomorfo a) $S^{n+1}$.

Quando $X$ é um CW-complexo, $\mathcal{S}(X)$ também é, como quociente do CW-complexo $X \times I$ colapsando os subcomplexos $X \times\{0\}$ e $X \times\{1\}$ em duas 0-células.

A suspensão é invariante por tipo de homotopia, no seguinte sentido: se $X$ e $Y$ têm o mesmo tipo de homotopia, então $\mathcal{S}(X)$ e $\mathcal{S}(Y)$ têm o mesmo tipo de homotopia. Conse- 


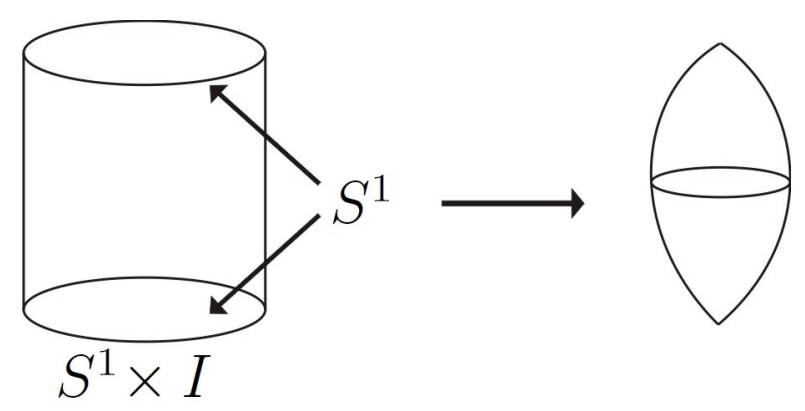

quentemente, se $X$ e $Y$ são conexos por caminhos, temos

$$
\pi_{n}(\mathcal{S}(X))=\pi_{n}(\mathcal{S}(Y))
$$

com $n$ inteiro positivo fixo.

O cone sobre $X$, denotado por $\mathcal{C}(X)$, é obtido a partir de $X \times I$, colapsando $X \times\{0\}$ em um ponto. Quando $X=S^{n}$, o cone sobre $X$ é um espaço homeomorfo à bola fechada $B^{n+1}$. Outra forma de interpretar o cone sobre um espaço é como a união de todos os segmentos de reta que ligam pontos de $X$ com um ponto $p$ fixo. Assim como ocorre com a

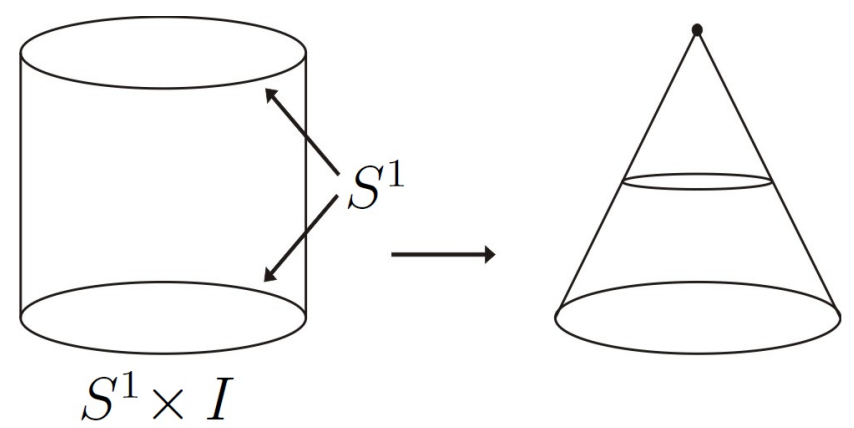

suspensão, quando $X$ é um $C W$-complexo temos que $\mathcal{C}(X)$ também é um $C W$-complexo. No caso da estrutura de cone, o tipo de homotopia não é preservado, como podemos observar quando $X=S^{1}$ :

$$
\pi_{1}\left(S^{1}\right)=\mathbb{Z}, \quad \text { e } \pi_{1}\left(\mathcal{C}\left(S^{1}\right)\right)=\pi_{1}\left(B^{2}\right)=0
$$




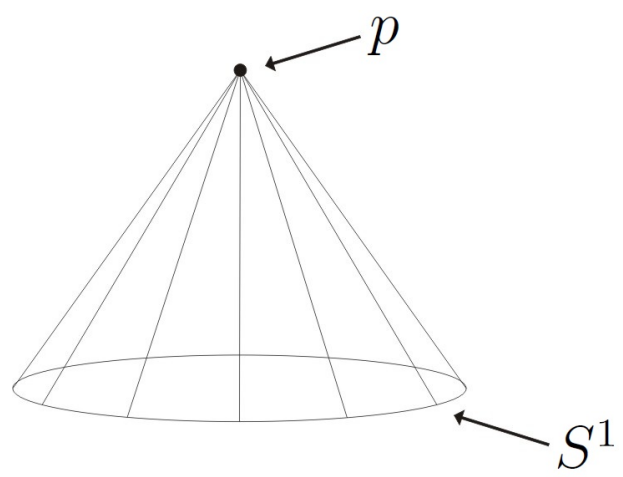

\subsubsection{Wedge de espaços topológicos}

Sejam $X$ e $Y$ espaços topológicos. Dados os pontos $x_{0} \in X$ e $y_{0} \in Y$, obtemos um novo espaço topológico, chamado de wedge de $X$ e $Y$, a partir da união disjunta $X \sqcup Y$ identificando $x_{0}$ e $y_{0}$ em um único ponto. Denotamos o wedge de $X$ e $Y$ por $X \vee Y$.

Naturalmente, podemos estender esta noção para uma família arbitrária de espaços topológicos $X_{\alpha}$, fixando, para cada índice $\alpha$, um ponto $x_{\alpha} \in X_{\alpha}$ e partindo da união disjunta $\bigsqcup_{\alpha} X_{\alpha}$ identificamos os pontos $x_{\alpha}$ em um único ponto.

Exemplo 1.3.11. Se $X=Y=S^{1}$, o espaço $S^{1} \vee S^{1}$ é homeomorfo à figura 8, dois círculos ligados por um único ponto comum.

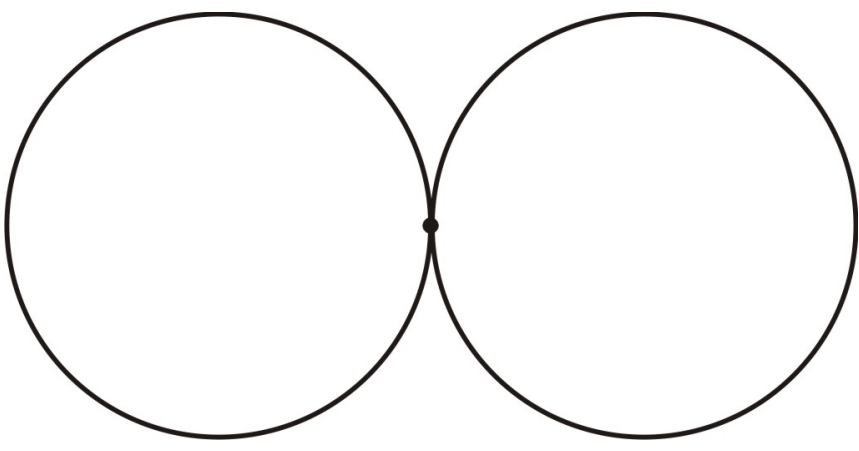

Definição 1.3.15. Dada uma coleção de esferas $S^{n_{1}}, S^{n_{2}}, \ldots, S^{n_{k}}$, o wedge $S^{n_{1}} \vee S^{n_{2}} \vee$ $\cdots \vee S^{n_{k}}$ é chamado de buquê de esferas. 


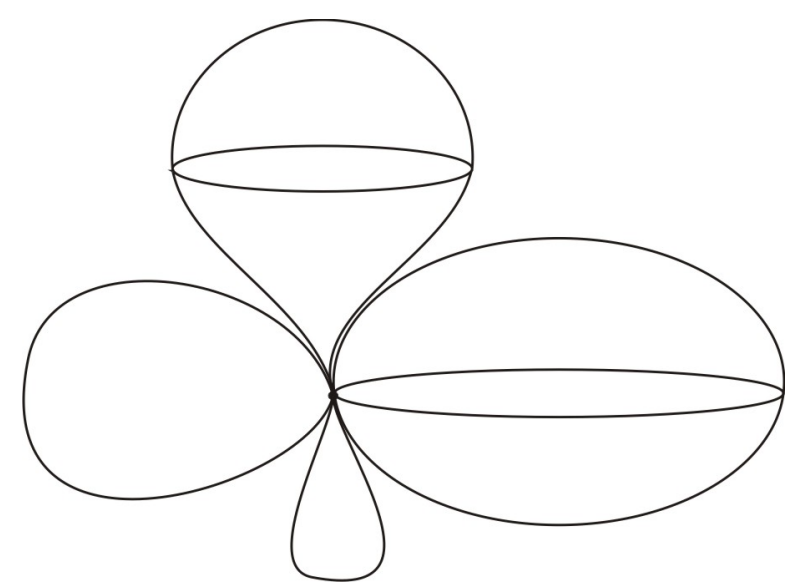

\subsubsection{Homologia Singular}

Dados $x, y \in \mathbb{R}^{n}$, lembramos que o segmento de reta que liga $x$ a $y$ é definido como o conjunto $[x, y]=\{(1-t) x+t y ; 0 \leq t \leq 1\}$. Dizemos que um conjunto $C \subset \mathbb{R}^{n}$ é convexo quando para quaisquer $x, y \in C$ tem-se $[x, y] \subset C$. É um exercício simples verificar que uma interseção arbitrária de conjuntos convexos é ainda um conjunto convexo. O fecho convexo do conjunto $A \subset \mathbb{R}^{n}$ é a interseção de todos os conjuntos convexos de $\mathbb{R}^{n}$ que contêm $A$.

Um $p$-simplexo $s$ em $\mathbb{R}^{n}$ é o fecho convexo de um conjunto de $p+1$ pontos $\left\{x_{0}, \ldots, x_{p}\right\}$ tal que $x_{1}-x_{0}, \ldots, x_{p}-x_{0}$ são linearmente independentes. Note que esta última condição é independente da escolha do ponto $x_{0}$, ou seja, é equivalente a afirmar que os vetores $x_{0}-x_{i}, \ldots, x_{i-1}-x_{i}, x_{i+1}-x_{i}, \ldots, x_{p}-x_{i}$ são linearmente independentes, para cada i. De fato, assumindo que $x_{1}-x_{0}, \ldots, x_{p}-x_{0}$ são linearmente independentes, sejam $\alpha_{0}, \ldots, \alpha_{i-1}, \alpha_{i+1}, \ldots, \alpha_{p}$ tais que

$$
\alpha_{0}\left(x_{0}-x_{i}\right)+\cdots+\alpha_{i-1}\left(x_{i-1}-x_{i}\right)+\alpha_{i+1}\left(x_{i+1}-x_{i}\right)+\cdots+\alpha_{p}\left(x_{p}-x_{i}\right)=0 .
$$


Temos então:

$$
\begin{aligned}
0= & \alpha_{0}\left(x_{0}-x_{i}\right)+\cdots+\alpha_{i-1}\left(x_{i-1}-x_{i}\right)+\alpha_{i+1}\left(x_{i+1}-x_{i}\right)+\cdots+\alpha_{p}\left(x_{p}-x_{i}\right) \\
= & \alpha_{0} x_{0}-\alpha_{0} x_{0}+\alpha_{1} x_{1}-\alpha_{1} x_{0}+\cdots+\alpha_{i-1} x_{i-1}-\alpha_{i-1} x_{0}+\alpha_{i+1} x_{i+1}-\alpha_{i+1} x_{0}+\cdots+ \\
& +\alpha_{p} x_{p}-\alpha_{p} x_{0}+\left(\alpha_{0}+\cdots+\alpha_{i-1}+\alpha_{i+1}+\cdots+\alpha_{p}\right) x_{0}- \\
& \quad-\left(\alpha_{0}+\cdots+\alpha_{i-1}+\alpha_{i+1}+\cdots+\alpha_{p}\right) x_{i} \\
= & \alpha_{1}\left(x_{1}-x_{0}\right)+\cdots+\alpha_{i-1}\left(x_{i-1}-x_{0}\right)+\alpha_{i+1}\left(x_{i+1}-x_{0}\right)+\cdots+\alpha_{p}\left(x_{p}-x_{0}\right)+ \\
& +\left(\alpha_{0}+\cdots+\alpha_{i-1}+\alpha_{i+1}+\cdots+\alpha_{p}\right)\left(x_{0}-x_{i}\right) \\
\Rightarrow & \alpha_{1}=\cdots=\alpha_{i-1}=\alpha_{i+1}=\cdots=\alpha_{p}=0 \text { e } \alpha_{0}+\cdots+\alpha_{i-1}+\alpha_{i+1}+\cdots+\alpha_{p}=0 \\
\Rightarrow & \alpha_{0}=\cdots=\alpha_{i-1}=\alpha_{i+1}=\cdots=\alpha_{p}=0 .
\end{aligned}
$$

Lema 1.3.4. Seja $\left\{x_{0} \ldots, x_{p}\right\} \subset \mathbb{R}^{n}$. As seguintes afirmações são equivalentes:

1. $x_{1}-x_{0}, \ldots, x_{p}-x_{0}$ são linearmente independentes.

2. Se $\sum s_{i} x_{i}=\sum t_{i} x_{i}$ e $\sum s_{i}=\sum t_{i}$, então $s_{i}=t_{i}$ para $i=0, \ldots, p$.

Demonstração. Suponha que a afirmação (1) é verdadeira. Temos:

$$
\begin{aligned}
0 & =\sum s_{i} x_{i}-\sum t_{i} x_{i} \\
& =\sum\left(s_{i}-t_{i}\right) x_{i} \\
& =\sum\left(s_{i}-t_{i}\right) x_{i}-\left(\sum s_{i}-\sum t_{i}\right) x_{0} \\
& =\sum\left(s_{i}-t_{i}\right) x_{i}-\sum\left(s_{i}-t_{i}\right) x_{0} \\
& =\sum\left(s_{i}-t_{i}\right)\left(x_{i}-x_{0}\right)
\end{aligned}
$$

Da independência linear de $x_{1}-x_{0}, \ldots, x_{p}-x_{0}$ segue que $s_{i}-t_{i}=0$ para $i \geq 1$. Então,

$$
s_{0}-t_{0}=\sum\left(s_{i}-t_{i}\right)=\sum s_{i}-\sum t_{i}=0 .
$$

Reciprocamete, suponha agora que a afirmação (2) é verdadeira. Dados $\alpha_{1}, \ldots, \alpha_{p}$ 
$\operatorname{com} \sum_{i=1}^{p} \alpha_{i}\left(x_{i}-x_{0}\right)=0$, temos:

$$
0=\sum \alpha_{i}\left(x_{i}-x_{0}\right)=\sum \alpha_{i} x_{i}-\left(\sum \alpha_{i}\right) x_{0}
$$

Fazendo $s_{0}=0, s_{1}=\alpha_{1}, \ldots, s_{p}=\alpha_{p}$ e $t_{0}=\sum \alpha_{i}, t_{1}=\ldots=t_{p}=0$, temos pela igualdade acima que $\sum s_{i} x_{i}=\sum t_{i} x_{i}$. Além disso, é claro que $\sum s_{i}=\sum t_{i}$. Por (2) segue que $s_{i}=t_{i}$ para $i=0, \ldots, p$, ou seja, $\alpha_{1}=\cdots=\alpha_{p}=0$. Portanto, os vetores $x_{1}-x_{0}, \ldots, x_{p}-x_{0}$ são linearmente independentes.

Dados $x_{0}, \ldots, x_{p} \in \mathbb{R}^{n}$, o fecho convexo do conjunto $\left\{x_{0}, \ldots, x_{p}\right\}$ tem a seguinte caracterização: é o conjunto de todos os pontos da forma $\sum t_{i} x_{i}$, com $0 \leq t_{i} \leq 1 \mathrm{e} \sum t_{i}=1$. Não é difícil verificar que o conjunto $\left\{y=\sum t_{i} x_{i} ; 0 \leq t_{i} \leq 1\right.$ e $\left.\sum t_{i}=1\right\}$ é convexo e contém $\left\{x_{0}, \ldots, x_{p}\right\}$. Observando que todo conjunto convexo $C$ contém todos os pontos da forma $\sum_{i=1}^{k} t_{i} p_{i}$, com $0 \leq t_{i} \leq 1, \sum_{i=1}^{k} t_{i}=1, p_{i} \in C$, para todo inteiro positivo $k$ (isto pode ser feito por indução sobre $k$ ), segue que o fecho convexo de $\left\{x_{0}, \ldots, x_{p}\right\}$ contém o conjunto $\left\{y=\sum t_{i} x_{i} ; 0 \leq t_{i} \leq 1\right.$ e $\left.\sum t_{i}=1\right\}$. Deste modo, pelo Lema 1.3.4 temos o seguinte resultado:

Proposição 1.3.14. Se o p-simplexo singular s é o fecho convexo de $\left\{x_{0}, \ldots, x_{p}\right\}$, então todo ponto de s tem uma única representação da forma $\sum t_{i} x_{i}$, onde $t_{i} \geq 0$ para todo $i$ e $\sum t_{i}=1$.

Os pontos $x_{i}$ são chamados de vértices de $s$. Por esta proposição, podemos identificar os pontos de $s$ com $(p+1)$-uplas $\left(t_{0}, \ldots, t_{p}\right) \in \mathbb{R}^{p+1}$ com uma escolha adequada das coordenadas $t_{i}$.

Se foi dada uma ordem específica aos vértices de $s$, então $s$ é um simplexo ordenado.

Seja então $s$ um simplexo ordenado com vértices $x_{0}, x_{1}, \ldots, x_{p}$. Defina $\sigma_{p}$ como o conjuntos de todos os pontos $\left(t_{0}, t_{1}, \ldots, t_{p}\right) \in \mathbb{R}^{p+1}$ com $\sum t_{i}=1$ e $t_{i} \geq 0$ para cada $i$. Se uma função

$$
f: \sigma_{p} \rightarrow s
$$

é dada por $f\left(t_{o}, \ldots, t_{p}\right)=\sum t_{i} x_{i}$, então $f$ é contínua. Além disso, pela unicidade da representação e pelo fato de $\sigma_{p}$ e $s$ serem espaços de Hausdorff compactos, segue que 
$f$ é um homeomorfismo. Assim, cada $p$-simplexo ordenado é naturalmente homeomorfo a $\sigma_{p}$. Observe que $\sigma_{p}$ é um $p$-simplexo ordenado com vértices $x_{0}^{\prime}=(1,0, \ldots, 0), x_{1}^{\prime}=$ $(0,1, \ldots, 0), \ldots, x_{p}^{\prime}=(0, \ldots, 0,1) . \quad \sigma_{p}$ é chamado de $p$-simplexo padrão com a ordem natural.

Definição 1.3.16. Seja $X$ um espaço topológico. Um p-simplexo singular em $X$ é uma função contínua

$$
\phi: \sigma_{p} \rightarrow X
$$

Se $\phi$ é um $p$-simplexo singular e $i$ é um inteiro com $0 \leq i \leq p$, definimos um $(p-1)$ complexo singular $\partial_{i}(\phi)$ por

$$
\partial_{i}(\phi)\left(t_{0}, \ldots, t_{p-1}\right)=\phi\left(t_{0}, \ldots, t_{i-1}, 0, t_{i}, \ldots, t_{p-1}\right) .
$$

$\partial_{i}(\phi)$ é a $i$-ésima face de $\phi$.

Se $f: X \rightarrow Y$ é uma função contínua e $\phi$ é um $p$-simplexo singular em $X$, definimos um $p$-simplexo singular $f_{\#}(\phi)$ em $Y$ por $f_{\#}(\phi)=f \circ \phi$. Observe que se $g: Y \rightarrow W$ é contínua e $i d_{X}: X \rightarrow X$ é a aplicação identidade,

$$
(g \circ f)_{\#}(\phi)=g_{\#}\left(f_{\#}(\phi)\right) \text { e }\left(i d_{X}\right)_{\#}(\phi)=\phi
$$

Na sequência, vamos lembrar abaixo alguns resultados da teoria de grupos que nos serão úteis.

Definição 1.3.17. Um grupo abeliano $G$ é chamado livre se existe um subconjunto $A \subset G$ tal que todo elemento g em $G$ tem uma única representação

$$
g=\sum_{x \in A} n_{x} \cdot x
$$

onde $n_{x}$ é um inteiro que é não nulo apenas para um número finito de $x$ em $A$. O conjunto $A$ é dito uma base para $G$.

Observação 1.3.3. Dado um conjunto arbitrário $A$, podemos construir um grupo abeliano livre com base $A$ da seguinte maneira: Seja $F(A)$ o conjunto de todas as funções de $A$ em 
$\mathbb{Z}$ tais que $f(x) \neq 0$ apenas para um número finito de elementos de A. Definimos uma operação em $F(A)$ por $(f+g)(x)=f(x)+g(x)$. Então $F(A)$ é um grupo abeliano. Para cada $a \in A$ definimos uma função $f_{a}$ em $F(A)$ por

$$
f_{a}(x)=\left\{\begin{array}{l}
1, \quad \text { se } x=a \\
0, \quad \text { caso contrário . }
\end{array}\right.
$$

Então $\left\{f_{a} ; a \in A\right\}$ é uma base para $F(A)$ como um grupo abeliano livre. Identificando a com $f_{a}$ completamos a construção.

Observe que se $G$ é um grupo abeliano livre e $H$ é um grupo abeliano, então toda função $f: A \rightarrow H$ pode ser estendida unicamente a um homomorfismo $f: G \rightarrow H$ por

$$
f\left(\sum_{x \in A} n_{x} \cdot x\right)=\sum_{x \in A} n_{x} \cdot f(x) .
$$

Agora podemos definir um dos principais objetos de estudo que são as chamadas cadeias singulares.

Definição 1.3.18. Se $X$ é um espaço topológico, defina $S_{n}(X)$ como o grupo abeliano livre cuja base é o conjunto de todos os n-simplexos singulares de $X$. Um elemento de $S_{n}(X)$ é chamado de $n$-cadeia singular de $X$ e tem a forma

$$
\sum_{\phi} n_{\phi} \cdot \phi
$$

onde $n_{\phi}$ é um inteiro, diferente de zero apenas para um número finito de $\phi$.

Uma vez que o $i$-ésimo operador face $\partial_{i}$ é uma função do conjunto de $n$-simplexos singulares no conjunto de $(n-1)$-simplexos singulares, existe uma única extensão a um homomorfismo

$$
\partial_{i}: S_{n}(X) \rightarrow S_{n-1}(X)
$$

dada por $\partial_{i}\left(\sum n_{\phi} \cdot \phi\right)=\sum n_{\phi} \cdot \partial_{i}(\phi)$. Definimos o operador de bordo como o homomorfismo

$$
\partial: S_{n}(X) \rightarrow S_{n-1}(X)
$$


dado por

$$
\partial=\partial_{0}-\partial_{1}+\partial_{2}-\cdots+(-1)^{n} \partial_{n}=\sum_{i=0}^{n}(-1)^{i} \partial_{i} .
$$

Proposição 1.3.15. A composição $\partial \circ \partial \mathrm{em}$

$$
S_{n}(X) \stackrel{\partial}{\longrightarrow} S_{n-1}(X) \stackrel{\partial}{\longrightarrow} S_{n-2}
$$

é nula.

Um elemento $c \in S_{n}(X)$ é chamado de $n$-ciclo se $\partial(c)=0$. Um elemento $d \in S_{n}(X)$ é chamado de $n$-bordo se $d=\partial(e)$ para algum $e \in S_{n+1}(X)$. Uma vez que $\partial$ é um homomorfismo, seu núcleo, o conjunto de todos os $n$-ciclos, é um subgrupo de $S_{n}(X)$ denotado por $Z_{n}(X)$. Similarmente, a imagem $\partial$ em $S_{n}(X)$ é o subgrupo $B_{n}(X)$ de todos os $n$-bordos.

Observe que a Proposição 1.3.15 implica que $B_{n}(X) \subset Z_{n}(X)$ é um subgrupo.

Definição 1.3.19. O grupo quociente

$$
H_{n}(X)=Z_{n}(X) / B_{n}(X)
$$

é o n-ésimo grupo de homologia singular de $X$.

Observação 1.3.4. Também podemos definir os grupos de homologia reduzida de um espaço topológico $X$, denotados por $\tilde{H}_{n}(X)$, e tais grupos se relacionam com os grupos de homologia singular da seguinte forma:

$$
H_{n}(X)= \begin{cases}\tilde{H}_{n}(X), & n>0 \\ \tilde{H}_{0}(X) \oplus \mathbb{Z}, & n=0\end{cases}
$$

Observação 1.3.5. 1. Quando X é uma variedade diferenciável, $X$ é conexa se, e somente se, $H_{0}(X)=\mathbb{Z}$

2. Dado $X$ um $C W$ complexo de dimensão $n$ é possivel provar que $H_{k}(X)=0$ para $k>n$. O leitor interessando pode encontrar uma prova em [18], página 13\%, Lema 1.2.1. 
Considerando $X$ um espaço com o tipo de homotopia de um CW complexo de dimensão $n$, é conhecido que todos os grupos de homologia $H_{k}(X)$, para todo $k$, são finitamente gerados. Segue da classificação de grupos abelianos finitamente gerados ${ }^{1}$ que $H_{k}(X)=$ $\mathbb{Z}^{\beta_{k}} \oplus T$, em que $T$ é o grupo de torção. Os números $\beta_{k}$ é conhecido como posto (ou rank) do grupo $H_{k}(X)$ ou o $k$-ésimo número de Betti do espaço $X$.

Definição 1.3.20. Seja $X$ um espaço topológico que tem o tipo de homotopia de um $C W$ complexo de dimensão n. Definimos a característica de Euler de $X$ por:

$$
\chi(X)=\Sigma_{i=0}^{n}(-1)^{i} \beta_{i}
$$

Observamos que a definição acima é equivalente à Definição 1.3.14 feita anteriormente.

Finalizaremos esta seção lembrando alguns resultados da Topologia Algébrica que utilizaremos no capítulo 2, seção 2 .

Proposição 1.3.16. ([15], página 126) Seja $X$ um espaço topológico Hausdorff e $\mathcal{S}(X)$ a suspensão de $X$. Então

$$
H_{q}(\mathcal{S}(X))= \begin{cases}H_{q-1}(X), & q \geq 0 \\ \tilde{H}_{0}(X), & q=1 \\ \mathbb{Z}, & q=0\end{cases}
$$

Teorema 1.3.2. (Teorema de Hurewicz - [18], página 366) Se um espaço $X$ é $(n-1)$ conexo, com $n \geq 2$, então $H_{i}(X)=0$, para $0<i<n$ e $\pi_{n}(X)=H_{n}(X), H_{0}(X)=\mathbb{Z}$.

\subsection{Noções básicas da Topologia Diferencial}

Definição 1.4.1. Uma fibração suave localmente trivial é uma quádrupla $(B, X, F, p)$, onde $B, X, F$ são variedades suaves, $X$ é conexo e $p: B \rightarrow X$ é uma submersão suave sobrejetiva, satisfazendo as seguintes condições: para cada $x \in X$ existe uma vizinhança $V_{x}$ de $x$ em $X$ e um difeomorfismo $h: V_{x} \times F \rightarrow p^{-1}\left(V_{x}\right)$ tal que $p \circ h(a, b)=a$, para todo

\footnotetext{
${ }^{1}$ Uma referência para este fato é [14], página 309.
} 
$a \in V_{x}, b \in F$. Ou seja, o diagrama abaixo é comutativo:

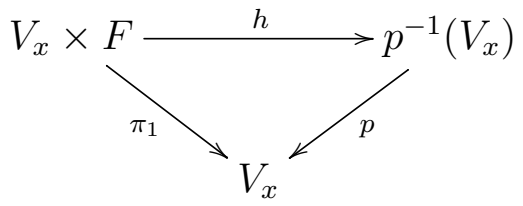

Aqui, $\pi_{1}: V_{x} \times F \rightarrow V_{x}$ é a projeção na primeira coordenada $\pi_{1}(a, b)=a$.

$A$ variedade $B$ é chamada de espaço fibrado ou espaço total da fibração. A variedade $X$ é o espaço base, ou simplesmente base, da fibração. A variedade $F$ é a fibra da fibração. Além disso, a aplicação p é chamada de aplicaçãa projeção da fibração.

Quando pudermos escolher $V_{x}=X$, diremos que o fibrado é trivial. Daí, $B$ é difeomorfo a $X \times F$.

Segue da definição acima que para cada $x \in X$, a variedade $p^{-1}(x)$ é difeomorfa a $F$, chamada de fibra sobre o ponto $x$ de $X$.

Observação 1.4.1. Quando não houver confusão de notação, nos referiremos à projeção $p: B \rightarrow X$ simplesmente por fibração suave.

Exemplo 1.4.1. Dadas $X$ e $Y$ variedades suaves, e considerando $\pi_{1}: X \times Y \rightarrow Y$ a projeção como acima, temos claramente que $\pi_{1}$ é uma fibração suave trivial.

Exemplo 1.4.2. Um exemplo de fibrado suave localmente trivial é o fibrado tangente de uma variedade suave $M$, denotado por TM. A variedade TM é definida como a união disjunta dos espaços tangentes à variedade $M$ :

$$
T M=\bigcup_{p \in M}\left(\{p\} \times T_{p} M\right) \text {, onde } T_{p} M \text { é o espaço tangente a } M \text { no ponto } p .
$$

A aplicação projeção $\pi: T M \rightarrow M, \pi(p, v)=p$ é uma submersão suave e sobrejetora, o que torna a quádrupla $\left(T M, M, \pi, T_{p} M\right)$ um fibrado suave localmente trivial. Por este motivo a variedade TM recebe o nome de fibrado tangente da variedade $M$.

Sendo $M=\mathbb{R}^{n}$, então $T \mathbb{R}^{n}=\mathbb{R}^{n} \times \mathbb{R}^{n}$. Além disso, para qualquer aberto $U \subset R^{n}$ temos $T U=U \times \mathbb{R}^{n}$. 
Definição 1.4.2. Dada uma variedade suave $M$, dizemos que $M$ é paralelizável quando a quádrupla $\left(T M, M, \pi, T_{p} M\right)$ é um fibrado suave trivial.

Um problema interessante é encontrar as dimensões $n$ para as quais as esferas $S^{n}$ são paralelizáveis. Esse problema foi resolvido por R. Bott e J. Milnor em [7] onde provaram que as únicas esferas paralelizáveis são $S^{1}, S^{3}$ e $S^{7}$.

Dada $f: M \rightarrow N$ uma aplicação suave entre duas variedades, podemos definir a aplicação suave $\widehat{f}: T M \rightarrow T N$, dada por $\widehat{f}(p, v):=(f(p), d f(p)(v))$, onde $d f(p): T_{p} M \rightarrow$ $T_{f(p)} N$ é a aplicação diferencial de $f$ em $p$.

Decorre da definição que, dadas $f: M \rightarrow N$ e $g: N \rightarrow P$, temos $\widehat{g \circ f}=\widehat{g} \circ \widehat{f}$. Além disso, se considerarmos a aplicação identidade $i d_{M}: M \rightarrow M$, então $\widehat{i d_{M}}=i d_{T M}$. Logo, temos que se $f: M \rightarrow N$ é um difeomorfismo, então $\widehat{f}: T M \rightarrow T N$ também é um difeomorfismo. Portanto, se $M$ e $N$ são variedades difeomorfas, $M$ é paralelizável se, e somente se, $N$ é paralelizável.

Observe que, para todo $n$ natural, $n \geq 1$, temos que a aplicação $f: S^{n} \times \mathbb{R} \rightarrow$ $\mathbb{R}^{n+1} \backslash\{0\}$, dada por $f(x, t)=e^{t} . x$ é um difeomorfismo cuja inversa é dada por $f^{-1}$ : $\mathbb{R}^{n+1} \backslash\{0\} \rightarrow S^{n} \times \mathbb{R}, f^{-1}(y)=\left(\frac{y}{\|y\|}, \ln (\|y\|)\right)$. Com isso, podemos enunciar o seguinte resultado.

Proposição 1.4.1. Para todo $n$, a variedade $S^{n} \times \mathbb{R}$ é paralelizável.

Demonstração. De fato, sendo $\mathbb{R}^{n+1} \backslash\{0\}$ aberto em $\mathbb{R}^{n}$ temos que o fibrado tangente $T\left(\mathbb{R}^{n+1} \backslash\{0\}\right)=\left(\mathbb{R}^{n+1} \backslash\{0\}\right) \times \mathbb{R}^{n+1}$. Portanto, o resultado decorre do difeomorfismo acima.

Definição 1.4.3. Dado um fibrado suave localmente trivial $(B, X, F, p)$, uma seção transversal do fibrado é uma aplicação suave $f: X \rightarrow B$ tal que $p \circ f(x)=x$ para todo $x \in X$. Uma seção transversal suave da quádrupla $\left(T M, M, \pi, T_{p} M\right)$ é chamado um campo de vetores suave tangente à variedade $M$.

Decorre da definição que uma seção transversal de um fibrado é uma aplicação injetiva.

Definição 1.4.4. Sejam $M, N$ variedades suaves e $f: M \rightarrow N$ uma aplicação contínua. Dizemos que $f$ é uma aplicação própria se para todo $K \subset N$ compacto, tem-se $f^{-1}(K) \subset$ 
$M$ é compacto.

Se $f: M \rightarrow N$ é contínua e $M$ é compacta, então claramente $f$ é própria. De forma geral, podemos caracterizar as aplicações próprias da seguinte maneira.

Proposição 1.4.2. Dadas variedade suaves $M, N$ e uma aplicação contínua $f: M \rightarrow N$, as seguintes afirmações são equivalentes:

1. f é própria.

2. $f$ é uma aplicação fechada e, para cada $y \in N$, o conjunto $f^{-1}(y)$ é compacto.

3. Se $\left(x_{k}\right)$ é uma sequência em $M$ tal que a sequência correspondente $\left(f\left(x_{k}\right)\right)$ em $N$ é convergente, então $\left(x_{k}\right)$ tem uma subsequência convergente.

O resultado a seguir é uma condição suficiente para uma aplicação entre duas variedades ser a projeção de um fibrado suave localmente trivial. Vamos enunciá-lo para variedades com bordo, que será a situação mais geral que nos deparamos neste trabalho. Utilizaremos a seguinte notação: dada $M$ variedade com bordo, denotaremos o bordo de $M$ por $\partial M$.

Definição 1.4.5. Sejam $M$ e $N$ variedades suaves, com $\partial M$ não vazio. Uma aplicação $f: M \rightarrow N$ é uma submersão suave se as restrições $f: M \backslash \partial M \rightarrow N$ e $f: \partial M \rightarrow N$ forem ambas submersões.

Teorema 1.4.1. (Teorema de Ehresmann) Sejam $M, N$ variedades suaves com $N$ conexa, $M$ com bordo e $f: M \rightarrow N$ uma submersão suave, sobrejetora e própria. Então a quádrupla $\left(M, N, f^{-1}(y), f\right)$ é um fibrado suave localmente trivial.

Para finalizar, enunciaremos um resultado que relaciona a teoria de homotopia e fibração, que vamos aplicar no capítulo 4, seção 4.3. Para isso, introduzimos mais uma definição.

Definição 1.4.6. Sejam $M_{i}, i=1,2,3$, módulos sobre um anel comutativo com unidade $R, f: M_{1} \rightarrow M_{2}$ e $g: M_{2} \rightarrow M_{3} R$-homomorfismo. Diremos que uma sequência

$$
0 \rightarrow M_{1} \stackrel{f}{\rightarrow} M_{2} \stackrel{g}{\rightarrow} M_{3} \rightarrow 0
$$


é exata (curta) se $f$ for injetora, g for sobrjetora e $\operatorname{Im}(f)=\operatorname{Ker}(g)$. Mais geralmente, dada uma família de $R$-módulos $\left\{M_{n}\right\}_{n \in \mathbb{Z}}$ e uma familia de $R$-homomorfismos $\left\{f_{n}\right.$ : $\left.M_{n} \rightarrow M_{n-1}\right\}_{n \in \mathbb{Z}}$, diremos que a sequência

$$
\cdots \rightarrow M_{n+1} \stackrel{f_{n+1}}{\rightarrow} M_{n} \stackrel{f_{n}}{\rightarrow} M_{n-1} \rightarrow \cdots
$$

é exata (longa) se $\operatorname{Im}\left(f_{n+1}\right)=\operatorname{Ker}\left(f_{n}\right)$, para todo $n$.

Proposição 1.4.3. Dada uma sequência exata $0 \rightarrow M_{1} \stackrel{f}{\rightarrow} M_{2} \stackrel{g}{\rightarrow} M_{3} \rightarrow 0$, as seguintes afirmações são equivalentes:

1. Existe um $R$-homomorfismo $\phi: M_{2} \rightarrow M_{1}$ tal que $\phi \circ f=I d_{M_{1}}$;

2. Existe um R-homomorfismo $\psi: M_{3} \rightarrow M_{2}$ tal que $g \circ \psi=I d_{M_{3}}$.

Além disso, se uma das condiçôes acima for verdadeira temos o seguinte isomorfismo

$$
M_{2}=M_{1} \oplus M_{3}
$$

Teorema 1.4.2. Seja $(M, B, F, p)$ uma fibração suave com $M, B$ e $F$ variedades conexas. Então a seguinte sequência (longa) é exata

$$
\cdots \rightarrow \pi_{n}(F) \rightarrow \pi_{n}(M) \rightarrow \pi_{n}(B) \rightarrow \pi_{n-1}(F) \cdots \rightarrow \pi_{0}(B) \rightarrow \pi_{0}(F) \rightarrow 0 .
$$




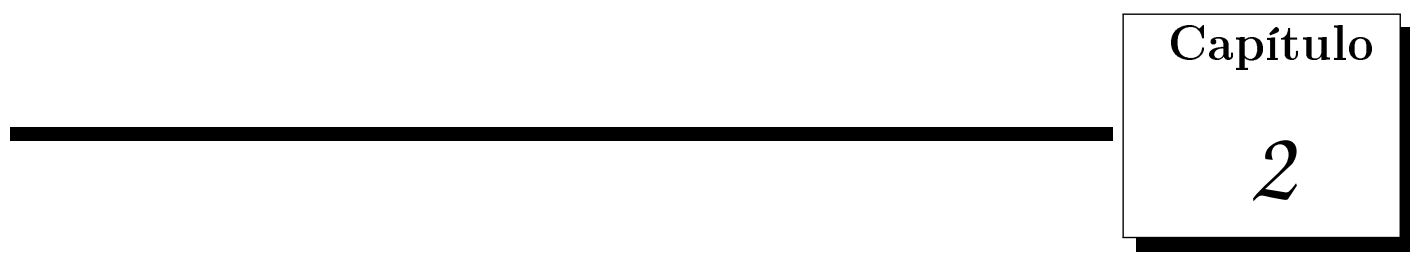

\section{Singularidades de germes de funções}

\section{holomorfas}

Neste capítulo apresentamos os principais resultados relacionados ao estudo da topologia local de singularidades de funções holomorfas. A ferramenta usada neste estudo é a fibração de Milnor. Mais recentemente, em [37] e [35] foi trabalhado com detalhes a existência desta fibração. Aqui, discutimos o Teorema da Fibração de Milnor e as informações topológicas da fibra desta fibração e do link, tanto no caso de singularidade isolada como não-isolada, acompanhando nosso estudo com exemplos a fim de facilitar a compreensão dos resultados.

\subsection{O teorema da fibração de Milnor}

Em [27] (1968), John Milnor estudou a topologia da singularidade de uma função holomorfa $^{1} f: U \subseteq \mathbb{C}^{n+1} \rightarrow \mathbb{C}, U \subseteq \mathbb{C}^{n+1}$ aberto, $0 \in U$ com $f(0)=0$, através de uma

\footnotetext{
${ }^{1} \mathrm{Na}$ verdade, Milnor trabalhou com germes de funções polinomiais, mas seu trabalho pode ser naturalmente estendido para funções holomorfas, a partir da versão analítica do Lema de Seleção da Curva.
} 
fibração localmente trivial dada por

$$
\begin{aligned}
\phi: S_{\varepsilon} \backslash K & \rightarrow S^{1} \\
z & \mapsto \frac{f(z)}{|f(z)|}
\end{aligned}
$$

para $\varepsilon>0$ suficientemente pequeno, onde $S_{\varepsilon}=\left\{z \in \mathbb{C}^{n+1} ;\|z\|=\varepsilon\right\}, S^{1}$ é a esfera unitária de $\mathbb{C}$ e $K=S_{\varepsilon} \cap f^{-1}(0)$ conhecido como o link da singularidade. Denotamos também $V=f^{-1}(0) \cap U$. Nessa notação, o principal teorema de Milnor pode ser enunciado da seguinte forma:

Teorema 2.1.1. Teorema da Fibração de Milnor. Seja $f:\left(\mathbb{C}^{n+1}, 0\right) \rightarrow(\mathbb{C}, 0)$ um germe de função holomorfa. Então, para $\varepsilon>0$ suficientemente pequeno, $S_{\varepsilon} \backslash K$ é um fibrado suave localmente trivial sobre $S^{1}$, com aplicação projeção $\phi: S_{\varepsilon} \backslash K \rightarrow S^{1}$ dada por

$$
\phi(z)=\frac{f(z)}{|f(z)|}
$$

No caso $\Sigma(f) \subset\{0\}$, podemos encontrar o $\varepsilon>0$ citado no Teorema acima aplicando o Lema 3.1.1. No caso geral, o leitor interessado pode encontrar na referência [27], Lema 4.1 e 4.2 .

Vários passos são percorridos no caminho da demonstração deste teorema. O primeiro deles consiste em caracterizar os pontos críticos da aplicação $\phi$ para garantir a existência de $\varepsilon>0$ para o qual $\phi$ não tem pontos críticos, ou seja, que torna $\phi$ uma submersão. Em seguida, escrevendo $\phi(z)=e^{i \theta(z)}$ definimos localmente uma função suave $\theta$ e, usando um campo vetorial tangente em $S_{\varepsilon} \backslash K$ e suas curvas integrais, podemos mostrar que $\theta$ ao longo de uma curva integral $p(t)$ é da forma

$$
\theta(p(t))=t+\text { constante, } \forall t \in \mathbb{R}
$$

o que implica a sobrejetividade da aplicação $\phi$. Segue então que para todo $\theta$ as fibras $F_{\theta}=\phi^{-1}\left(e^{i \theta}\right)$ são subvariedades $2 n$-dimensionais de $S_{\varepsilon}$. Finalmente, usamos o fluxo do campo vetorial para gerar um difeomorfismo que torna $\phi$ uma aplicação projeção da fibração. A ideia é que o campo foi escolhido adequadamente de modo que suas curvas 
integrais sejam transversais às fibras $F_{\theta}=\phi^{-1}\left(e^{i \theta}\right)$.

Dado um $\varepsilon_{0}>0$ tal que para todo $0<\varepsilon \leq \varepsilon_{0}$ temos que $\phi$ é uma projeção da fibração acima, dizemos que $\varepsilon_{0}$ é um raio de Milnor de $\phi$ e que as variedades $F_{\theta}$ são as fibras de Milnor da aplicação $\phi$.

No decorrer deste capítulo manteremos sempre a mesma notação: $f$ é um representante do germe de função holomorfa $f:\left(\mathbb{C}^{n+1}, 0\right) \rightarrow(\mathbb{C}, 0)$ e $V=f^{-1}(0) \cap U$, para algum aberto $U$. Os espaços $K, S_{\varepsilon}$ e a aplicação $\phi$ são como no Teorema da Fibração.

\subsection{Topologia da fibra e do link}

Suponha agora que $0 \in \Sigma(f)$, o conjunto crítico da função $f$ podendo ser isolado ou não. Ainda em [27], Milnor usou técnicas da teoria de Morse e provou os resultados gerais a seguir sobre a topologia da fibra.

Teorema 2.2.1. A fibra $F_{\theta}$ é paralelizável e tem o tipo de homotopia de um $C W$ complexo de dimensão (no máximo) n.

Teorema 2.2.2. O link $K$ é $(n-2)$-conexo.

A ideia usada por Milnor para provar o Teorema 2.2.1. foi aplicar a teoria de Morse para estudar a função $|f|$ restrita a $F_{\theta}$. Por motivo de conveniência, estuda-se a função

$$
\begin{aligned}
a_{\theta}: F_{\theta} & \rightarrow \mathbb{R} \\
z & \mapsto \log |f(z)| .
\end{aligned}
$$

Claramente, $|f|$ restrita a $F_{\theta}$ tem os mesmos pontos críticos de $a_{\theta}$. É feita uma caracterização destes pontos críticos e, usando uma expressão para a segunda derivada de $a_{\theta}$, conclui-se que o índice de Morse de $a_{\theta}$ em um ponto crítico é $\geq n$. Usa-se a caracterização dos pontos críticos de $a_{\theta}$ para verificar que tais pontos críticos estão num compacto de $F_{\theta}$. Assim, podemos encontrar uma função de Morse $s_{\theta}: F_{\theta} \rightarrow \mathbb{R}_{+}$com índice $\geq n$ que coincide com $|f|$ quando $|f(z)|$ é suficientemente pequeno e, além disso, tem número finito de pontos críticos. A partir desta função, definimos uma função própria e limitada 
inferiormente

$$
\begin{aligned}
g_{\theta}: F_{\theta} & \rightarrow \mathbb{R} \\
z & \mapsto-\log s_{\theta}(z),
\end{aligned}
$$

que tem índice de Morse $\geq n$ e, aplicando resultados da Teoria de Morse, ${ }^{2}$ concluímos que $F_{\theta}$ tem o mesmo tipo de homotopia de um CW-complexo finito de dimensão $\leq n$.

Usando um raciocínio análogo e trabalhando com a aplicação

$$
\begin{aligned}
a: S_{\varepsilon} \backslash K & \rightarrow \mathbb{R} \\
z & \mapsto \log |f(z)|
\end{aligned}
$$

podemos provar que o espaço total $S_{\varepsilon} \backslash K$ tem o tipo de homotopia de um CW-complexo finito de dimensão $\leq n+1$.

Na prova do Teorema 2.2.2 começamos com uma vizinhança conveniente de $K$

$$
N(K)=\left\{z \in S_{\varepsilon} ;|f(z)| \leq \eta\right\}
$$

que é uma variedade suave com bordo para todo $\eta>0$ suficientemente pequeno, pois $0 \in \mathbb{C}$ é um valor crítico isolado. Usando a aplicação $s: S_{\varepsilon} \backslash K \rightarrow \mathbb{R}$ obtida de $a: S_{\varepsilon} \backslash K \rightarrow \mathbb{R}$ pelo mesmo procedimento feito para obter a $s_{\theta}$ tratada acima, podemos verificar usando ferramentas da topologia algébrica que a esfera $S_{\varepsilon}$ tem o mesmo tipo de homotopia de um complexo contruído a partir da vizinhança $N(K)$ adjuntando células de dimensão $\geq n$. Daí, conclui-se que

$$
\pi_{i}(N(K))=\pi_{i}\left(S_{\varepsilon}\right)=0, \text { para } i \leq n-2 .
$$

Observando que $K$ é um retrato de uma vizinhança absoluta e sendo um conjunto algébrico, segue por Łojasiewicz que ele é triangularizável - [22]. Logo obtemos que $K$ é um retrato da vizinhança $N(K)$, para $\eta$ suficientemente pequeno, e segue que $\pi_{i}(K)$ também é trivial para $i \leq n-2$.

Posteriormente, Kato e Matsumoto mostraram em [19] (1973) o resultado abaixo que indica que a topologia da fibra está intimamente relacionada com a degeneração da singularidade.

\footnotetext{
${ }^{2}$ Teorema 3.5 de [26], página 20.
} 
Teorema de Kato-Matsumoto: Suponha que $0 \in \mathbb{C}^{n+1}$ seja um ponto crítico do germe de função holomorfa $f:\left(\mathbb{C}^{n+1}, 0\right) \rightarrow(\mathbb{C}, 0)$. Seja $s=\operatorname{dim}_{\mathbb{C}, 0} \Sigma(f)$ a dimensão complexa do germe do conjunto crítico $\Sigma(f)$ em 0 . Então, a fibra de Milnor $F_{\theta}$ é $(n-s-1)$ conexa.

Observe que não podemos concluir pelo teorema que $\pi_{n-s}(F) \neq 0$ como mostra o Exemplo 2.2.1 a seguir.

Exemplo 2.2.1. Seja $f: \mathbb{C}^{3} \rightarrow \mathbb{C}$ dada por $f\left(z_{1}, z_{2}, z_{3}\right)=z_{1} z_{2}^{3}+z_{3}^{2}$. Temos:

$$
\begin{gathered}
\nabla f\left(z_{1}, z_{2}, z_{3}\right)=\left(z_{2}^{3}, 3 z_{1} z_{2}^{2}, 2 z_{3}\right)=0 \Leftrightarrow z_{2}=z_{3}=0 \\
\Rightarrow \Sigma(f)=\left\{\left(z_{1}, 0,0\right) ; z_{1} \in \mathbb{C}\right\}
\end{gathered}
$$

e $\operatorname{dim}_{\mathbb{C}, 0} \Sigma(f)=1$. Pelo Teorema de Sakamoto ([38]), $F_{\theta}$ é homotopicamente equivalente à suspensão da fibra da função $h\left(z_{1}, z_{2}\right)=z_{1} z_{2}^{3}$. Como $h$ é dada por um polinômio quasihomogêneo, é suficiente estudar a fibra $h^{-1}(1)$ :

$$
h\left(z_{1}, z_{2}\right)=z_{1} z_{2}^{3}=1 \Leftrightarrow z_{1}=\frac{1}{z_{2}^{3}},
$$

de modo que a fibra $h^{-1}(1)$ é difeomorfa a $\mathbb{C}^{*}$ pela aplicação

$$
\begin{aligned}
\mathbb{C}^{*} & \rightarrow h^{-1}(1) \\
t & \mapsto\left(\frac{1}{t^{3}}, t\right) .
\end{aligned}
$$

Dai $\pi_{1}\left(h^{-1}(1)\right)=\pi_{1}\left(\mathbb{C}^{*}\right)=\pi_{1}\left(S^{1}\right)$. Logo,

$$
\pi_{1}(F)=\pi_{1}\left(\mathcal{S}\left(h^{-1}(1)\right)\right)=\pi_{1}\left(\mathcal{S}\left(S^{1}\right)\right)=\pi_{1}\left(S^{2}\right)=0 .
$$

Portanto, $\pi_{n-s}(F)=0$.

O seguinte exemplo mostra que o Teorema de Kato-Matsumoto não pode ser melhorado, ou seja, ele fornece o nível máximo de conexidade que podemos obter. 
Exemplo 2.2.2. Considere $f: \mathbb{C}^{3} \rightarrow \mathbb{C}$ definida por $f\left(z_{1}, z_{2}, z_{3}\right)=z_{1} z_{2}$. Temos:

$$
\begin{gathered}
\nabla f\left(z_{1}, z_{2}, z_{3}\right)=\left(z_{2}, z_{1}, 0\right)=0 \Leftrightarrow z_{1}=z_{2}=0 \\
\Rightarrow \Sigma(f)=\left\{\left(0,0, z_{3}\right) ; z_{3} \in \mathbb{C}\right\},
\end{gathered}
$$

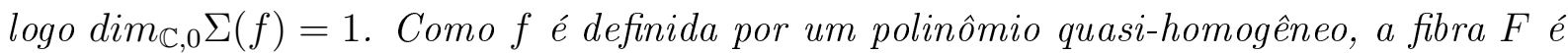
difeomorfa $a f^{-1}(1)$ :

$$
f\left(z_{1}, z_{2}, z_{3}\right)=z_{1} z_{2}=1 \Leftrightarrow z_{2}=\frac{1}{z_{1}}
$$

então podemos ver $f^{-1}(1)$ como o gráfico da função $(t, w) \mapsto \frac{1}{t}$ definida em $\mathbb{C}^{*} \times \mathbb{C}$, que por sua vez é difeomorfo ao domínio. Portanto,

$$
\pi_{1}(F)=\pi_{1}\left(f^{-1}(1)\right)=\pi_{1}\left(\mathbb{C}^{*} \times \mathbb{C}\right)=\pi_{1}\left(S^{1}\right) \neq 0 .
$$

Note que pelo Teorema de Kato-Matsumoto não podemos extrair muitas informações da topologia da fibra quando o conjunto singular tem dimensão grande, ou seja, quanto maior a dimensão de $\Sigma(f)$, menos informações nos é dada por este teorema sobre a conexidade da fibra.

O caso particular em que $s=0$, isto é, quando a singularidade é isolada, foi mostrado por Milnor em [27], página 57, usando técnicas da teoria de Morse. Neste caso, a fibra é $(n-1)$-conexa. Outra informação interessante da topologia da fibra é que seu fecho $\bar{F}_{\theta}$ em $S_{\varepsilon}$ é uma variedade com bordo $2 n$-dimensional cujo interior é $F_{\theta}$ e cujo bordo é o link $K$. Além disso, a variedade $\bar{F}_{\theta}$ está mergulhada em $S_{\varepsilon}$ de modo a ter o mesmo tipo de homotopia de seu complementar $S_{\varepsilon} \backslash \bar{F}_{\theta}$. De fato, sendo $\phi: S_{\varepsilon} \backslash K \rightarrow S^{1}$ uma fibração localmente trivial, segue que a restrição $\phi: S_{\varepsilon} \backslash\left(K \cup F_{\theta}\right) \rightarrow S^{1} \backslash\left\{e^{i \theta}\right\}$ é ainda uma fibração localmente trivial, e como $S^{1} \backslash\left\{e^{i \theta}\right\}$ é contrátil, esta última fibração é trivial. Portanto, $S_{\varepsilon} \backslash \bar{F}_{\theta}=S_{\varepsilon} \backslash\left(K \cup F_{\theta}\right)$ é difeomorfo a $F_{\theta} \times\left(S^{1} \backslash\left\{e^{i \theta}\right\}\right)$, que tem o tipo de homotopia de $F_{\theta}$, por $S^{1} \backslash\left\{e^{i \theta}\right\}$ ser contrátil, e temos

$$
S_{\varepsilon} \backslash \bar{F}_{\theta}=S_{\varepsilon} \backslash\left(K \cup F_{\theta}\right) \equiv F_{\theta} \times\left(S^{1} \backslash\left\{e^{i \theta}\right\}\right) \equiv F_{\theta} \equiv \bar{F}_{\theta} .
$$


A última equivalência segue do fato conhecido em topologia algébrica que uma variedade com bordo tem o mesmo tipo de homotopia que sua parte aberta ${ }^{3}$.

Milnor provou também que a fibra tem o tipo de homotopia de um buquê de esferas n-dimensionais, e o número de esferas no buquê é conhecido como o número de Milnor da singularidade. Observe que, sendo

$$
F_{\theta} \equiv \underbrace{S^{n} \vee S^{n} \vee \cdots \vee S^{n}}_{\mu}
$$

onde $\mu$ representa o número de esferas, podemos utilizar a decomposição celular da seguinte forma: sendo as esferas unidas num único ponto comum (digamos, $p$ ), particionamos o buquê de esferas de modo que uma componente desta partição consiste de tal ponto comum e as demais componentes consistem cada uma de uma esfera menos o ponto p. Assim, nesta decomposição temos $\mu$-esferas menos um ponto (ou seja, $\mu$ células de dimensão $n$ ) e uma célula de dimensão 0. Deste modo, chegamos facilmente à seguinte fórmula para a característica de Euler-Poincaré da fibra:

$$
\chi\left(F_{\theta}\right)=1+(-1)^{n} \mu .
$$

De forma geral, quando a dimensão do conjunto singular é positivo, não podemos esperar que a fibra de Milnor tenha o tipo de homotopia de um buquê de esferas, como podemos verificar no exemplo a seguir.

Exemplo 2.2.3. Considere $f:\left(\mathbb{C}^{3}, 0\right) \rightarrow(\mathbb{C}, 0), f\left(z_{1}, z_{2}, z_{3}\right)=z_{1} z_{2} z_{3}$. Com cálculos simples, vemos que o conjunto singular de $f$ é

$$
\Sigma(f)=\left\{\left(z_{1}, 0,0\right) ; z_{1} \in \mathbb{C}\right\} \cup\left\{\left(0, z_{2}, 0\right) ; z_{2} \in \mathbb{C}\right\} \cup\left\{\left(0,0, z_{3}\right) ; z_{3} \in \mathbb{C}\right\} .
$$

Vamos analisar a fibra $F=f^{-1}(1)$ :

$$
z_{1} z_{2} z_{3}=1 \Leftrightarrow z_{3}=\frac{1}{z_{1} z_{2}}
$$

\footnotetext{
${ }^{3}$ Uma prova desta afirmação pode ser encontrada em [43], página 297.
} 
e vemos que $F$ é difeomorfa a $\mathbb{C}^{*} \times \mathbb{C}^{*}$ pela aplicação

$$
\begin{aligned}
\mathbb{C}^{*} \times \mathbb{C}^{*} & \rightarrow F \\
\left(z_{1}, z_{2}\right) & \mapsto\left(z_{1}, z_{2}, \frac{1}{z_{1} z_{2}}\right)
\end{aligned}
$$

Dai, F tem o tipo de homotopia do toro $S^{1} \times S^{1}$.

Devido à complexidade que a fibra pode assumir quando a singularidade não é isolada, as pesquisas têm sido feitas acrescentando-se mais hipóteses, ou seja, considerando-se casos especiais. Por exemplo, quando o conjunto singular tem dimensão um $(s=1)$, o Teorema de Kato-Matsumoto nos diz que a fibra é $(n-2)$-conexa, e pelo Teorema de Hurewicz (Teorema 1.3.2) temos que $H_{j}(F)=0$, para todo $0<j \leq n-2$. Logo, restam somente os grupos $H_{n-1}(F)$ e $H_{n}(F)$, sendo que este último já sabemos que é livre de torção (ver [18], p. 132, exercício 22).

De forma geral, podemos escrever $H_{n-1}(F)=\mathbb{Z}^{b_{n-1}} \oplus T$, onde $T$ é o subgrupo de torção. Em [29] foi feita a seguinte construção:

Para cada par de inteiros $(l, d), \operatorname{com} l \geq 1$ e $d \geq 2$, fixe uma aplicação $f_{l, d}: S^{l} \rightarrow S^{l}$ de grau $d$. Considere a bola fechada unitária $(l+1)$-dimensional $B^{l+1}$ e a inclusão $i: S^{l} \hookrightarrow$ $B^{l+1}$. Definimos o espaço $T(l, d)$ por

$$
T(l, d)=B^{l+1} \sqcup S^{l} / \sim,
$$

onde $\sim$ é a relação de equivalência que identifica cada ponto $x \in S^{l}=\partial B^{l+1} \operatorname{com} f_{l, d}(x) \in$ $S^{l}$. Nestas condições foi provado que o tipo de homotopia do espaço $T(l, d)$ não depende da escolha da aplicação $f_{l, d}$. Além disso, se $l \geq 2$, o espaço $T(l, d)$ tem as seguintes propriedades:

(i) $\tilde{H}_{q}(T(l, d), \mathbb{Z})=0$ para $q \neq l$,

(ii) $\tilde{H}_{q}(T(l, d), \mathbb{Z})=\mathbb{Z}_{d}$ para $q=l$.

Com isto foi provado o seguinte resultado topológico:

Teorema 2.2.3. Considere um $C W$-complexo $n$-dimensional $X$ que é $(n-2)$-conexo $(n \geq 3)$. Seja $T$ o grupo de torção de $H_{n-1}(X, \mathbb{Z})$ e escreva $T$ como soma direta de 
grupos cíclicos, $T=\oplus_{i} G_{i}$. Seja $b_{i}$ o i-ésimo número de Betti de $X$. Então X tem o tipo de homotopia do buquê

$$
\left(\bigvee_{b_{n}} S^{n}\right) \vee\left(\bigvee_{b_{n-1}} S^{n-1}\right) \vee\left(\bigvee_{i} T\left(n-1,\left|G_{i}\right|\right)\right)
$$

onde $\left|G_{i}\right|$ é a ordem do grupo $G_{i}$.

Como caso particular, segue o seguinte resultado.

Corolário 2.2.1. Nas condições do Teorema 2.2.3 a fibra tem o tipo de homotopia de um buquê de esferas se, e somente se, o grupo de homologia $H_{n-1}(F)$ é livre de torção.

Naturalmente, nem sempre podemos esperar que o grupo $H_{n-1}(F)$ seja livre de torção. De fato, em [], os autores exibem uma família de singularidades de funções holomorfas onde isso não acontece.

Observe que a hipótese na dimensão $n \geq 3$ no Teorema 2.2.3 não pode ser removida, como mostra o Exemplo 2.2.3 feito anteriormente.

Existem várias generalizações do teorema do buquê para singularidades não isoladas. O leitor interessado pode consultar por exemplo [45] e [41] e suas referências.

A seguir apresentaremos alguns exemplos que vão nos ajudar numa melhor compreensão dos resultados acima.

Exemplo 2.2.4. Seja $f: \mathbb{C}^{4} \rightarrow \mathbb{C}, f\left(z_{1}, z_{2}, z_{3}, z_{4}\right)=z_{1} z_{2} z_{3}+z_{4}^{2}$. Aplicando o Teorema de Sakamoto, temos que a fibra de Milnor F é homotopicamente equivalente à suspensão da fibra de Milnor do Exemplo 2.2.3; ou seja, a fibra de Milnor é homotopicamente equivalente a $\mathcal{S}\left(S^{1} \times S^{1}\right)$. Agora aplicando a Proposição 1.3.16, obtemos que $H_{0}(F)=H_{3}(F)=\mathbb{Z}$, $H_{1}(F)=0$ e $H_{2}(F)=\mathbb{Z} \oplus \mathbb{Z}$. Portanto, $r k\left(H_{2}(F)\right)=2, r k\left(H_{3}(F)\right)=1$ e, desde que os subgrupos de torção são nulos, podemos concluir do Teorema 2.2.3 que a fibra tem o tipo de homotopia de um buquê de esferas dado por $S^{2} \vee S^{2} \vee S^{3}$.

Exemplo 2.2.5. (Guarda-chuva de Whitney) Seja $f: \mathbb{C}^{4} \rightarrow \mathbb{C}, f\left(z_{1}, z_{2}, z_{3}, z_{4}\right)=z_{1}^{2}-$ $z_{3} z_{2}^{2}+z_{4}^{2}$. Novamente por Sakamoto, podemos dividir o estudo da topologia da fibra em duas partes: 
(1) a fibra da função $g\left(z_{1}, z_{2}, z_{3}\right)=z_{1}^{2}-z_{3} z_{2}^{2}$ é homotopicamente equivalente à suspensão da fibra de Milnor de $h\left(z_{2}, z_{3}\right)=-z_{3} z_{2}^{2}$;

(2) por fim, a fibra de Milnor de f é homotopicamente equivalente à suspensão da fibra de Milnor da função g do passo (1).

Pela parte (1), sendo h dada por um polinômio quasi-homogêneo, com a mesma ideia dos exemplos anteriores obtemos que a fibra de Milnor é difeomorfa a $\mathbb{C}^{*}$, logo homotopicamente equivalente a $S^{1}$. Daí concluímos que a fibra de Milnor de g é homotopicamente equivalente a $\mathcal{S}\left(S^{1}\right)=S^{2}$. E finalmente por (2) temos que a fibra de Milnor $F$ é homotopicamente equivalente a $\mathcal{S}\left(S^{2}\right)=S^{3}$.

Observe que neste caso segue diretamente que $H_{2}(F)=0$ (logo livre de torção) $e$ $r k\left(H_{3}(F)\right)=1$. 


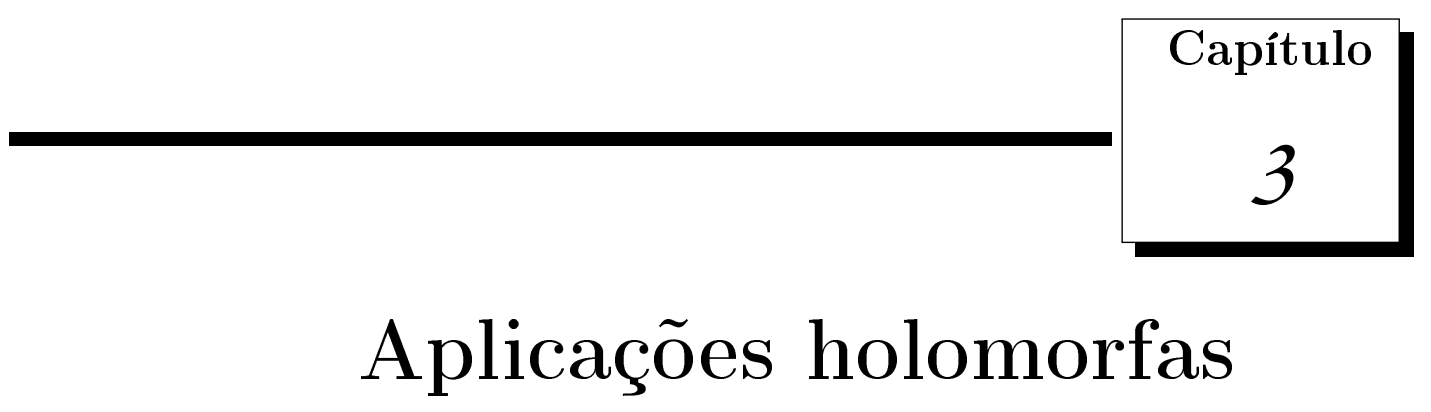

Neste capítulo apresentaremos a existência da fibração de Milnor para germes de aplicações holomorfas $f:\left(\mathbb{C}^{n+k}, 0\right) \rightarrow\left(\mathbb{C}^{k}, 0\right)$ em uma situação especial, a saber quando $f$ define uma ICIS. Nosso estudo será desenvolvido da seguinte forma: na primeira seção são apresentados resultados gerais para germes de conjuntos analíticos. Na segunda seção restringiremos ao nosso caso de interesse formulando assim o teorema da fibração. Na terceira seção exibimos alguns resultados sobre a topologia da fibra e do link, seguidos de exemplos na última seção.

A principal referência para este capítulo é $[23]^{1}$. Novamente, para facilitar a leitura do texto, utilizamos a mesma notação para um germe e seu representante.

\subsection{O link de uma Singularidade Isolada}

Seja $X$ um conjunto analítico em um aberto $U \subset \mathbb{C}^{N}$. Suponha que $x \in X$ é tal que $X \backslash\{x\}$ é regular de dimensão constante $n$ (ou seja, $X \backslash\{x\}$ é uma variedade suave de dimensão $n$ ). No decorrer desta seção, vamos manter esta notação. O objetivo desta seção é mostrar que numa vizinhança de $x$, o conjunto $X$ é homeomorfo a um cone sobre uma variedade suave, que é chamada de link da singularidade e é único, a menos de difeomorfismo.

\footnotetext{
${ }^{1}$ Nesta referência, o autor desenvolve o estudo com mais generalidade do que fazemos aqui.
} 
Considere uma aplicação $r: X \rightarrow[0, \infty)$ que é a restrição de uma função real-analítica $\tilde{r}$ definida em uma vizinhança de $X$ em $U$ e tal que $r^{-1}(0)=\{x\}$. Nestas condições, dizemos que a função $r$ define o ponto $x \mathrm{em} X$. Para cada $\varepsilon>0$, fixamos a seguinte notação:

$$
X_{r \leq \varepsilon}=\{x \in X ; r(x) \leq \varepsilon\} .
$$

De modo análogo, $X_{r<\varepsilon}, X_{r=\varepsilon}, X_{0<r<\varepsilon}$, etc.

Um primeiro resultado interessante que obtemos por $x$ ser um ponto singular isolado (ou regular) do conjunto $X$ é o seguinte.

Lema 3.1.1. Seja $r: X \rightarrow[0, \infty)$ uma função que define o ponto $x$ em $X$. Então 0 não é um ponto de acumulação de valores críticos de $\left.r\right|_{X \backslash\{x\}}$.

Demonstração. Como o problema é de caráter local, podemos supor, diminuindo $U$ se necessário, que $X=\left\{y \in U: f_{1}(y)=\ldots=f_{k}(y)=0\right\}$, onde $f_{i}, i=1, \cdots, k$, são funções holomorfas definidas em $U$. Como $X \backslash\{x\}$ é regular de dimensão $n$, segue que para todo $y \in$ $X \backslash\{x\}$, o sistema $\left\{d f_{1}(y), \ldots, d f_{k}(y)\right\}$ tem posto $N-n$. Considere o conjunto $Y \subset X$ que consiste dos pontos $y$ de $X$ nos quais o sistema $\left\{d \tilde{r}(y), d f_{1}(y), \ldots, d f_{k}(y), d \bar{f}_{1}(y), \ldots, d \bar{f}_{k}(y)\right\}$ tem posto $\leq 2(N-n) .{ }^{2}$ Podemos ver que $Y$ é um subconjunto real-analítico de $X$ e $x \in Y$. Além disso, $Y \backslash\{x\}$ é o conjunto crítico de $\left.r\right|_{X \backslash\{x\}}$. É suficiente então mostrar que $x$ é ponto isolado de $Y$.

Suponha por absurdo que $x$ é ponto de acumulação de $Y$. Então, pelo Lema de Seleção da Curva, existe uma curva real-analítica $\gamma:[0, \delta) \rightarrow \mathbb{C}^{N}$ tal que $\gamma(0)=x$ e $\gamma(t) \in Y \backslash\{x\}$ se $t \neq 0$. Observe que o sistema $\left\{d f_{1}(y), \ldots, d f_{k}(y), d \bar{f}_{1}(y), \ldots, d \bar{f}_{k}(y)\right\}$ tem posto $2(N-$ $n)$, então $y \in Y$ significa que $d \tilde{r}(y)$ é combinação linear de $d f_{1}(y), \ldots, d f_{k}(y), d \bar{f}_{1}(y), \ldots$, $d \bar{f}_{k}(y)$, e então está no espaço normal a $X \backslash\{x\}$ em $y$. Temos então que

$$
\frac{d(r \circ \gamma)}{d t}(t)=\left\langle d r(\gamma(t)), \frac{d \gamma}{d t}(t)\right\rangle=0
$$

donde segue que $r \circ \gamma$ é constante, e $(r \circ \gamma)(t)=(r \circ \gamma)(0)=r(x)=0$ para todo $t$. Uma vez que $r^{-1}(0)=\{x\}$, isto implica que $\gamma(t)=x$ para todo $t$, o que contradiz a hipótese

\footnotetext{
${ }^{2}$ Se tratam das derivadas reais. Ver [21].
} 
de $\gamma(t) \in Y \backslash\{x\}$ para $t \neq 0$.

Os seguintes lemas serão bastante úteis em resultados posteriores.

Lema 3.1.2. Seja $r: X \rightarrow[0, \infty)$ uma função que define o ponto $x$ em $X$. Então existe $\varepsilon>0$ tal que $X_{r \leq \varepsilon}$ é compacto e $\left.r\right|_{X \backslash\{x\}}$ não tem valor crítico em $(0, \varepsilon]$. Consequentemente, $X_{r=\varepsilon}$ é uma subvariedade real-analítica compacta de $X$.

Demonstração. Pelo Lema 3.1.1, existe $\varepsilon^{\prime}>0$ tal que $\left(0, \varepsilon^{\prime}\right)$ não tem valor crítico de $\left.r\right|_{X \backslash\{x\}}$. Considere o aberto $U^{\prime}=U \cap \tilde{r}^{-1}\left(-\varepsilon^{\prime}, \varepsilon^{\prime}\right)$. Então $X^{\prime}=X \cap U^{\prime}$ é um conjunto analítico em $U^{\prime}$ que contém $x$, e é equivalente (como germe) a $X$. Podemos então trabalhar com este representante $X^{\prime}$ do germe. Afim de facilitar notação, continuaremos usando a notação $X$ para o representante do germe.

Como $\left.r\right|_{X \backslash\{x\}}$ não tem ponto crítico, então é crescente, e portanto injetiva. Seja $B$ uma bola fechada com centro $x$ que está contida em $U$. Então $r(B)=[0, \varepsilon] \subset\left[0, \varepsilon^{\prime}\right)$ não tem valor crítico de $\left.r\right|_{X \backslash\{x\}}$ e $X_{r \leq \varepsilon}=B$ é compacto.

O conjunto $X_{r=\varepsilon}$ é imagem inversa de valor regular, portanto subvariedade suave de $X \backslash\{x\}$. Sendo um fechado do compacto $X_{r \leq \varepsilon}$, é também compacto.

Lema 3.1.3. Sejam $r, r^{\prime}: X \rightarrow[0, \infty)$ aplicações que definem $x$ em $X$. Então existe uma vizinhança $V$ de $x$ em $X$ tal que $d r(y)$ e $d r^{\prime}(y)$ não apontam em direções opostas, para nenhum $y \in V \backslash\{x\}$. Ou seja, se $d r(y)$ e $d r^{\prime}(y)$ são linearmente independentes sobre $\mathbb{R}$, então o produto interno euclidiano $\Re\left\langle d r(y), d r^{\prime}(y)\right\rangle$ é não-negativo.

Demonstração. Assim como no Lema 3.1.1, vamos supor que $X$ é definido pelas funções holomorfas $f_{1}, \ldots, f_{k}$. Suponha também que $r$ e $r^{\prime}$ são restrições das funções reaisanalíticas $\tilde{r}$ e $\tilde{r}^{\prime}$, respectivamente. Seja $Y$ o subconjunto de $X$ formado pelos pontos $y$ tais que $d r(y)$ e $d r^{\prime}(y)$ são linearmente independentes sobre $\mathbb{R}$. Ou seja, $Y$ é o conjunto dos $y \in X$ nos quais o sistema $\left\{d f_{1}(y), \ldots, d f_{k}(y), d \bar{f}_{1}(y), \ldots, d \bar{f}_{k}(y), d \tilde{r}(y), d \tilde{r}^{\prime}(y)\right\}$ tem posto $<2(N-n+1)$. Vemos então que $Y$ é um subconjunto real-analítico de $X$.

Suponha por absurdo que existem pontos $y$ em $Y \backslash\{x\}$ arbitrariamente próximos de $x$ nos quais $\Re\left\langle d r(y), d r^{\prime}(y)\right\rangle<0$. Segue do Lema de Seleção da Curva que existe uma curva 
real-analítica $\gamma:[0, \delta) \rightarrow Y$ tal que $\gamma(0)=x$ e $\Re\left\langle d r(\gamma(t)), d r^{\prime}(\gamma(t))\right\rangle<0$ para $t>0$. Daí $d r^{\prime}(\gamma(t))=\lambda(t) d r(\gamma(t))$ para algum $\lambda(t)<0$ sempre que $t>0$. Mas

$$
\frac{d\left(r^{\prime} \circ \gamma\right)}{d t}(t)=\left\langle d r^{\prime}(\gamma(t)), \frac{d \gamma}{d t}(t)\right\rangle=\lambda(t)\left\langle d r(\gamma(t)), \frac{d \gamma}{d t}(t)\right\rangle=\lambda(t) \frac{d(r \circ \gamma)}{d t}(t)
$$

donde segue que $r$ e $r^{\prime}$ não podem crescer simultaneamente ao longo de $\gamma$, ou seja, ou $r$ ou $r^{\prime}$ deve permanecer constante ao longo de uma curva suave que passa por $x$. Isto é uma contradição, pois $r^{-1}(0)=r^{-1}(0)=\{x\}$.

A seguir apresentaremos três resultados do Capítulo 2 de [23]. Omitiremos suas provas por serem bastante técnicas e não darem contribuição significativa no desenvolvimento do trabalho.

Lema 3.1.4. Sejam $r, r^{\prime}: X \rightarrow[0, \infty)$ aplicações que definem $x$ em $X$. Seja $\varepsilon>0$ tal que $X_{r \leq \varepsilon}$ é compacto e contido numa vizinhança $V$ como no Lema 3.1 .3 e tal que $r$ e $r^{\prime}$ não têm ponto crítico em $X_{r \leq \varepsilon}$. Então existe um campo vetorial suave $v$ definido em uma vizinhança de $X_{r \leq \varepsilon}$ que satisfaz $\langle d r(y), v(y)\rangle=-1 e\left\langle d r^{\prime}(y), v(y)\right\rangle<0$ e é tangente a $X$.

Proposição 3.1.1. Nas condições do Lema 3.1.2, existe um homeomorfismo $H$ do cone de $X_{r=\varepsilon}$ sobre $X_{r \leq \varepsilon}$ tal que $\frac{1}{\varepsilon} r \circ H$ é a projeção sobre $[0,1]$.

Definição 3.1.1. A subvariedade $X_{r=\varepsilon}$ de $X$ é chamada de link de $x$ em $X$.

Proposição 3.1.2. Sejam $r, r^{\prime}: X \rightarrow[0, \infty)$ aplicações que definem $x$ em $X$. Existe $\varepsilon>0$ tal que

(i) $X_{r \leq \varepsilon}$ é compacto e não contém pontos críticos de $\left.r\right|_{X \backslash\{x\}}$.

(ii) $S e \varepsilon^{\prime}>0$ é tal que

$$
X_{r^{\prime} \leq \varepsilon^{\prime}} \subset X_{r<\varepsilon}
$$

então $X_{r^{\prime} \leq \varepsilon^{\prime}}$ é compacto, não contém pontos críticos de $\left.r^{\prime}\right|_{X \backslash\{x\}}$ e existe um difeomorfismo de $X_{r \leq \varepsilon, r^{\prime} \geq \varepsilon^{\prime}}$ sobre $[0,1] \times X_{r=\varepsilon}$ que leva $X_{r=\varepsilon}$ sobre $\{0\} \times X_{r=\varepsilon}$ e leva $X_{r^{\prime}=\varepsilon^{\prime}}$ sobre $\{1\} \times$ $X_{r=\varepsilon}$.

Corolário 3.1.1. Quaisquer duas estruturas de link em X são difeomorfas. 
Demonstração. Segue diretamente da proposição anterior: as devidas restrições do difeomorfismo dado pela Proposição 3.1.2 promovem difeomorfismos de $X_{r=\varepsilon}$ em $\{0\} \times X_{r=\varepsilon}$ e de $X_{r=\varepsilon}$ em $\{1\} \times X_{r=\varepsilon}$.

Segue do Corolário acima que, a menos de difeomorfismo, o link independe da função que define $x$ em $X$ que tomamos. Podemos então tomar a função $r$ como a restrição a $X$ da função suave

$$
\begin{aligned}
\tilde{r}: \mathbb{C}^{N} & \longrightarrow[0, \infty) \\
y & \longmapsto r(y)=\|y-x\|^{2}
\end{aligned}
$$

sem perda de generalidade.

\subsection{Teorema da fibração}

No capítulo 2 estudamos a existência da fibração de Milnor para funções holomorfas. Convém então perguntar em que situação podemos ter uma fibração similar para aplicações holomorfas. Geralmente essa fibração não existe, como mostra o seguinte exemplo:

Exemplo 3.2.1. Seja $f:\left(\mathbb{C}^{3}, 0\right) \rightarrow\left(\mathbb{C}^{2}, 0\right)$ dada por $f(x, y, z)=(x, x y z)$. Com cálculos simples vemos que

$$
\begin{gathered}
\Sigma(f)=\left\{(x, y, z) \in \mathbb{C}^{3} ; x=0\right\} \cup\left\{(x, y, z) \in \mathbb{C}^{3} ; z=0\right\}, \\
f(\Sigma(f))=\{(x, 0) ; x \in \mathbb{C}\} .
\end{gathered}
$$

Para cada $\delta \neq 0$, tem-se $f^{-1}((\delta, 0))=\{(\delta, y, 0) ; y \in \mathbb{C}\} \cup\{(\delta, 0, z) ; z \in \mathbb{C}\}$. Por outro lado, para cada $\delta_{1} \neq 0, \delta_{2} \neq 0$, tem-se $f^{-1}\left(\left(\delta_{1}, \delta_{2}\right)\right)=\left\{\left(\delta_{1}, y, \frac{\delta_{2}}{\delta_{1} y}\right) ; y \in \mathbb{C}^{*}\right\}$, que é homeomorfo a $\mathbb{C}^{*}$. Sendo assim, não pode haver fibração de Milnor, pois os tipos topológicos das fibras são diferentes.

Uma condição suficiente para a existência de tal fibração é que ela defina uma ICIS. O objetivo desta seção é enunciar e provar o teorema de existência da fibração de Milnor para esta classe de aplicações holomorfas. 
Vamos começar definindo os conceitos necessários para nosso estudo e fixando a notação que vamos usar.

Considere $\mathcal{I}=\left\{f \in \mathcal{O}_{a, N} ; f\right.$ se anula em uma vizinhança de $a$ em $\left.X\right\}$. Tal conjunto é um ideal de $\mathcal{O}_{a, N}$, chamado de ideal do germe $(X, a)$.

Como sabemos que $\mathcal{O}_{a, N}$ é um anel noetheriano, existem funções analíticas $f_{1}, \ldots, f_{k}$ definidas em uma vizinhança de $a$ que geram o ideal do germe $(X, a)$. Por [40], sabemos que a dimensão do germe $(X, a)$ é $\geq N-k$, ou seja, $k \geq N-\operatorname{dim}_{\mathbb{C}}(X, a)$.

Definição 3.2.1. Dizemos que o germe $(X, a)$ é uma interseção completa se o seu ideal possui um sistema de exatamente $N-\operatorname{dim}_{\mathbb{C}}(X, a)$ geradores.

Definição 3.2.2. Seja $f:\left(\mathbb{C}^{n}, 0\right) \rightarrow\left(\mathbb{C}^{k}, 0\right)$ um germe de aplicação holomorfa. Dizemos que $f$ define uma ICIS ${ }^{3}$ na origem se o germe $(V(f), 0)$ é uma interseção completa $e$

$$
V(f) \cap \Sigma(f) \subset\{0\}
$$

onde $V(f)=f^{-1}(0)$.

Dada uma aplicação holomorfa $f=\left(f_{1}, \ldots, f_{k}\right): \mathbb{C}^{n} \rightarrow \mathbb{C}^{k}$, com $f(0)=0$, o conjunto $V(f)=f^{-1}(0)$ é um conjunto analítico em $\mathbb{C}^{n}$, e é claro que a função

$$
\begin{aligned}
r: \mathbb{C}^{n} & \rightarrow \mathbb{R} \\
x & \mapsto\|x\|^{2}
\end{aligned}
$$

define o ponto 0 em $V(f)$. No caso em que $f$ define uma ICIS na origem, todo ponto de $V(f) \backslash\{0\}$ é regular, de modo que $V(f) \backslash\{0\}$ é uma variedade suave. Podemos então aplicar os conceitos e resultados desenvolvidos na seção 1 para o conjunto analítico $V(f)$ e a função $r$ que define o ponto 0 em $V(f)$.

Para cada $\eta>0$, denotamos por $B_{\eta}^{2 k}$ a bola fechada em $\mathbb{C}^{k}$ com centro na origem e raio $\eta$. Denotamos por $D_{f}$ a imagem do conjunto singular de $f$ em $B_{\eta}^{2 k}$, ou seja, $D_{f}=f(\Sigma(f)) \cap B_{\eta}^{2 k}$.

\footnotetext{
${ }^{3}$ Este nome deriva do inglês Isolated Complete Intersection Singularity (interseção completa com singularidade isolada). O conceito de ICIS é mais geral, e pode ser encontrado com a devida generalização em [23].
} 
Teorema 3.2.1. Seja $f$ um germe de ICIS na origem. Então, existe um $\varepsilon_{0}>0$ tal que, para todo $0<\varepsilon \leq \varepsilon_{0}$, existe $\eta=\eta(\varepsilon)$, com $0<\eta \ll \varepsilon \ll 1$ tal que

$$
f: B_{\varepsilon}^{2 n} \cap f^{-1}\left(B_{\eta}^{2 k} \backslash D_{f}\right) \rightarrow B_{\eta}^{2 k} \backslash D_{f}
$$

é a projeção de uma fibração suave localmente trivial.

Demonstração. Pelo Lema 3.1.1, existe $\varepsilon_{0}>0$ tal que a aplicação $\left.r\right|_{V(f)}$ não tem valor crítico em $\left(0, \varepsilon_{0}^{2}\right]$. Ou seja,

$$
\operatorname{rank}\left(\begin{array}{c}
\nabla r(x) \\
\nabla f_{1}(x) \\
\vdots \\
\nabla f_{k}(x)
\end{array}\right)
$$

é maximo para todo $x \in r^{-1}\left(\left(0, \varepsilon_{0}^{2}\right]\right)$. Isto significa que $\left(f^{-1}(0) \backslash\{0\}\right) \pitchfork S_{\varepsilon}^{2 n-1}$ para cada $0<\varepsilon \leq \varepsilon_{0}$, portanto 0 é valor regular da aplicação

$$
f: S_{\varepsilon}^{2 n-1} \rightarrow \mathbb{R}^{2 k}
$$

Então, existe $\eta=\eta(\varepsilon)>0$ tal que, para todo $y \in B_{\eta}^{2 k}, y$ é valor regular de $f: S_{\varepsilon}^{2 n-1} \rightarrow$ $\mathbb{R}^{2 k}$. Assim,

$$
f: S_{\varepsilon}^{2 n-1} \cap f^{-1}\left(B_{\eta}^{2 k}\right) \rightarrow B_{\eta}^{2 k}
$$

é submersão sobrejetora própria. Pelo Teorema de Ehresmann, é fibração suave localmente trivial. Segue então que

$$
f: S_{\varepsilon}^{2 n-1} \cap f^{-1}\left(B_{\eta}^{2 k} \backslash D_{f}\right) \rightarrow B_{\eta}^{2 k} \backslash D_{f}
$$

também é fibração localmente trivial.

Agora, observe que

$$
f: \stackrel{\circ}{B}_{\varepsilon}^{2 n} \cap f^{-1}\left(B_{\eta}^{2 k} \backslash D_{f}\right) \rightarrow \stackrel{\circ}{B}_{\eta}^{2 k} \backslash D_{f}
$$


é submersão. Segue então de (3.2.1) e (3.2.2) serem submersões que

$$
f: B_{\varepsilon}^{2 n} \cap f^{-1}\left(B_{\eta}^{2 k} \backslash D_{f}\right) \rightarrow B_{\eta}^{2 k} \backslash D_{f}
$$

é submersão. Observe que a aplicação

$$
f: B_{\varepsilon}^{2 n} \cap f^{-1}\left(B_{\eta}^{2 k}\right) \rightarrow B_{\eta}^{2 k}
$$

é suave, sobrejetora e própria (por ter domínio compacto), logo a aplicação (3.2.3) também é. De fato, se $K \subset B_{\eta}^{2 k} \backslash D_{f}$ é compacto, então é subconjunto compacto de $B_{\eta}^{2 k}$. Logo, $f^{-1}(K) \cap B_{\varepsilon}^{2 n} \cap f^{-1}\left(B_{\eta}^{2 k}\right)$ é compacto. Mas $K \subset B_{\eta}^{2 k} \backslash D_{f}$, portanto $f^{-1}(K) \subset f^{-1}\left(B_{\eta}^{2 k} \backslash D_{f}\right)$ e então

$$
f^{-1}(K) \cap B_{\varepsilon}^{2 n} \cap f^{-1}\left(B_{\eta}^{2 k}\right)=f^{-1}(K) \cap B_{\varepsilon}^{2 n} \cap f^{-1}\left(B_{\eta}^{2 k} \backslash D_{f}\right) .
$$

Isto prova que a aplicação (3.2.3) é própria. Finalmente, pelo Teorema de Ehresmann, segue que a aplicação (3.2.3) é fibração suave localmente trivial.

Da prova do Teorema 3.2.1 segue o seguinte corolário:

Corolário 3.2.1. Dado um germe de aplicação holomorfa $f:\left(\mathbb{C}^{n}, 0\right) \rightarrow\left(\mathbb{C}^{k}, 0\right)$ que define uma ICIS na origem, existe $\varepsilon_{0}>0$ tal que, para todo $\varepsilon>0$ com $0<\varepsilon \leq \varepsilon_{0}$, existe um $\eta=\eta(\varepsilon)$, com $0<\eta \ll \varepsilon \ll 1$ tal que

$$
f: S_{\varepsilon}^{2 n-1} \cap f^{-1}\left(B_{\eta}^{2 k}\right) \rightarrow B_{\eta}^{2 k}
$$

é fibração trivial.

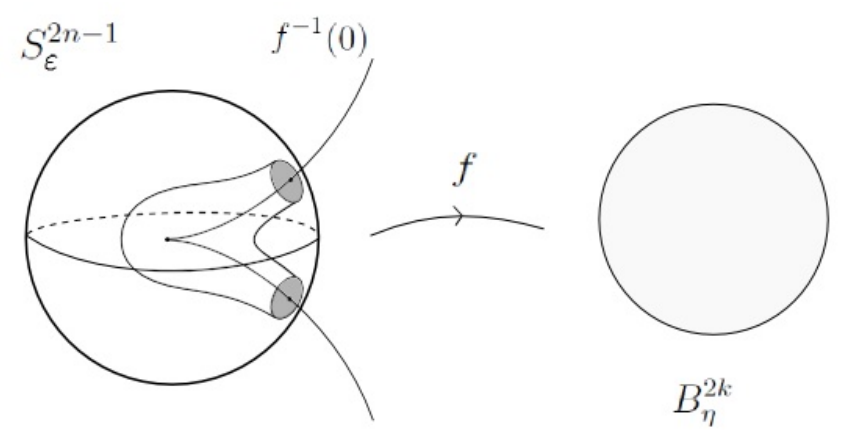


Demonstração. Vimos na prova do Teorema 3.2.1 que a aplicação $f: S_{\varepsilon}^{2 n-1} \cap f^{-1}\left(B_{\eta}^{2 k} \rightarrow\right.$ $B_{\eta}^{2 k}$ é fibração suave localmente trivial. Como a base da fibração é contrátil, segue que a fibração é trivial.

Observação 3.2.1. Nas condições do Corolário 3.2.1, obtemos que o link de $f$ é difeomorfo à fronteira da fibra de Milnor.

Ainda neste caso foi provado por E. Looijenga em [23] que todas as fibras são difeomorfas, têm o tipo de homotopia de um $C W$-complexo de dimensão $\leq n$ e são $(n-1)$-conexas. Logo, seguindo o argumento análogo ao de Milnor foi provado que a fibra tem o tipo de homotopia de um buquê finito de n-esferas. Portanto, ainda neste caso podemos definir o número de Milnor como segue.

Definição 3.2.3. Seja $f:\left(\mathbb{C}^{n}, 0\right) \rightarrow\left(\mathbb{C}^{k}, 0\right), n>k \geq 2$ um germe de ICIS. O número de Milnor de $f$ é definido como o número de esferas que aparecem no buquê da fibra de Milnor da fibração. 


\section{Capítulo}

\section{Singularidades de aplicações reais}

\subsection{As condições (a) e (b) de Milnor}

Neste capítulo usamos as notações e principais resultados de [24]. Mais resultados sobre estudos de singularidades analíticas reais podem ser encontrados em [25, 9, 1].

Sejam $U \subset \mathbb{R}^{n}$ aberto e $f=\left(f_{1}, \ldots, f_{k}\right): U \rightarrow \mathbb{R}^{k}$ uma aplicação suave, com $f(0)=0$. Suponha que $f$ não é constante em uma vizinhança da origem. Denotemos por $X=$ $f^{-1}(0)=V(f) \cap U$. Tomamos novamente a função $r: U \subseteq \mathbb{R}^{n} \rightarrow \mathbb{R}$ dada por $r(x)=\|x\|^{2}$. Considere os conjuntos

$\mathfrak{A}=\left\{x \in U ; \nabla f_{1}(x), \nabla f_{2}(x), \ldots, \nabla f_{k}(x)\right.$ são linearmente independentes $\}$,

$\mathfrak{B}=\left\{x \in U ; \nabla r(x), \nabla f_{1}(x), \nabla f_{2}(x), \ldots, \nabla f_{k}(x)\right.$ são linearmente independentes $\}$

Então $\mathfrak{A}=\Sigma(f)$ e é claro que $\mathfrak{A}$ e $\mathfrak{B}$ são subconjuntos fechados de $U$ com $\mathfrak{A} \subset \mathfrak{B}$.

Definição 4.1.1. i) Dizemos que a aplicação $f$ satisfaz a condição (a) de Milnor em 0 (ou $0 \in \mathbb{R}^{k}$ é um valor crítico isolado de $f$ ) se $0 \notin \overline{\mathfrak{A} \backslash X}$, isto é, se $\Sigma(f) \subset V(f)$ em uma vizinhança de 0 em $U$. Neste caso, se $\varepsilon>0$ é tal que $B_{\varepsilon} \cap(\overline{\mathfrak{A} \backslash X})=\varnothing$, então dizemos que $\varepsilon$ é um (a) raio de Milnor para $f$ em 0 .

ii) Dizemos que a aplicação f satisfaz a condição (b) de Milnor em 0 se 0 é um ponto 
isolado de (ou não está em) $X \cap \overline{\mathfrak{B} \backslash X}$. Neste caso, se $\varepsilon>0$ é tal que $B_{\varepsilon} \cap X \cap(\overline{\mathfrak{B} \backslash X}) \subset$ $\{0\}$, dizemos que $\varepsilon$ é um (b) raio de Milnor para $f$ em 0 .

iii) Se f satisfaz as condições (a) e (b) de Milnor e $\varepsilon>0$ é simultaneamente um (a) raio de Milnor e um (b) raio de Milnor para $f$ em 0, dizemos simplesmente que $\varepsilon$ é um raio de Milnor para $f$ em 0 .

Geometricamente, a condição (b) de Milnor diz que para cada esfera suficientemente pequena, as fibras próximas do link são transversais à esfera.

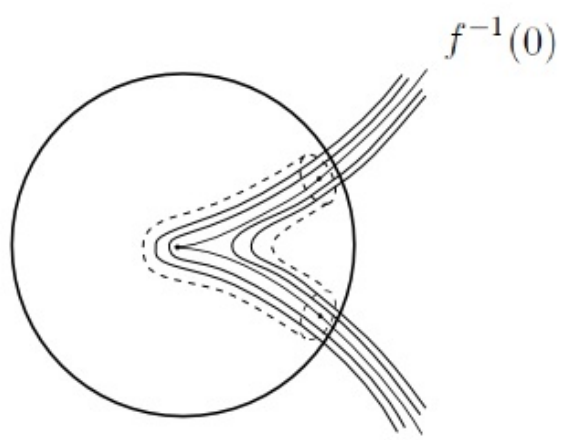

Proposição 4.1.1. Seja $f: U \subseteq \mathbb{R}^{n} \rightarrow \mathbb{R}^{k}$, uma aplicação polinomial, $n \geq k \geq 2,0 \in U$ com $f(0)=0$. Assuma que 0 é um ponto crítico isolado da aplicação $f$. Então, $f$ admite um raio de Milnor na origem.

Demonstração. A condição de Milnor (a) é trivial!

Considere a função $r(x)=\|x\|^{2}$ em $U$. Do Lema 3.1.1 decorre que a variedade $B_{\varepsilon} \cap$ $(V(f) \backslash\{0\})$ é transversal às curvas de nível de $r$ para todo $\varepsilon$ suficientemente pequeno.

Proposição 4.1.2. Suponha que a aplicação $f$ satisfaz a condição (b) de Milnor em 0 e seja $\varepsilon_{0}>0$ um (b) raio de Milnor para $f$ em 0 . Então, para todo $\varepsilon>0$ tal que $0<\varepsilon \leq \varepsilon_{0}$, existe $\delta_{\varepsilon}>0$ tal que a aplicação

$$
H:\left(\stackrel{\circ}{B}_{\varepsilon_{0}} \backslash B_{\varepsilon}\right) \cap f^{-1}\left(\stackrel{\circ}{B}_{\delta_{\varepsilon}} \backslash\{0\}\right) \rightarrow\left(\stackrel{\circ}{B}_{\delta_{\varepsilon}} \backslash\{0\}\right) \times\left(\varepsilon^{2}, \varepsilon_{0}^{2}\right)
$$

dada por $H(x)=\left(f(x),\|x\|^{2}\right)$ é uma submersão própria. 
Demonstração. Para mostrar que $H$ é própria, tome $K \subset\left(\stackrel{\circ}{B}_{\delta_{\varepsilon}} \backslash\{0\}\right) \times\left(\varepsilon^{2}, \varepsilon_{0}^{2}\right)$ compacto. Sendo $\pi: \stackrel{\circ}{B}_{\delta_{\varepsilon}} \times\left(\varepsilon^{2}, \varepsilon_{0}^{2}\right) \rightarrow\left(\varepsilon^{2}, \varepsilon_{0}^{2}\right)$ a projeção na segunda coordenada, temos que $\pi(K)$ é compacto (digamos $\pi(K)=[a, b] \subset\left(\varepsilon^{2}, \varepsilon_{0}^{2}\right)$ ), e então $H^{-1}(K)$ é subconjunto fechado do conjunto $\left\{x \in \stackrel{\circ}{B}_{\varepsilon_{0}} ;\|x\|^{2} \in \pi(K)\right\} \subset B_{\sqrt{b}}$. Portanto, $H^{-1}(K)$ é compacto.

Agora, note que os pontos críticos de $H$ são os pontos de $\mathfrak{B} \cap\left(\stackrel{\circ}{B}_{\varepsilon_{0}} \backslash B_{\varepsilon}\right) \cap f^{-1}\left(\stackrel{\circ}{B}_{\delta_{\varepsilon}} \backslash\{0\}\right)$. Suponha por absurdo que não existe $\delta_{\varepsilon}>0$ que torna $H$ uma submersão. Em outras palavras: existe uma sequência $x_{i}$ em $(\mathfrak{B} \backslash X) \cap\left(\stackrel{\circ}{B}_{\varepsilon_{0}} \backslash B_{\varepsilon}\right)$ tal que $f\left(x_{i}\right) \rightarrow 0$. Como cada $x_{i}$ pertence ao conjunto compacto $B_{\varepsilon_{0}} \backslash \stackrel{\circ}{B}_{\varepsilon}$ então, a menos de subsequência, temos $x_{i} \rightarrow x \in$ $B_{\varepsilon_{0}} \backslash \stackrel{\circ}{B}_{\varepsilon}$. Uma vez que $f\left(x_{i}\right) \rightarrow 0$, temos que $x \in X$. Portanto, $x \in B_{\varepsilon_{0}} \backslash \stackrel{\circ}{B}_{\varepsilon} \cap X \cap(\overline{\mathfrak{B} \backslash X})$, o que é uma contradição, pois estamos supondo que $\varepsilon_{0}$ é um (b) raio de Milnor para $f$ em 0 .

Corolário 4.1.1. Se f satisfaz a condição (b) de Milnor em 0 e $\varepsilon_{0}>0$ é um (b) raio de Milnor para $f$ em 0, então para cada $\varepsilon \operatorname{com} 0<\varepsilon<\varepsilon_{0}$ existe $\delta_{\varepsilon}>0$ tal que

$$
f: \partial B_{\varepsilon} \cap f^{-1}\left(\stackrel{\circ}{B}_{\delta_{\varepsilon}} \backslash\{0\}\right) \rightarrow \stackrel{\circ}{B}_{\delta_{\varepsilon}} \backslash\{0\}
$$

é uma submersão própria.

Demonstração. Basta tomar $\varepsilon^{\prime}$ tal que $0<\varepsilon^{\prime}<\varepsilon$ e aplicar a Proposição 4.1.2.

Teorema 4.1.1. Suponha que $f$ satisfaz as condições (a) e (b) de Milnor em 0, e seja $\varepsilon_{0}$ um raio de Milnor para $f$ em 0 . Então, para todo $\varepsilon>0$, com $0<\varepsilon<\varepsilon_{0}$, existe $\delta_{\varepsilon}>0$ tal que a aplicação

$$
f: B_{\varepsilon} \cap f^{-1}\left(B_{\delta_{\varepsilon}} \backslash\{0\}\right) \rightarrow B_{\delta_{\varepsilon}} \backslash\{0\}
$$

é uma fibração suave localmente trivial.

Demonstração. Seja $B_{\varepsilon} \subset \mathbb{R}^{n}$ a bola fechada com centro na origem e raio $\varepsilon$, onde $\varepsilon$ é um raio de Milnor para $f$ em 0 . Para este $\varepsilon$, considere $\delta_{\varepsilon}$ suficientemente pequeno tal que

$$
f: S_{\varepsilon}^{n-1} \cap f^{-1}\left(B_{\delta_{\varepsilon}} \backslash\{0\}\right) \rightarrow B_{\delta_{\varepsilon}} \backslash\{0\}
$$

é uma submersão suave sobrejetora. Isto é possível pela condição de Milnor (b). 
Pela condição (a) de Milnor, temos que a aplicação

$$
f: \stackrel{\circ}{B}_{\varepsilon} \cap f^{-1}\left(B_{\delta \varepsilon} \backslash\{0\}\right) \rightarrow B_{\delta_{\varepsilon}} \backslash\{0\}
$$

também é uma submersão suave sobrejetora.

Segue então do Teorema de Ehresmann que a aplicação

$$
f: B_{\varepsilon} \cap f^{-1}\left(B_{\delta \varepsilon} \backslash\{0\}\right) \rightarrow B_{\delta_{\varepsilon}} \backslash\{0\}
$$

é fibração suave localmente trivial.

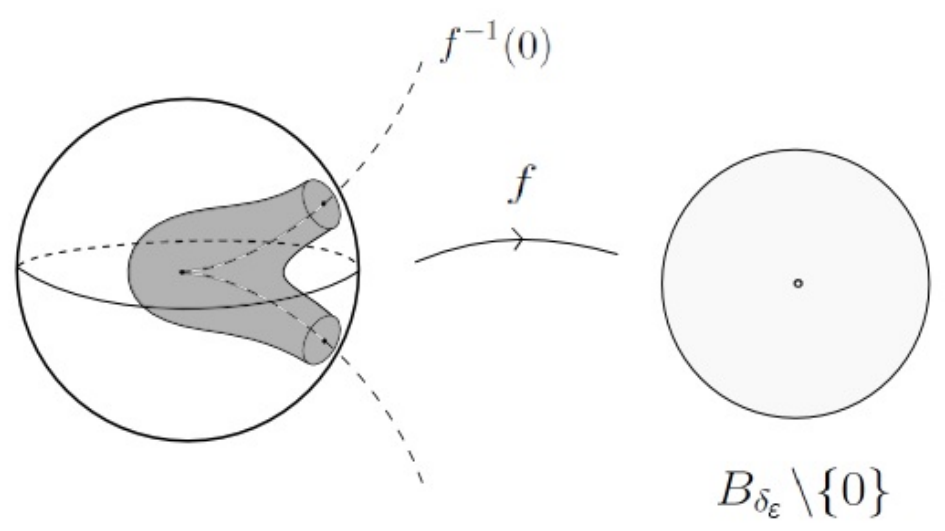

Corolário 4.1.2. Suponha que $f$ satisfaz as condições (a) e (b) de Milnor em 0, e seja $\varepsilon_{0}$ um raio de Milnor para $f$ em 0 .

Então, para todo $\varepsilon$ tal que $0<\varepsilon<\varepsilon_{0}$, existe $\delta_{\varepsilon}>0$ tal que a aplicação

$$
f: B_{\varepsilon} \cap f^{-1}\left(\stackrel{\circ}{B}_{\delta_{\varepsilon}} \backslash\{0\}\right) \rightarrow \stackrel{\circ}{B}_{\delta_{\varepsilon}} \backslash\{0\}
$$

é uma submersão (estratificada) própria e, logo, uma fibração localmente trivial.

Além disso, para todo tal par $\left(\varepsilon, \delta_{\varepsilon}\right)$, para todo $\delta$ tal que $0<\delta<\delta_{\varepsilon}$, a aplicaçãa

$$
f: B_{\varepsilon} \cap f^{-1}\left(\partial B_{\delta}\right) \rightarrow \partial B_{\delta}
$$

é uma submersão (estratificada) própria e, logo, uma fibração localmente trivial. 
Segue que, para todo tal par $\left(\varepsilon, \delta_{\varepsilon}\right)$, para todo $\delta$ tal que $0<\delta<\delta_{\varepsilon}$, a aplicação

$$
f: \stackrel{\circ}{B}_{\varepsilon} \cap f^{-1}\left(\partial B_{\delta}\right) \rightarrow \partial B_{\delta}
$$

é uma fibração suave localmente trivial.

Corolário 4.1.3. Suponha que $f$ satisfaz as condições (a) e (b) de Milnor em 0, e que $k>$ 1. Então, f leva uma vizinhança aberta da origem sobrejetivamente em uma vizinhança aberta da origem.

\subsection{Condições de Milnor para funções holomorfas}

Seja $f:\left(\mathbb{C}^{n}, 0\right) \rightarrow(\mathbb{C}, 0)$ um germe de função holomorfa. Podemos considerar $f$ como um germe de aplicação de $\mathbb{R}^{2 n}$ em $\mathbb{R}^{2}$. Levantamos a seguinte questão:

$$
\text { Será que } f \text { satisfaz as condições de Milnor (a) e (b) na origem? }
$$

Dedicamos esta seção à resposta afirmativa desta pergunta através das ferramentas da teoria de estratificação. Assim, vamos introduzir os conceitos e resultados necessários para o desenvolvimento do nosso estudo.

Definição 4.2.1. Dizemos que uma partição $\left(V_{i}\right)_{i \in I}$ de um conjunto analítico $V \subset U \subset$ $\mathbb{C}^{n}$ é uma estratificação se a partição $\left(V_{i}\right)_{i \in I}$ verifica as seguintes propriedades:

- cada $V_{i}$ é uma subvariedade analítica conexa de $U$;

- para cada $V_{i}$ temos que $\overline{V_{i}}$ e $\overline{V_{i}}-V_{i}$ são conjuntos analíticos. Neste caso, $V_{i}$ é dito $U$-estrito;

- a partição $\left(V_{i}\right)_{i \in I}$ é localmente finita, isto é, para todo ponto $p \in V$ existe uma vizinhança de $W_{p}$ de $p$ em $V$ tal que, $W_{p} \cap V_{j} \neq \varnothing$, somente para um número finito de indices $j$;

- a partição $\left(V_{i}\right)_{i \in I}$ satisfaz a condição de fronteira, isto é, se $V_{i} \cap \bar{V}_{j} \neq \varnothing$, onde $\bar{V}_{j}$ é o fecho de $V_{j}$ em $U$, então $V_{i} \subset \bar{V}_{j}$. 
Definição 4.2.2. Seja $V \subset U \subset \mathbb{C}^{n}$ um conjunto analítico. Seja $M \subset V$ uma subvariedade suave de $U$ contida em $V$. Dizemos que $V$ satisfaz a condição (a) de Whitney ao longo de $M$ se, para toda sequência de pontos $\left(x_{k}\right)$ na parte regular $V \backslash \Sigma(V)$ de $V$ que converge para o ponto $x$ de $M$ e a correspondente sequência de planos tangentes $\left(T_{x_{k}} V\right)$ converge para um espaço vetorial $T$ na variedade de Grasmann correspondente, então o espaço $T$ contém $T_{x} M$.

Definição 4.2.3. Dizemos que uma estratificação $\left(V_{i}\right)_{i \in I}$ do conjunto analítico $V \subset U \subset$ $\mathbb{C}^{n}$ satisfaz a condição (a) de Whitney se, para todo par de estratos $V_{i}, V_{j}$ tal que $i \neq j$ e $V_{i} \subset \bar{V}_{j}$, o conjunto analítico $\bar{V}_{j} \subset U \subset \mathbb{C}^{n}$ satisfaz a condição (a) de Whitney ao longo de $V_{i}$.

Em [48] Whitney provou o seguinte resultado geral.

Teorema 4.2.1. Todo conjunto analítico admite uma estratificação que satisfaz a condição (a) de Whitney.

Teorema 4.2.2. Seja $V \subset U \subset \mathbb{C}^{n}$ um conjunto analítico. Suponha que $W$ é um aberto da parte regular $V \backslash \Sigma(V)$ de $V$ que é $U$-estrito e tal que $\bar{W}=V$. Então existe uma estratificação $\Gamma=\left(V_{i}\right)_{i \in I}$ de $V$ que satisfaz a condição (a) de Whitney tal que $V \backslash W$ é uma união de estratos e que as componentes conexas de $W$ são estratos de $\Gamma$.

A seguir vamos definir o que chamamos de boa estratificação de um conjunto analítico. Consideramos $0 \in U \subseteq \mathbb{C}^{n}$ subconjunto aberto e $f: U \rightarrow \mathbb{C}$ função holomorfa. Denotaremos por $H_{0}$ a hipersuperfície $\{x \in U ; f(x)=0\}$ e para $x \in U$ fixo por $H_{f(x)}$ a hipersuperfície $\{y \in U ; f(y)=f(x)\}$ que passa por $x$. Também denotaremos por $\Gamma=\left(V_{i}\right)_{i \in I}$ uma estratificação de $H_{0}$.

Definição 4.2.4. Dizemos que $\Gamma$ é uma boa estratificação de $H_{0}$ em 0 , se existe uma vizinhança aberta $\Omega$ de 0 em $\mathbb{C}^{n}$ tal que, para toda sequência de pontos $\left(x_{k}\right)$ de $\Omega \backslash H_{0}$ que converge para um ponto $x$ de $H_{0} \cap \Omega$ e cuja sequência de planos tangentes correspondentes $T_{x_{k}} H_{f\left(x_{k}\right)}$ a $H_{f\left(x_{k}\right)}$ em $x_{k}$ é definida e converge para um hiperplano $T$, o hiperplano $T$ contém o plano tangente em $x$ ao estrato de $\Gamma$ que contém $x$. Dizemos também que $\Gamma$ é uma boa estratificação de $H_{0}$ em $\Omega$. 
O principal resultado desta seção mostra que dada $f$ como acima numa vizinhança da origem, a hipersuperfície $H_{0}$ sempre admite uma boa estratificação. Para isso, precisamos de alguns preliminares.

Definição 4.2.5. Seja $f: U \rightarrow \mathbb{C}$ função holomorfa, com $0 \in U, f(0)=0$. Diremos que $f$ satisfaz a desigualdade de Lojasiewicz na origem se, existe uma vizinhança $W$ de 0 contida em $U$, tal que para todo $z \in W$ temos:

$$
\|\nabla f(z)\| \geq c|f(z)|^{\theta}, \quad \operatorname{com} 0<\theta<1 \text { e } c>0 .
$$

Teorema 4.2.3. [23], [5]. Toda função holomorfa definida numa vizinhança da origem, satisfaz a desigualdade de Eojasewicz.

Vamos apresentar abaixo uma prova que toda função holomorfa admite uma boa estratificação. A principal referência utilizada foi [17].

Teorema 4.2.4. (Hamm-Lê, [17]) Toda função holomorfa admite uma boa estratificação.

Demonstração. Seja $f: U \subset \mathbb{C}^{n} \rightarrow \mathbb{C}$ uma função holomorfa. Dado $N \in \mathbb{N}$, defina a função analítica $g: U \times \mathbb{C} \rightarrow \mathbb{C}, g(x, t)=f(x)-t^{N}$ e o conjunto analítico

$$
G=\left\{(x, t) \in U \times \mathbb{C} ; f(x)-t^{N}=0\right\} \subset U \times \mathbb{C} .
$$

A seguir vamos encontrar o conjunto singular da variedade analítica $G$.

Observe que, $\nabla g(x, t)=\left(\nabla f(x),-N t^{N-1}\right)$. Logo, temos que $\nabla g(x, t)=(0,0)$ se e somente se, $\nabla f(x)=0$ e $t=0$. Portanto, o conjunto $\Sigma(g)=\Sigma(f) \times\{0\}$ e que claramente satisfaz a inclusão

$$
\Sigma(g) \subseteq H_{0} \times\{0\}
$$

Pelo Teorema 4.2.2 podemos considerar uma estratificação $\Gamma_{N}$ de $G$ satisfazendo a condição de Whitney $(a)$, tal que $G \backslash H_{0} \times\{0\}$ e $H_{0} \times\{0\}$ é uma união de estratos. Essa estratificação induz uma estratificação $S_{N}$ de $H_{0}$ também satisfazendo a condição de Whitney $(a)$. 
Nosso objetivo é mostrar que para $N$ suficientemente grande $S_{N}$ é uma boa estratificação de $H_{0}$ em alguma vizinhança da origem $0 \in U$. Para isso, mostraremos que $\Gamma_{N}$ é uma boa estratificação de $G$ na origem, para alguma vizinhança de $\Omega$ da origem $U \times \mathbb{C}$.

Seja $x_{n}=\left(u_{n}, t_{n}\right)$ uma sequência em $G \backslash H_{0} \times\{0\}$, em alguma bola compacta $B$ com centro na origem contida em $U \times \mathbb{C}$, com $x_{n} \rightarrow(x, 0) \in\left(H_{0} \times\{0\}\right) \cap B$.

A menos de subsequência, podemos assumir que a sequência de planos tangentes $T_{x_{n}}\left(H_{f\left(u_{n}\right)} \times t_{n}\right)$ converge para um limite $T$. Basta mostrar que $T \supset T_{x} V_{x}$, onde $V_{x}$ é algum estrato de $H_{0}$ contendo o ponto $x$.

Observe que como $x_{n} \in G \backslash \Sigma(g)$, temos que o plano tangente $T_{x_{n}} G$ está bem definido e para cada $t_{n}$ fixo, o conjunto analítico $H_{f\left(u_{n}\right)} \times\left\{t_{n}\right\}=\left\{\left(x, t_{n}\right): f(x)=t_{n}^{N}\right\}$ é uma subvariedade analítica de $G$. Logo, temos que $T_{\left(x_{n}\right)}\left(H_{f\left(u_{n}\right)} \times\left\{t_{n}\right\}\right)$ é um subespaço vetorial complexo de codimensão 1 em $T_{x_{n}} G$.

Suponha que

$$
T_{x} V_{x} \not T
$$

A menos de subsequência podemos supor que $T_{x_{n}} G$ converge para $\tau$. Como $\Gamma_{N}$ satisfaz a condição de Whitney $(a)$, temos que $T_{x} V_{x} \subset \tau$. Da inclusão $T_{x_{n}}\left(H_{f\left(u_{n}\right)} \times\left\{t_{n}\right\}\right) \subset T_{x_{n}} G$ obtemos que $T \subset \tau$. Observe que a dimensão complexa de $\tau$ é $n$ e a de $T$ é $n-1, \operatorname{logo}$ por (4.2.1) obtemos que

$$
\tau=T+T_{x} V_{x}
$$

Por outro lado, $T \subset \mathbb{C}^{n} \times\{0\}$ e $T_{x} V_{x} \subset \mathbb{C}^{n} \times\{0\}$, por (4.2.2) temos que

$$
\tau=\mathbb{C}^{n} \times\{0\} .
$$

Para analisar o limite do plano tangente $T_{x_{n}} G$, estudaremos o comportamento do vetor complexo normal

$$
\frac{\nabla g\left(x_{n}\right)}{\left\|\nabla g\left(x_{n}\right)\right\|} .
$$

Observe que

$$
\frac{\nabla g\left(x_{n}\right)}{\left\|\nabla g\left(x_{n}\right)\right\|}=\frac{\left.\left(\nabla f\left(u_{n}\right),-N t_{n}^{N-1}\right)\right)}{\sqrt{\left\|\nabla f\left(u_{n}\right)\right\|^{2}+\left|N t_{n}^{N-1}\right|^{2}}}=\left(\frac{\nabla f\left(u_{n}\right)}{\left\|\nabla f\left(u_{n}\right)\right\|}, \frac{-N t_{n}^{N-1}}{\left\|\nabla f\left(u_{n}\right)\right\|}\right) \cdot \frac{1}{\sqrt{1+\frac{\left|N t_{n}^{N-1}\right|^{2}}{\left\|\nabla f\left(u_{n}\right)\right\|^{2}}}} .
$$


Vamos analisar agora a seguinte expressão:

$$
\frac{N\left|t_{n}\right|^{N-1}}{\left\|\nabla f\left(u_{n}\right)\right\|} .
$$

Observe que, como $x_{n}=\left(u_{n}, t_{n}\right) \in G$, temos que $f\left(u_{n}\right)=t_{n}^{N}$, logo obtemos $\left|t_{n}\right|^{N-1}=$ $\left|f\left(u_{n}\right)\right|^{\frac{N-1}{N}}$ e por (4.2.4) obtemos

$$
\frac{N\left|f\left(u_{n}\right)\right|^{\frac{N-1}{N}}}{\left\|\nabla f\left(u_{n}\right)\right\|} .
$$

Pela desigualdade de Łojasiewicz sabemos que $\left\|\nabla f\left(u_{n}\right)\right\| \geq c\left|f\left(u_{n}\right)\right|^{\theta}$, logo temos $\left|t_{n}\right|^{N-1}=$ $\left|f\left(u_{n}\right)\right|^{\frac{N-1}{N}}$, obtendo a desigualdade

$$
\frac{N\left|t_{n}\right|^{N-1}}{\left\|\nabla f\left(u_{n}\right)\right\|} \leq\left|f\left(u_{n}\right)\right|^{\frac{N-1}{N}-\theta}
$$

Observe que escolher um natural $N$ tal que $N>\frac{1}{1-\theta}$ é equivalente a $\frac{N-1}{N}-\theta>0$. Logo escolhendo tal $N$, como $x_{n}=\left(u_{n}, t_{n}\right) \rightarrow(x, 0) \in H_{0} \times\{0\}$ temos que $f\left(u_{n}\right) \rightarrow f(x)=0$. Portanto, por (4.2.5) acima temos $\frac{N\left|t_{n}\right|^{N-1}}{\left\|\nabla f\left(u_{n}\right)\right\|} \rightarrow 0$, quando $n \rightarrow+\infty \mathrm{e}$

$$
\frac{\nabla g\left(x_{n}\right)}{\left\|\nabla g\left(x_{n}\right)\right\|} \rightarrow\left(z_{0}, 0\right), z_{0} \in \mathbb{C}^{n} \backslash\{0\}
$$

Mas como $\left(z_{0}, 0\right)$ é o vetor normal de $\tau$, obtemos por (4.2.3) uma contradição.

Por um argumento análogo ao da prova do Lema 3.1.1 é possível provar que dado um conjunto analítico $V, p \in V$, existe uma estratifição $(a)$ de Whitney $\Gamma$ de $V$, tal que para todo $\varepsilon>0$ suficientemente pequeno, os estratos que contêm o ponto $p$ no seu fecho são transversais a toda esfera $S_{\varepsilon}(p)$ centrada no ponto $p$ e raio $\varepsilon$.

Corolário 4.2.1. Dada $f$ holomorfa numa vizinhança da origem, existe $\varepsilon>0$ suficientemente pequeno tal que, todas as fibras "vizinhas" à fibra $H_{0}$ são transversais a $S_{\varepsilon}$.

Corolário 4.2.2. Toda função holomorfa $f$ numa vizinhança da origem admite um raio de Milnor (b) na origem. 


\subsection{Topologia da fibra de Milnor real}

O principal objetivo dessa seção é provar um resultado geral devido a J. Milnor ([27]) sobre o grau de conexidade da fibra de um germe polinomial $f:\left(\mathbb{R}^{n}, 0\right) \rightarrow\left(\mathbb{R}^{k}, 0\right)$ com singularidade isolada na origem. Esse resultado decorre de parte da demonstração do Lema 11.4, página 100 de [27].

Proposição 4.3.1. Seja $f:\left(\mathbb{R}^{n}, 0\right) \rightarrow\left(\mathbb{R}^{k}, 0\right)$ um germe de aplicação polinomial com $\Sigma(f)=\{0\}$. Se o link $K$ não for vazio, então a fibra da fibração de Milnor é $(k-2)-$ conexa.

Para vermos uma ideia da prova da Proposição precisamos desenvolver os seguintes resultados.

Dada $f: U \subset \mathbb{R}^{n} \rightarrow \mathbb{R}^{k}$, com $f(0)=0$ com acima, pela Proposição 4.1.1, sabemos que $f$ admite um raio de Milnor, digamos $\varepsilon_{0}$. Ou seja, para todo $\varepsilon>0$ com $0<\varepsilon \leq \varepsilon_{0}$, $0 \in \mathbb{R}^{k}$ é valor regular da aplicação restrição

$$
f: S_{\varepsilon}^{n-1} \rightarrow \mathbb{R}^{k}
$$

Sendo o link $K$ não vazio, sabemos que para cada $\varepsilon$ existe $\eta=\eta(\varepsilon), 0<\eta \ll \varepsilon$ e um disco $k$-dimensional fechado $D_{\eta} \subset \mathbb{R}^{k}$ centrado na origem tal que a aplicação

$$
f: S_{\varepsilon} \cap f^{-1}\left(D_{\eta}\right) \rightarrow D_{\eta}
$$

é uma submersão suave e própria. Logo, pelo Teorema de Ehresmann temos uma fibração suave trivial, visto que a base é contrátil.

Sendo a fibra o link $K$, temos o seguinte difeomorfismo:

$$
S_{\varepsilon} \cap f^{-1}\left(D_{\eta}\right) \approx K \times D_{\eta}
$$

Vamos denotar por $T=S_{\varepsilon} \cap f^{-1}\left(D_{\eta}\right)$ a vizinhança tubular fechada do link $K$ em $S_{\varepsilon}$. 
Ainda para esses $\varepsilon$ e $\eta$ temos do Corolário 4.1.2 que a aplicação

$$
f: B_{\varepsilon} \cap f^{-1}\left(S_{\eta}^{k-1}\right) \rightarrow S_{\eta}^{k-1}
$$

é uma fibração suave localmente trivial.

Por [27], página 99, Lemma 11.3, temos que existe um campo de vetores $v(x)$ em $B_{\varepsilon} \backslash V$ tal que:

1. $\langle v(x), x\rangle>0$

2. $\left\langle v(x), \nabla\|f(x)\|^{2}\right\rangle>0$

A condição (1) significa que o fluxo do campo é transversal a todas as pequenas esferas centradas na origem. E a condição (2) significa que o fluxo do campo aponta na direção em que a função é crescente. Esse campo pode ser obtido a partir da bissetriz dos campos $\nabla\|x\|^{2}$ e $\nabla\|f(x)\|^{2}$.

Milnor mostrou no Lema acima que o fluxo deste campo define um difeomorfismo da variedade com bordo $B_{\varepsilon} \cap f^{-1}\left(S_{\eta}^{k-1}\right)$ para a variedade com bordo $S_{\varepsilon} \backslash \operatorname{int}(T)$, onde $\operatorname{int}(T)$ é o interior da vizinhança tubular $T$, que restrito ao bordo $\partial(T)=S_{\varepsilon}^{n-1} \cap f^{-1}\left(S_{\eta}^{k-1}\right)$ é a aplicação identidade.

Agora considere uma decomposição celular do link $K=\cup_{i=1}^{s} C_{i}$. Pelo difeomorfismo (4.3.1) podemos obter a esfera $S_{\varepsilon}$ de $S_{\varepsilon} \backslash \operatorname{int}(T)$ adjuntando um número de células de dimensão $\geq k$, uma $(k+i)$-célula para cada $i$-célula de $K$.

Portanto, segue o seguinte isomorfismo:

$$
\pi_{i}\left(S_{\varepsilon} \backslash \operatorname{int}(T)\right)=\pi_{i}\left(S_{\varepsilon}\right)=0
$$

para todo $0 \leq i \leq k-2$. A última igualdade pode ser encontrada, por exemplo em [18], p. 349, Corolário 4.9 .

Com isto demonstramos a seguinte Proposição.

Proposição 4.3.2. Seja $f: U \subseteq \mathbb{R}^{n} \rightarrow \mathbb{R}^{k}$ um germe de aplicação polinomial, com $f(0)=0$ e $\Sigma(f)=\{0\}$. Então, a variedade $B_{\varepsilon} \cap f^{-1}\left(S_{\eta}^{k-1}\right)$ do espaço fibrado (4.3.2) é 
$(k-2)$-conexo.

Agora, de (4.3.1) temos que o espaço $T \approx K \times D_{\eta}$, logo $\partial(T) \approx K \times S_{\eta}^{k-1}$ e claramente temos os mergulhos $S_{\eta}^{k-1} \hookrightarrow \partial(T) \hookrightarrow S_{\varepsilon} \backslash \operatorname{int}(T)$. Disto segue que a sequência exata

$$
0 \rightarrow \pi_{i}(F) \rightarrow \pi_{i}\left(S_{\varepsilon} \backslash \operatorname{int}(T)\right) \rightarrow \pi_{i}\left(S_{\eta}^{k-1}\right) \rightarrow 0
$$

cinde; ou seja, temos o seguinte isomorfismo:

$$
\pi_{i}\left(S_{\varepsilon} \backslash \operatorname{int}(T)\right)=\pi_{i}(F) \oplus \pi_{i}\left(S_{\eta}^{k-1}\right) .
$$

Como já provamos acima que $\pi_{i}\left(S_{\varepsilon} \backslash \operatorname{int}(T)\right)=0=\pi_{i}\left(S_{\eta}^{k-1}\right)$, para todo $0 \leq i \leq k-2$, segue diretamente que a fibra $F$ é $(k-2)$-conexa e a Proposição 4.3 .1 está provada.

De modo análogo ao que foi feito no caso de germe de função holomorfa, mais recentemente os autores de [3] provaram a seguinte fórmula para a caracterítica de Euler da fibra real.

Proposição 4.3.3. Sejam $f:\left(\mathbb{R}^{n}, 0\right) \rightarrow\left(\mathbb{R}^{p}, 0\right), n \geq p \geq 2$, um germe de aplicação polinomial com $\Sigma(f)=\{0\}$ e denote por $F_{f}$ a fibra da fibração de Milnor. Então:

1. Se n é par, então $\chi\left(F_{f}\right)=1-\operatorname{deg}_{0}\left(\nabla f_{1}\right)$. Além disso, $\operatorname{deg}_{0}\left(\nabla f_{1}\right)=\cdots=\operatorname{deg}_{0}\left(\nabla f_{p}\right)$.

2. se n é impar, então $\chi\left(F_{f}\right)=1$. Além disso, $\operatorname{deg}_{0}\left(\nabla f_{i}\right)=0$, para todo $i=1, \cdots, p$. 


\section{Capítulo}

\section{5}

\section{Singularidades no Infinito}

\subsection{Estudo global da singularidade}

O estudo da fibração global para funções polinomiais complexas em espaço afim tem por objetivo encontrar condições de regularidades sobre as quais um polinômio complexo apresente um "bom" comportamento no infinito. Isto significa que a fibra afim não tem a topologia "afetada" por singularidades que "moram" no infinito. Para ilustrar melhor a situação vamos verificar um exemplo.

Exemplo 5.1.1. Considere o polinômio $f: \mathbb{C}^{2} \rightarrow \mathbb{C}, f(x, y)=x-x^{2} y$. É fácil verificar que o conjunto singular $\Sigma(f)$ é vazio. Resolvendo a equação $f(x, y)=0$ obtemos

$$
\left\{(x, y) \in \mathbb{C}^{2} ; x=0\right\} \sqcup\left\{(x, y) \in \mathbb{C}^{2} ; x \in \mathbb{C}^{*}, y=\frac{1}{x}\right\}
$$

Logo, a fibra nula tem duas componentes conexas e é, a menos de difeomorfismo, dada por $\mathbb{C} \sqcup \mathbb{C}^{*}$. Por outro lado, para qualquer $c \in \mathbb{C}^{*}$, quando resolvemos a equação $f(x, y)=c$ obtemos

$$
\left\{(x, y) \in \mathbb{C}^{2} ; x \in \mathbb{C}^{*}, y=\frac{x-c}{x^{2}}\right\}
$$

Portanto, parametrizando o gráfico temos que a fibra é difeomorfa a $\mathbb{C}^{*}$.

Sendo a aplicação regular (ou seja, sem pontos críticos em $\mathbb{C}^{2}$ ), como podemos explicar 
a mudança no comportamento topológico da fibra?

Neste caso fica clara a necessidade de um critério matemático que possa detectar esses tipos de mudança no comportamento, pois o senso comum diz que em havendo mudança no tipo topológico, espera-se passar por uma singularidade.

Nesta situação, atribuimos a mudança topológica à "existência de singularidades no infinito".

Em [16] Hà e Lê provaram um critério para detectar os valores atípicos para funções complexas em duas variáveis. O principal resultado foi:

Teorema 5.1.1. Sejam $f: \mathbb{C}^{2} \rightarrow \mathbb{C}$ uma função polinomial e $z \in \mathbb{C}$. Então, $f$ é uma fibração suave localmente trivial numa vizinhança de z (isto é, existe $U_{z}$ vizinhança de z em $\mathbb{C}$, tal que a aplicação $f: \mathbb{C}^{2} \cap f^{-1}\left(U_{z}\right) \rightarrow U_{z}$ é uma fibração local suave) se, e somente se, z é um valor regular e a característica de Euler-Poincaré $\chi\left(f^{-1}(y)\right)=\chi\left(f^{-1}(z)\right)$ para todo $y \in U_{z}$.

Um critério deste tipo se fazia necessário pois, como vimos no exemplo, as fibras de um polinômio no ambiente afim podem não ter o mesmo tipo topológico globalmente ainda que sem a presença de singularidades. No exemplo acima vimos que, embora $0 \in \mathbb{C}$ seja um valor regular de $f$, claramente $\chi\left(f^{-1}(c)\right) \neq \chi\left(f^{-1}(0)\right)$, para todo $c \neq 0$, logo podemos concluír que $\{0\}$ é o único valor atípico.

A seguir apresentamos algumas definições e resultados básicos sobre condições de regularidade no infinito e verificamos como a chamada "fibração de Milnor global" pode ser estudada neste contexto.

\subsection{Fibração global}

É conhecido que, dado um polinômio holomorfo de grau $d, f: \mathbb{C}^{n} \rightarrow \mathbb{C}, \operatorname{com} f(z)=$ $f_{1}(z)+\ldots+f_{d}(z)$ em que $f_{i}(z)$ denota a parte homogênea de grau $i$, existe um subconjunto finito $\Gamma \subset \mathbb{C}$ tal que a aplicação

$$
f: \mathbb{C}^{n} \backslash f^{-1}(\Gamma) \rightarrow \mathbb{C} \backslash \Gamma
$$


é uma fibração suave localmente trivial.

O menor tal subconjunto $\Gamma$, no sentido da inclusão, que torna tal aplicação acima uma fibração suave, é conhecido com conjunto dos valores atipicos e denotado por $B_{f}$.

De forma geral, é conhecida a seguinte inclusão: $f(\Sigma(f)) \subset B_{f}$. Além dos valores críticos, sabe-se que este conjunto também contém os chamados "valores críticos no infinito" provenientes do comportamento assintótico da fibra no infinito (singularidade no infinito). Daí, podemos concluir que no exemplo acima temos: $\{0\} \subset B_{f}$.

Observe que, sendo o espaço $\mathbb{C} \backslash B_{f}$ conexo, todas as fibras são difeomorfas e elas são chamadas de fibras genéricas. Além do mais, é também conhecido que essas fibras têm o tipo de homotopia de um CW complexo de dimensão $n-1$.

No que segue apresentamos algumas técnicas e ferramentas que nos ajudarão a detectar tais singularidades no infinito.

Considere o sistema de coordenadas $z=\left(z_{1}, \ldots, z_{n}\right)$ e $f$ polinomial de grau $d$, como acima. Queremos estudar o comportamento topológico no "infinito" da fibra $f(z)=t$, para $t \in \mathbb{C}$. Para isso, podemos estudar o conjunto algébrico $\widehat{f}(z, t)=f(z)-t=$ 0. Como estamos interessados no comportamento no infinito, então precisamos passar para um espaço adequado que contenha as informações das fibras no infinito. Assim, vamos homogeneizar o polinômio $\widehat{f}$ usando uma variável auxiliar, digamos $z_{0}$, e obtermos o polinômio $F\left(z, z_{0}, t\right)=f_{1}(z) z_{0}^{d-1}+f_{2}(z) z_{0}^{d-2}+\ldots+f_{d-1}(z) z_{0}+f_{d}(z)-t z_{0}^{d}$. Como $F(z, 1, t)=\widehat{f}$, então estudar o comportamento do conjunto $\widehat{f}$ no infinito é equivalente a estudar o conjunto algébrico $F=0$ em $P^{n}(\mathbb{C})$.

Defina o seguinte conjunto:

$$
\mathbb{X}=\left\{\left(\left(z: z_{0}\right), t\right) \in P^{n}(\mathbb{C}) \times \mathbb{C} ; F\left(z, z_{0}, t\right)=0\right\}
$$

Este espaço contém todas as informações das fibras no espaço afim e no infinito. Vamos encontrar agora os pontos singulares do conjunto algébrico $\mathbb{X}$, o que significa encontrar a solução da equação

$$
\nabla F\left(z, z_{0}, t\right)=0
$$


Temos:

$$
\begin{gathered}
\frac{\partial F}{\partial z_{i}}\left(z, z_{0}, t\right)=\sum_{j=1}^{d} \frac{\partial f_{j}}{\partial z_{i}}(z) z_{0}^{d-j}=0 \text { para } i=1, \ldots, n \\
\frac{\partial F}{\partial z_{0}}\left(z, z_{0}, t\right)=\sum_{j=1}^{d}(d-j) f_{j}(z) z_{0}^{d-j-1}=0 ; \\
\frac{\partial F}{\partial t}\left(z, z_{0}, t\right)=z_{0}^{d}=0 .
\end{gathered}
$$

Do sistema acima concluímos que o conjunto singular de $\mathbb{X}$ no infinito é dado por

$$
\Sigma_{f}^{\infty}=\left\{\left(z_{1}: \ldots: z_{n}: 0\right) \in P^{n}(\mathbb{C}) ; \nabla f_{d}(z)=0=f_{d-1}(z)\right\}
$$

Vamos encontrar este conjunto no exemplo anterior.

Exemplo 5.2.1. Considerando $f=x-x^{2} y$ temos $f_{3}=-x^{2} y$ e $f_{2} \equiv 0$. Logo, $\nabla f_{3}(x, y)=$ $\left(-2 x y, x^{2}\right)=(0,0)$, tem como solução $x=0$. Segue que $\Sigma_{f}^{\infty}=\left\{(0: 1: 0) \in P^{2}(\mathbb{C})\right\}$ e, sendo $(0,1) \in \mathbb{C}^{2}$, obtemos que $f(0,1)=0$ e portanto $0 \in \mathbb{C}$ é o único valor atipico $e$ $B_{f}=\{0\}$.

Isto motiva a seguinte definição.

Definição 5.2.1. Seja $f: \mathbb{C}^{n} \rightarrow \mathbb{C}$, uma função polinomial. Diremos que $c \in \mathbb{C}$ é um valor regular no infinito, se existir um disco $D \subset \mathbb{C}$ centrado em c e um subconjunto compacto $K \subset \mathbb{C}^{n}$ tal que, $f: f^{-1}(D) \backslash K \rightarrow D$ é uma fibração suave localmente trivial. Caso contrário, diremos que c é um valor crítico no infinito.

Segue do exposto acima que $c=0$ é um valor crítico no infinito da função $f=x-x^{2} y$. Definição 5.2.2. Seja $f: \mathbb{C}^{n} \rightarrow \mathbb{C}$ uma função polinomial. Diremos que $f$ admite uma fibração de Milnor no infinito (ou fibração de Milnor global) se existir um $R_{0}>0$ suficientemente grande, tal que para todo $R \geq R_{0}$ a aplicação

$$
\frac{f}{\|f\|}: S_{R}^{2 n-1} \backslash K^{\infty} \rightarrow S^{1}
$$

for uma fibração suave localmente trivial, onde $K^{\infty}:=f^{-1}(0) \cap S_{R}^{2 n-1}$ é chamado o link de $f$ no infinito. 
Para o caso $n=2$, foi mostrado por Arnaud Bodin em [6] que é possível utilizar a existência da fibração de Milnor global para detectar os valores críticos no infinito. No que segue apresentamos seu principal resultado.

Teorema 5.2.1. Seja $f: \mathbb{C}^{2} \rightarrow \mathbb{C}$ polinomial. Então, $c \neq 0$ é valor regular no infinito se, e somente se, $f$ admite fibração de Milnor no infinito.

A prova deste resultado usa técnicas de resolução da singularidade e outras ferramentas que fogem do escopo deste trabalho.

Ainda no caso $n=2$, existem outras caracterizações dos valores atípicos no infinito. Veja, por exemplo, $[12,16,33,47]$, no entanto, no caso geral, para $f: \mathbb{C}^{n} \rightarrow \mathbb{C}$ polinomial, $n \geq 3$, o problema de caracterizar os valores atípicos ainda é um problema em aberto. Uma abordagem para detectar os valores de $B_{f} \backslash f(\Sigma(f))$ (ou controlar o comportamento assintótico das fibras) é via as chamadas condições de regularidade no infinito. Por exemplo: $\rho_{E}$-regularidade ([46]), t-equisingularidade ([42]), condições de Malgrange $([33,46])$.

Assim, na direção de determinar boas condições de regularidade no infinito, foram introduzidas algumas classes especiais de polinômios. Por exemplo Broughton em [8] introduziu a classe dos polinômios tame como segue:

Definição 5.2.3. Seja $f: \mathbb{C}^{n} \rightarrow \mathbb{C}$ uma função polinomial. Dizemos que $f$ é tame se existem um $\delta>0$ e uma vizinhança compacta $K$ de $\mathbb{C}^{n}$ tal que $\|\nabla d f(x)\| \geq \delta$, para todo $x \in \mathbb{C}^{n} \backslash K$.

Nesta classe foi feita a seguinte caracterização de $B_{f}$.

Teorema 5.2.2. Se $f: \mathbb{C}^{n} \rightarrow \mathbb{C}$ é polinomial tame, então $B_{f}=f(\Sigma(f))$.

Em [30], A. Némethi e Zaharia introduziram também as classes de polinômios quasitame, M-tame e semitame, como a seguir.

Definição 5.2.4. Seja $f: \mathbb{C}^{n} \rightarrow \mathbb{C}$ uma função polinomial, considere

$$
M(f):=\left\{z \in \mathbb{C}^{n} ; \exists \lambda \in \mathbb{C} \operatorname{com} \nabla f(z)=\lambda z\right\},
$$




$$
\Lambda:=\left\{c \in \mathbb{C} ; \exists\left\{z_{k}\right\} \subset \mathbb{C}^{n}, \lim _{k \rightarrow+\infty} \nabla f\left(z_{k}\right)=0 e \lim _{k \rightarrow+\infty}\left(f\left(z_{k}\right)-\left\langle z_{k}, \nabla f\left(z_{k}\right)\right\rangle\right)=c\right\}
$$

$e$

$$
S_{f}:=\left\{c \in \mathbb{C} ; \exists\left\{z_{k}\right\} \subset M(f),\left\|z_{k}\right\| \rightarrow+\infty,\left\|\operatorname{grad} f\left(z_{k}\right)\right\| \rightarrow 0 \text { e } f\left(z_{k}\right) \rightarrow c\right\} .
$$

Nas condições acima temos,

1. Se $\Lambda$ é vazio, diremos que $f$ é quasitame;

2. Se $S_{f}$ for vazio, diremos que $f$ é $M$-tame;

3. $S e S_{f}=\{0\}$, diremos que $f$ é semitame.

De forma geral foi provado em [31] as seguintes inclusões

$$
B_{f} \subseteq f\left(\Sigma_{f}\right) \cup S_{f} \subseteq \Lambda . e S_{f} \subset \Lambda
$$

Em [33] Parusinski considerou as seguintes condições:

Definição 5.2.5. Seja $f: \mathbb{C}^{n} \rightarrow \mathbb{C}$ uma função polinomial e $c \in \mathbb{C}$.

(i) Diremos que a fibra $f^{-1}(c)$ satisfaz a condição de Malgrange, se existir um $\delta>0$ tal que para toda sequência $\left\{z_{k}\right\}$, com $\left\|z_{k}\right\| \rightarrow+\infty$ e $f\left(z_{k}\right) \rightarrow c$, temos que

$$
\left\|z_{k}\right\| \cdot\left\|\nabla f\left(z_{k}\right)\right\| \geq \delta
$$

(ii) Diremos que a fibra $f^{-1}($ c) satisfaz a condição de Malgrange fraca se existirem $N \geq 2$ natural e um $\delta>0$ tal que, para toda sequência $\left\{z_{k}\right\}$, com $\left\|z_{k}\right\| \rightarrow+\infty$ e $f\left(z_{k}\right) \rightarrow c$, temos que

$$
\left\|z_{k}\right\|^{\frac{N}{N-1}} \cdot\left\|\nabla f\left(z_{k}\right)\right\| \geq \delta
$$

É claro que a condição de Malgrange fraca implica a condição de Malgrange. Essa condição é bastante útil para construir campos de vetores numa vizinhança da fibra e garantir que a Definição 5.2.1 é satisfeita.

De forma geral foi provado em [30] que as seguintes implicações são verdadeiras:

$$
\text { tame } \Rightarrow \text { quasitame } \Rightarrow \text { Cond. Malgrange } \Rightarrow M-\text { tame } \Rightarrow \text { semitame }
$$


e exibiu exemplos mostrando que as inclusões são estritas (a menos da primeira, que ainda é um problema em aberto). Ainda em [30] os autores relacionaram a classe semitame com a fibração de Milnor da seguinte forma

Teorema 5.2.3. Se $f$ é semitame, então para $R$ suficientemente grande, a aplicação

$$
\frac{f}{\|f\|}: S_{R}^{2 n-1} \backslash K^{\infty} \rightarrow S^{1}
$$

é a projeção de um fibrado suave localmente trivial.

Esta fibração é conhecida como a fibração de Milnor no infinito. 


\section{Referências Bibliográficas}

[1] R.N. Araújo dos Santos. Topologia de singularidades analíticas. 2011. Tese (Livre Docência em Topologia e Singularidades) - Instituto de Ciências Matemáticas e de Computação, Universidade de São Paulo, São Carlos, 2011. Disponível em: <http://www.teses.usp.br/teses/disponiveis/livredocencia/55/tde$16012012-172219 />$.

[2] R.N. Araújo dos dos Santos. Uniform $(m)$-condition and strong Milnor fibration, Contemporary Mathematics - American Mathematical Society, v. 475, p. 189-198, 2008.

[3] R.N. Araújo dos Santos; D. Dreibelbis; N. Dutertre. Topology of the real Milnor fiber for isolated singularities. Real and complex singularities, Contemp. Math., 569, Amer. Math. Soc., p. 67-75, 2012.

[4] R.N. Araújo dos Santos; M. Tibar. Real map germs and higher open book structures. Geometriae Dedicata, v. 147, p. 177-185, 2010. DOI: 10.1007/s10711009-9449-z

[5] E. Bierstone and P. Milman. Semianalytic and subanalytic sets. Publ. math de I.H.É.S., 67, 5-42, 1988.

[6] A. Bodin. International Mathematics Research Notices, IMRN, 11, p. 615-621, 1999. 
[7] R. Bott and J. Milnor. On the parallelizability of the spheres. Bull. Amer. Soc., 64, p. 87-89, 1958.

[8] S. A. Broughton. On the topology of polynomial hypersurfaces. Proceedings A.M.S. Symp. in Pure. Math., vol 40, I, p. 165-178, 1983.

[9] Y. Chen. Bifurcation values of mixed polynomials and Newton polyhedra. PhD thesis, Université Lille 1, 2012.

[10] A. Dimca. Singularities and topology of hypersurfaces. Springer-Verlag New York, Inc., 1992.

[11] A. Dimca. Topics on Real and Complex Singularities. Friedr Vieweg \& Sohn, 1987.

[12] A. Durfee. Five definitions of critical points at infinity. In Singularities (Oberwolfach, 1996), Progr. Math. 162, p. 345-360, 1998.

[13] T. Gaffney; R.N. Araújo dos Santos. Real integral closure and Milnor fibrations. Real and complex singularities, 146-157, London Math. Soc. Lecture Note Ser., 380, Cambridge Univ. Press, Cambridge, 2010.

[14] A. Garcia, Y. Lequain. Elementos de Álgebra. Instituto de Matemática Pura e Aplicada, 2006.

[15] M. J. Greenberg, J. R. Harper Algebraic Topology: A First Course, Mathematics Lecture Note Series, 1981.

[16] H.V. Hà and D.T. Le. Sur la topologie des polynomes complexes. Acta Math. Vietnam., 9(1), p. 21-32, 1984.

[17] H. A. Hamm and D. T. Lê. Un théorème de Zariski du type de Lefschetz. Ann. Sci. École Norm. Sup. (4) 6 (1973), 317-355.

[18] A. Hatcher. Algebraic Topology. Cambridge University Press, 2002. 
[19] M. Kato and Y. Matsumoto. On the connectivity of the Milnor fiber of a holomorphic function at a critical point. Manifolds-Tokyo 1973 (Proc. Internat. Conf., Tokyo, 1973), p. 131-136, 1975.

[20] E.L. Lima. Grupo fundamental e espaços de recobrimento. Projeto Euclides, IMPA, 2006.

[21] A. Lins Neto. Funções de uma variável complexa. Projeto Euclides, IMPA, 2005.

[22] S. Łojasiewicz. Ensemble semi-analytiques. IHES Lecture notes, 1965.

[23] E.J.N. Looijenga. Isolated Singular Points on Complete Intersections. London Mathematical Society Lecture Note Series, 77. Cambridge University Press, 1984.

[24] D. Massey. Real analytic Milnor fibrations and a strong Eojasiewicz inequality. Real and complex singularities, 268-292, London Math. Soc. Lecture Note Ser., 380, Cambridge Univ. Press, Cambridge, 2010.

[25] A. Menegon. Topology of Varieties with Non-isolated Singularities. PhD thesis, Universidad Nacional Autónoma de México, 2012.

[26] J. Milnor. Morse Theory. Based on lecture notes by M. Spivak and R. Wells. Annals of Mathematics Studies, No. 51. Princeton University Press, Princeton, N.J., 1963.

[27] J. Milnor. Singular points of complex hypersurfaces. Annals of Mathematics Studies. Princeton University Press, 1968.

[28] R. Narasimham. Introduction to the theory of analytic spaces. Lecture Notes in Mathematics, No. 25, Springer-Verlag, Berlin, 1966.

[29] A. Némethi. On the Milnor fiber of non-isolated singularities. Studia Sci. Math. Hungar., vol 43 (1), p. 131-136, 2006. 
[30] A. Némethi and A. Zaharia. Milnor fibration at infinity. Indag. Mathem., 3(3), p. 323-335, 1992.

[31] A. Némethi and A. Zaharia. On the bifurcation set of a polynomial function and newton boundary. Publ. Res. Inst. Math. Sci., 26(4), p. 681-689, 1990.

[32] E. Outerelo, J.M. Ruiz. Mapping Degree Theory. Graduate Studies in Mathematics, Vol. 108. Americam Mathematical Society. 2009.

[33] A. Parusiński. On the bifurcation set of a complex polynomial with isolated singularities at infinity. Compositio Math, 97, p. 369-384, 1995.

[34] L. Paunescu; A. Zaharia, Remarks on the Milnor fibration at infinity. Manuscripta Math. 103 (2000), no. 3, p. 351-361.

[35] G. Prando. O Teorema da Fibração de Milnor e a Topologia das Fibras. Master's thesis, Universidade Federal do Espírito Santo - Centro de Ciências Exatas, 2011.

[36] M.A.S. Ruas and R. Araújo dos Santos. Real Milnor Fibrations and (C)-regularity, Manuscripta Math., 117, no. 2, p. 207-218, 2005.

[37] C. M. Ruiz. Do número de Milnor ao número de Milnor de Lê. Master's thesis, Universidade de São Paulo - Instituto de Ciências Matemáticas e de Computação, 2011.

[38] K. Sakamoto. Milnor fiberings and their characteristic maps. Manifolds-Tokyo 1973 (Proc. Internat. Conf., Tokyo, 1973), p. 145-150, 1974.

[39] H. Sato. Algebraic Topology: An Intuitive Approach. Translations of Mathematical Monographs, vol 183, American Mathematical Society, 1999.

[40] M. Sebastiani. Introdução à Geometria Analítica Complexa. Projeto Euclides, IMPA, 2010.

[41] D. Siersma, A bouquet theorem for the Milnor fiber. J. Algebraic Geom. 4 (1), p. 51-66, 1995. 
[42] D. Siersma and M. Tibăr. Singularities at infinity and their vanishing cycles. Duke Mathematical Journal, 80(3), p. 771-783, 1995.

[43] E. Spanier. Algebraic Topology, McGraw-Hill Book Co., New York, 1966.

[44] N. Steenrod. The Topology of Fibre Bundles. Princeton Mathematical Series, vol. 14. Princeton University Press, Princeton, N.J., 1951.

[45] M. Tibăr Bouquet decomposition of the Milnor fiber, Topology 35 (1), p. 227$241,1996$.

[46] M. Tibăr. Polynomials and Vanishing Cycles, Cambridge University Press, 2007.

[47] M. Tibăr. Regularity at infinity of real and complex polynomial functions. In Singularity theory (Liverpool,1996), London Math. Soc. Lecture Note Ser. 263, p. 249-264, 1999.

[48] H. Whitney. Tangents to analytic varieties, Annal of Math., vol 81, p. 496-549, 1965. 